\title{
Measurement and promotion of physical activity
}

Citation for published version (APA):

Berendsen, B. (2016). Measurement and promotion of physical activity: evaluation of activity monitoirs and a multidisciplinary lifestyle intervention in primary care. [Doctoral Thesis, Maastricht University]. https://doi.org/10.26481/dis.20160624bbb

Document status and date:

Published: 01/01/2016

DOI:

10.26481/dis.20160624bbb

Document Version:

Publisher's PDF, also known as Version of record

\section{Please check the document version of this publication:}

- A submitted manuscript is the version of the article upon submission and before peer-review. There can be important differences between the submitted version and the official published version of record.

People interested in the research are advised to contact the author for the final version of the publication, or visit the DOI to the publisher's website.

- The final author version and the galley proof are versions of the publication after peer review.

- The final published version features the final layout of the paper including the volume, issue and page numbers.

Link to publication

\footnotetext{
General rights rights.

- You may freely distribute the URL identifying the publication in the public portal. please follow below link for the End User Agreement:

www.umlib.nl/taverne-license

Take down policy

If you believe that this document breaches copyright please contact us at:

repository@maastrichtuniversity.nl

providing details and we will investigate your claim.
}

Copyright and moral rights for the publications made accessible in the public portal are retained by the authors and/or other copyright owners and it is a condition of accessing publications that users recognise and abide by the legal requirements associated with these

- Users may download and print one copy of any publication from the public portal for the purpose of private study or research.

- You may not further distribute the material or use it for any profit-making activity or commercial gain

If the publication is distributed under the terms of Article $25 \mathrm{fa}$ of the Dutch Copyright Act, indicated by the "Taverne" license above, 


\section{Measurement and promotion of physical activity}

Evaluation of activity monitors and a multidisciplinary lifestyle intervention in primary care 
The research presented in this thesis was performed at the department of Human Movement Sciences of Maastricht University within the NUTRIM School for Nutrition, Toxicology and Metabolism and the CAPHRI School for Public Health and Primary Care.

The research described in this thesis was funded by a grant from The Netherlands Organization for Health Research and Development (ZonMW; project number: 123000002).

Publication and distribution of this thesis was financially supported by Maastricht University, Open University of the Netherlands (Faculty of Psychology and Educational Sciences), Maastricht Instruments B.V. and HillHase Research and Development.

Cover design Brenda Berendsen

Layout Brenda Berendsen and Ridderprint B.V.

Printing Ridderprint B.V.

ISBN 978-94-6299-325-9

C) Brenda Berendsen, Maastricht 2016

All rights reserved. No parts of this publication may be reproduced or used in any manner whatsoever without prior written permission from the author. 


\title{
Measurement and promotion of physical activity
}

\section{Evaluation of activity monitors and a multidisciplinary lifestyle intervention in primary care}

\author{
PROEFSCHRIFT \\ Ter verkrijging van de graad van doctor \\ aan de Universiteit Maastricht, \\ op gezag van de rector magnificus \\ Prof. dr. L.L.G. Soete \\ volgens het besluit van het College van Decanen, \\ in het openbaar te verdedigen \\ op 24 juni 2016 te Maastricht \\ om I4.00 uur \\ door \\ Brenda Angela Juliette Berendsen \\ Geboren op I 3 januari 1987 te Heerlen
}




\section{Promotores}

Prof. dr. H.H.C.M. Savelberg

Prof. dr. N.C. Schaper

\section{Copromotor}

Dr. M.R.C. Hendriks

\section{Beoordelingscommissie}

Prof. dr. N.K. de Vries (voorzitter)

Prof. dr. em. M.A. van Baak

Prof. dr. G. Cardon (Universiteit Gent)

Prof. dr. A. Klink (Vrije Universiteit Amsterdam)

Prof. dr. T. van der Weijden 


\section{Contents}

Chapter I - General introduction

Chapter 2 - Which activity monitor to use? Validity, reproducibility and user friendliness of three activity monitors

Chapter 3 - A 20 minute window is optimal in a non-wear algorithm for tri-axial thigh-worn accelerometry in overweight people

Chapter 4 - Effectiveness and cost-effectiveness of BeweegKuur, a combined lifestyle intervention in the Netherlands: rationale, design and methods of a randomized controlled trial

Chapter 5 - The implementation and sustainability of a combined lifestyle intervention in primary care: mixed method process evaluation

Chapter 6 - Effectiveness of the BeweegKuur, a combined lifestyle intervention for 79 overweight people in primary care

Chapter 7 - General discussion 99

Valorisation

References

Summary

Samenvatting

Dankwoord

About the author 

CHAPTER I

General introduction 


\section{OVERWEIGHT AND PHYSICAL ACTIVITY}

Physical inactivity and excess body weight substantially contribute to the global burden of disease. In 2010 the global number of deaths attributable to these risk factors has been estimated to be 6.5 million (I). Excess body weight is closely related to cardio-metabolic disorders, such as insulin resistance, hypertension and high cholesterol, which are associated with an increased risk of diabetes mellitus and cardiovascular disease. In addition, patients with obesity are at risk for several non-vascular diseases such as cancer and osteoarthritis; obesity has therefore been acknowledged as one of the major risk factors for several non-communicable diseases (2). In $201342 \%$ of Dutch population were overweight $\left(\mathrm{BMI}>25 \mathrm{~kg} / \mathrm{m}^{2}\right)$, of which $10 \%$ were obese $\left(\mathrm{BMI}>30 \mathrm{~kg} / \mathrm{m}^{2}\right)$, and the prevalences of overweight and obesity are still increasing (3). Consequently, in the Netherlands, the health care costs attributable to overweight were approximately 1.6 billion euros in 2010 (4).

Causing weight gain, a lack of physical activity influences the risk for non-communicable diseases indirectly (2). Changes in body weight occur when energy intake and energy consumption are not balanced (2); a decrease in physical activity translates to low energy expenditure, and with an unchanged diet, this will lead to weight gain. On the other hand, research has revealed the critical role of physical activity in the development of metabolic disorders, regardless of weight $(5,6)$. This relationship between physical activity and health is mediated by enhanced cardiorespiratory fitness (6) and by activities of daily living (7). A large-scale observational study indicated that low cardiorespiratory fitness attributes to $16 \%$ of mortality (8). And regardless of fitness, recent research shows the crucial role of a physically active lifestyle besides exercise in cardio-metabolic health, e.g. light physical activity (7) and breaking up sitting time $(9,10)$. Moreover, sitting is associated with an adverse cardio-metabolic profile independent of physical activity level (I I-I3).

\section{COMBINED LIFESTYLE INTERVENTIONS}

Weight loss can be facilitated by pharmaceutical treatment, bariatric surgery and lifestyle coaching (14). But, most pharmaceutical options, as well as bariatric surgery, have a substantial risk for adverse events $(15,16)$ and do not necessarily improve lifestyle, disregarding the direct relationship between physical activity and cardio-metabolic risk profile. However, combined lifestyle interventions can treat overweight by targeting the underlying lifestyle behaviours that are responsible for excess weight, i.e. physical activity and diet, thereby also addressing the direct health benefits of a physically active lifestyle 
and a healthy diet. Besides physical activity, reduced energy intake can lead to weight loss and an improved cardio-metabolic risk profile (I7). In addition, quality of diet (e.g. fruit, vegetable and sugar-sweetened beverage consumption) has been related to weight gain $(18,19)$ and cardiovascular mortality $(20,21)$. Moreover, studies suggest that interventions aimed at improving healthy eating are cost-effective strategies to prevent cardiovascular disease and type 2 diabetes $(22,23)$ and to facilitate weight loss $(24)$.

As discussed above, it has been shown that physical activity is related to cardiovascular health. Reduced cardio-metabolic risk can be achieved by increasing moderate to vigorous physical activity (MVPA) $(25,26)$, but also by increasing physical activity at light intensity $(7,27)$. An increase of physical activity has also been shown to improve health related quality of life (28) and is expected to lead to cost savings in health care (29).

Evaluations of combined lifestyle interventions show different effects on lifestyle and health (30-36). The results are difficult to compare because each intervention includes a different type, amount and/or duration of guidance. In addition, the outcomes of these studies (e.g. behaviour, metabolic risk factors and quality of life) and time horizons are diverse (37). The Look AHEAD trial evaluated an intervention for overweight or obese participants with type 2 diabetes combining individual and group meetings with a lifestyle advisor, with weekly meetings during the first six months, bi-weekly meetings from six to twelve months and following up at least once every month for another three years (38). This high dose and long-term counselling led to sustained lifestyle changes and health benefits after four years, e.g. $45 \%$ increase in self-reported physical activity (34), 6\% weight loss and $0.4 \%$ reduction of $\mathrm{HbAlc}(33)$. Beneficial effects of the Look AHEAD intervention were also observed in quality of life, urinary incontinence, mobility and health care costs (3942). Yet, the intervention did not affect the incidence of cardiovascular events, the primary outcome of the Look AHEAD trial (43). In addition, feasibility of such a high dose programme in actual practice is questionable, because a higher dose of guidance requires more time and financial investments.

To find a balance between minimisation of costs and maximisation of effects, the optimal amount of guidance should be determined. Overall, combined lifestyle interventions (regardless of dose) seem effective to change lifestyle behaviour and thereby potentially improve health, when compared to a group receiving no or very little attention (e.g. one consultation or only information) (3I-35). In addition, lifestyle interventions have been suggested to be cost-effective in people with type 2 diabetes (44) and impaired glucose tolerance (45). But, some interventions of shorter duration and/or less guidance than the Look AHEAD trial yielded small or even no effects on lifestyle and weight $(30,36)$. So, the 
optimal dose of guidance to facilitate improvements in physical activity and diet is not yet clear.

\section{PRIMARY CARE SETTING}

In the Netherlands, health care is organised in primary and secondary care. Primary care providers can be contacted directly and are therefore easy to reach for people. The main primary care providers are the general practitioner (GP), the physiotherapist, the dentist, the psychologist and the dietician. Secondary care is specialist care provided in hospitals (46). GPs have a prominent role in the Dutch health care system, as they are the 'gatekeepers' to specialist care, and are free to access owing to reimbursement by the Dutch health care insurance. GPs often work in a practice in collaboration with a practice nurse, who is mainly responsible for prevention and management of chronic diseases (e.g. by providing three monthly consults with patients with type 2 diabetes). All Dutch citizens are subscribed with a GP practice in their own neighbourhood, implying a long-standing relationship with the practice and good accessibility of GPs (47). Therefore, GPs are able to diagnose and address overweight and obesity. In addition, health care providers in primary care should have a principal role in overweight treatment in order to prevent secondary health care use $(48,49)$. In particular the practice nurse has been hypothesised to play the key role in combined lifestyle interventions $(50)$.

Over the years, effectiveness of many combined lifestyle interventions for overweight has been studied. Strictly controlled clinical trials are crucial to accurately assess efficacy and working mechanisms, and several well-known examples exist (34, 5I). Nevertheless, besides efficacy trials in a controlled research setting, studies should be executed to assess effectiveness and cost-effectiveness in real-life settings (52). The successful implementation of innovations, such as a combined lifestyle intervention, relies on various factors, such as adaptability of the intervention and implementation strategy (53). Thus, the adaptation and evaluation of an intervention in the real-life setting is key to generate practice-based evidence. Such an approach is potentially superior to evidence-based practice for realistic expectations of effectiveness and for successful implementation of combined lifestyle interventions (52). However, only little research has studied the process, effectiveness and cost-effectiveness of combined lifestyle interventions that have been implemented in actual primary care. The studies that do exist have inconsistent results $(30,32,36,48,54,55)$. 


\section{THE BEWEEGKUUR PROGRAMMES}

In 2007, the Netherlands Institute for Sport and Physical Activity (NISB) was commissioned by the Dutch ministry of Health, Welfare and Sports to develop an intervention with exercise on prescription, the BeweegKuur $(56,57)$. The BeweegKuur is a combined lifestyle intervention set in Dutch primary care and is aimed at improving physical activity and diet in people with overweight or obesity. The intervention is based on the Self-Determination Theory that describes three requirements for a shift from external motivation to intrinsic motivation, i.e. competence, autonomy and relatedness, (58). Sustained behaviour change is more likely when people are intrinsically motivated (59). Lifestyle coaching by means of Motivational Interviewing addresses the basic needs of competence and autonomy. In addition, group sessions support relatedness to others (60). In the BeweegKuur, behaviour change is facilitated during one year by a GP, a lifestyle advisor (LSA), a physiotherapist and a dietician. The Beweegkuur consists of three different programmes and depending on BMI and co-morbidities participants can partake in one of these three programmes (tables I.I and I.2). The programmes differ in terms of amount of guidance by the physiotherapist, as people with a higher weight related health risk might perceive more barriers towards physical activity (32). Participants with a BMI of 25-35 $\mathrm{kg} / \mathrm{m}^{2}$ and no comorbidities are included in the so-called independent exercise programme, consisting of six meetings with the LSA, three individual and seven group meetings with the dietician and one consultation with the physiotherapist. Participants with a BMI of $25-35 \mathrm{~kg} / \mathrm{m}^{2}$ combined with risk factors for cardiovascular disease and type 2 diabetes (e.g. dyslipidaemia and impaired glucose tolerance) are included in the so-called start-up programme, that is similar to the independent programme, but with an additional five individual meetings with the physiotherapist to discuss barriers towards a physically active lifestyle. The participants with very high weight related health risk have a BMI of 25 $40 \mathrm{~kg} / \mathrm{m}^{2}$ combined with risk factors for cardiovascular disease or type 2 diabetes or are diagnosed with comorbidities. These individuals are eligible for the so-called supervised exercise programme, which is similar to the start-up programme, but with an additional 26 to 34 group meetings in the first three months of the intervention in which the physiotherapist provides supervised exercise to increase participants' physical activity level and overcome barriers to adopt other activities. Group cohesion resulting from these group sessions in the supervised exercise programme is expected to increase relatedness, a requirement for developing intrinsic motivation (58), and enhance adherence $(6 \mathrm{I}, 62)$. 
Table I.I. Eligibility criteria for the BeweegKuur programmes.

\begin{tabular}{|c|c|c|c|c|}
\hline & Independent & Start-up* & Supervised* & \\
\hline BMI & $25-35 \mathrm{~kg} / \mathrm{m}^{2}$ & $25-35 \mathrm{~kg} / \mathrm{m}^{2}$ & $25-35 \mathrm{~kg} / \mathrm{m}^{2}$ & $35-40 \mathrm{~kg} / \mathrm{m}^{2}$ \\
\hline $\begin{array}{l}\text { Presence of } \\
\text { comorbidities }\end{array}$ & No comorbidities & $\begin{array}{l}\text { Risk factors CVD } \\
\text { and } \mathrm{DM} 2\end{array}$ & $\begin{array}{l}\text { Diagnosed } \\
\text { CVD, DM2, } \\
\text { arthritis or } \\
\text { sleep apnoea }\end{array}$ & $\begin{array}{l}\text { No } \\
\text { comorbidities }\end{array}$ \\
\hline
\end{tabular}

$\mathrm{BMI}=$ body mass index; CVD = cardiovascular disease; DM2 = type 2 diabetes.

*Studies described in this thesis compared the start-up and supervised programme in participants eligible for the supervised programme (very high weight related health risk).

Table 1.2. Number of meetings with each health care professional in the BeweegKuur programmes.

\begin{tabular}{|c|c|c|c|}
\hline & Independent & Start-up* & Supervised* \\
\hline \multicolumn{4}{|l|}{ LSA } \\
\hline Individually & 6 & 6 & 6 \\
\hline \multicolumn{4}{|l|}{ Dietician } \\
\hline Individually & 3 & 3 & 3 \\
\hline Group-based & 7 & 7 & 7 \\
\hline \multicolumn{4}{|l|}{ Physiotherapist } \\
\hline Individually & 1 & 6 & $6-7$ \\
\hline Group-based & - & - & $26-34$ \\
\hline
\end{tabular}

LSA = lifestyle advisor.

*Studies described in this thesis compared the start-up and supervised programme in participants eligible for the supervised programme (very high weight related health risk).

Initially, the Beweegkuur intervention was aimed at people with type 2 diabetes or impaired glucose tolerance (pre-diabetes) (57). But, a literature and modelling study argued that the intervention would also be cost-effective in a population with overweight, potentially preventing type 2 diabetes (63). Hence, in this population, the independent and start-up exercise programme have been suggested to be effective strategies to enhance physical activity and improve health (63), and have been recommended to be included in Dutch basic health insurance (64). Whether reimbursement of the additional guidance of the supervised exercise programme (i.e. 26-34 group meetings) should also be considered by government and health insurers depends on the effects and costs compared to the startup exercise programme in the specified target group. Therefore, a multi-centred, clustered randomised controlled trial has been set up which is part of this thesis, 
comparing the supervised programme with the start-up programme, in participants eligible for the supervised programme.

\section{PHYSICAL ACTIVITY MEASUREMENT}

The assessment of physical activity is gaining attention in epidemiological research, in evaluation of lifestyle interventions and as feedback tool in interventions. As described above, recent studies have revealed a considerable role of physical activity and sedentary behaviour on health, independent of body weight (5-7). Unfortunately, these studies mostly rely on often-used self-report questionnaires, which are easy to use in large groups, increasing comparability between studies. However, they suffer from recall bias and social desirability, which might affect the validity of the results. Several technological devices have been developed in order to provide low-cost and objective alternatives to questionnaires being applicable in daily living, such as pedometers and accelerometers. Pedometers estimate the number of steps taken simply by counting the number of movements of a suspended lever arm that moves when the hip moves up and down with each step (65). Pedometers are cheap devices, but applicability in research is limited, as they do not discriminate between intensities of physical activity and daily step count is the only output (65). Accelerometry is a technique that measures accelerations (usually in three directions, i.e. tri-axial) and that can be applied in an activity monitor for the measurement of movement of the human body, i.e. estimating physical activity (66). By analysing the three-dimensional raw accelerations, physical activity can be estimated in detail taking activity intensity into account (66). In the past years, several types of activity monitors have been used, ranging in size, placement on the body, application method, data treatment and outcomes, and also providing discrimination between postures (e.g. sitting and standing). The posture discrimination in particular is a promising functionality, as research into sedentary time is gaining more and more attention due to its relationship with cardio-metabolic risk profile and mortality (independent of physical activity) (II-I3). Although it is tempting to estimate effectiveness of interventions by measuring physical activity and sedentary time with activity monitors, knowledge about their validity, reliability and user friendliness is often lacking, endangering accurate conclusions. Applying activity monitors with low validity leads to invalid estimates, and low reliability sabotages intervention studies with pre-post measurements. Moreover, low user friendliness might cause non-compliance of wearing activity monitors, thereby affecting validity and reliability of the output, even though the device might have been perfectly valid and reliable in a controlled laboratory setting (65). In addition, to interpret the complex output of an 
accelerometer several parameters for data treatment have to be defined. Examples of such parameters are epoch length (varying from one second to one minute), non-wear time definition, minimal number of days, activity cut off points and a sedentary cut off point $(67,68)$. Especially in an inactive, sedentary population the non-wear time algorithm can have substantial influence on outcomes, as the output of sedentary time and non-wear time is very similar (69). Therefore, effects of data treatment on the main outcomes should be well studied, before using activity monitors in research.

\section{GENERAL OUTLINE OF THIS THESIS}

This thesis aimed to evaluate

I) the quality of estimates of physical behaviour by accelerometers, and

2) the effectiveness of additional guidance in the BeweegKuur intervention executed in primary care in the Netherlands.

The studies regarding the measurement of physical activity are described in chapters 2 and 3. Chapter 2 presents the validity, reliability and user friendliness of three accelerometers which are used in scientific research. In chapter 3 we revealed the crucial role of a valid algorithm to extract non-wear time from measurements by an accelerometer.

Chapters 4 to 6 are devoted to the studies concerning the BeweegKuur lifestyle intervention. Firstly, chapter 4 describes in detail the design of the RCT to assess the additional effects of extra guidance aimed at physical activity. The process evaluation in chapter 5 critically investigated the execution and sustainability of the BeweegKuur in the primary care setting. Chapter 6 presents the effectiveness of the additional guidance by the physiotherapist in the BeweegKuur.

The thesis concludes with a discussion of the results in relation to existing literature and the strengths and limitations of the presented studies. 


\section{CHAPTER 2}

Which activity monitor to use? Validity, reproducibility and user friendliness of three activity monitors

Brenda AJ Berendsen

Marike RC Hendriks

Kenneth Meijer

Guy Plasqui

Nicolaas C Schaper

Hans HCM Savelberg

BMC Public Health 20I4, 14:749 


\section{Abstract}

Background Health is associated with amount of daily physical activity. Recently, the identification of sedentary time as an independent factor, has gained interest. A valid and easy to use activity monitor is needed to objectively investigate the relationship between physical activity, sedentary time and health. We compared validity and reproducibility of physical activity measurement and posture identification of three activity monitors, as well as user friendliness.

Methods Healthy volunteers wore three activity monitors simultaneously: ActivPAL3, ActiGraphGT3X and CAM. Data were acquired under both controlled $(n=5)$ and freeliving conditions $(n=9)$. The controlled laboratory measurement, that included standardised walking intensity and posture allocation, was performed twice. User friendliness was evaluated with a questionnaire. Posture classification was compared with direct observation (controlled measurement) and with diaries (free living). Accelerometer intensity accuracy was tested by correlations with walking speed. User friendliness was compared between activity monitors.

Results Reproducibility was at least substantial in all monitors. The difference between the two CAM measurements increased with walking intensity. Amount of correct posture classification by ActivPAL3 was $100.0 \%$ (kappa 0.98), 33.9\% by ActiGraphGT3X (kappa 0.29 ) and $100.0 \%$ by CAM (kappa 0.99). Correlations between accelerometer intensity and walking speed were 0.98 for ActivPAL3, 1.00 for ActiGraphGT3X and 0.98 for CAM. ICCs between activity monitors and diary were 0.98 in ActivPAL3, 0.59 and 0.96 in ActiGraphGT3X and 0.98 in CAM. ActivPAL3 and ActiGraphGT3X had higher user friendliness scores than the CAM.

Conclusion The ActivPAL3 is valid, reproducible and user friendly. The posture classification by the ActiGraphGT3X is not valid, but reflection of walking intensity and user friendliness are good. The CAM is valid; however, reproducibility at higher walking intensity and user friendliness might cause problems. Further validity studies in free living are recommended. 


\section{Introduction}

Growing evidence shows the negative influence of both physical inactivity and sedentary behaviour on health. It has been estimated that physical inactivity is currently related to $6 \%$ of mortality and is the main cause of $21-30 \%$ of several chronic diseases globally (70). In addition, an Australian study suggested that $7 \%$ of deaths were attributable to prolonged sitting (II). Recent studies suggest that an increase of physical activity could reduce metabolic risk independent of weight loss or aerobic fitness $(5,27)$. In line with this, an increasing amount of evidence reveals an independent association between sedentary behaviour and various health outcome measures (I I-I3). However, the optimal amount, frequency and intensity of physical activity and the maximum amount and optimal distribution of sedentary time are still a matter of debate.

Reliable and valid measurements of physical activity and sedentary behaviour are essential to draw sound conclusions about their influence on health. However, studies aimed at measuring sedentary behaviour have often used self-reported data that suffer from subjectivity (7I-73). Both reproducibility and validity of self-report physical activity and sedentary behaviour are variable $(7 \mathrm{I}, 74)$. Accelerometry has been proposed as a method to objectively quantify sedentary behaviour in addition to generally used measures of physical activity $(75,76)$. Generally, accelerometers present counts per minute as an intensity outcome based on the accelerations. Previously, the counts per minute output has been tested and used to estimate sedentary time and activity $(77,78)$. A problem of this approach is the inability to discriminate between sedentary time and standing time $(79,80)$. Recently, several tri-axial activity monitors have been developed that enable measurement of posture (e.g. sedentary behaviour and standing) by means of an inclinometer. The ActivPAL3 TM (AP; PAL Technologies Ltd, Glasgow, UK), ActiGraphGT3X (AG; ActiGraph LLC, Pensalcola, FL, USA) and CAM (Maastricht Instruments BV, Maastricht, NL) are activity monitors which measure physical activity intensity, register time spent in different postures (e.g. lying, sitting and standing) and thereby assess sedentary time. The AP and the AG have often been used in epidemiological studies, whereas the CAM is a new device developed to provide raw acceleration data. Reproducibility and validity of this inclinometer function has rarely been studied. The posture classification by the CAM was validated in patients with chronic obstructive pulmonary disease and chronic heart failure in daily routine at home (8I). The inclinometer function of the AG showed limited validity and a dependence on location of application (hip vs. back) $(77,82)$. Although several validation studies of the inclinometer function of the earlier manufactured uniaxial AP showed good posture classification (78, 
$80,83-85)$, we are not aware of a study aimed at the validity of the posture classification function of the tri-axial AP.

The validity and reliability of accelerometry measurements rely on wearing time (65). However, the required hours per day and total days of measurement are not always met by all participants, which will lead to exclusion of data. Sufficient wearing comfort is a crucial factor in compliance and can consequently affect data quality and validity $(66,68)$. Consequently, assessment of wearing comfort and attachment difficulty has been advised (8I).

The aims of this study were to assess I) reproducibility and validity of walking intensity and the posture classification of the AP, AG and CAM under laboratory conditions; 2) concurrent validity of the AP, AG and CAM with an activity diary in free living and 3) user friendliness of the three activity monitors.

\section{Methods}

Design

Data were acquired in both controlled and free-living measurements. In the laboratory measurement we compared data with observation, the gold standard; while the free-living measurements provided information in real daily life activities. In the laboratory measurement, the participants were instructed to follow a strict activity and posture protocol in a fixed setting. In the free-living measurement, participants were instructed to write down their activities in a diary every 15 minutes while wearing the devices in daily living. All participants completed a user friendliness questionnaire directly after the laboratory measurement or after returning the activity monitors when participating in the free-living measurement.

\section{Participants}

A convenience sample of 14 healthy adults with normal BMI participated in the study. Five of them participated in the laboratory measurement (4 male, I female, mean age 22.4 years \pm 2.2 ; mean BMI $22.3 \pm 1.8$ ); and nine participa ted in the free-living measurement (4 male, 5 female, mean age 27.2 years \pm 8.3 ; mean BMI $21.3 \pm 1.8$ ). Informed consent from participants was obtained. This study was approved by the ethics committee of Maastricht University Medical Centre. 


\section{Activity monitors}

In this study we assessed three tri-axial activity monitors: the ActivPAL3 (AP); the ActiGraphGT3X (AG); and the CAM (table 2.I). Both during the laboratory and in freeliving measurements, participants wore all three activity monitors simultaneously. Wearing instructions were always provided by the main researcher.

The AP was taped to the skin at the thigh, using double adhesive PALstickies ${ }^{T M}$ in the laboratory measurement. In the free-living measurement, the AP was waterproofed and attached with a Tegaderm ${ }^{\mathrm{TM}}$ dressing (3M Healthcare, St. Paul, MN, USA); and participants were instructed not to remove it for sleeping or showering. The AG was worn at the waist by means of an elastic belt and the participants were instructed to wear it at their back. As the AG is not waterproof, the device was to be removed when there was a risk of getting wet and during sleeping. To process the AG data, the ActiLife low frequency extension was used. The CAM was worn in an elastic belt around the thigh; also this device was to be removed during sleeping and when there was a risk of getting wet, because it is not waterproof.

The AP and CAM classify time as sitting/lying, standing and activity. The inclinometer function of the AG classifies time as sitting, lying and upright. For the analyses of the activity monitors individually, we assessed all classifications provided. In addition, we used sitting/lying time and upright time as generic measures in the laboratory measurement, to allow comparison between the three activity monitors. Sitting/lying time was defined as lying and sitting postures (regardless whether sitting time was misclassified as lying and vice versa by the $A G$ inclinometer); and upright time was defined as all time spent in an upright orientation (regardless whether active time was misclassified as standing and vice versa by the $A P$ and $C A M)$. Besides the inclinometer function, the $A G$ also discriminates between static posture (lying, sitting and standing) and activity based on a cut point of 100 counts on the vertical axis. For the AG only, the validity of this cut point was assessed in the free-living measurement. 
Table 2.I. Characteristics of the activity monitors and software

\begin{tabular}{|c|c|c|c|}
\hline & ActivPAL3 & ActiGraph GT3X & CAM \\
\hline Size & $53 \times 35 \times 7 \mathrm{~mm}$ & $38 \times 37 \times 18 \mathrm{~mm}$ & $63 \times 45 \times 18 \mathrm{~mm}$ \\
\hline Weight & $15 g$ & $27 g$ & $100 \mathrm{~g}$ \\
\hline Placement & Thigh & Waist & Thigh \\
\hline Application & Adhesives & Elastic belt & Elastic belt \\
\hline Range & $2 \mathrm{G}$ & $3 G$ & $4 \mathrm{G}$ \\
\hline Sample frequency & $20 \mathrm{~Hz}$ & $30 \mathrm{~Hz}$ & $25 \mathrm{~Hz}$ \\
\hline Waterproof & Yes & No & No \\
\hline Software* & $\begin{array}{l}\text { ActivPAL software } \\
\text { version } 6.0 .2\end{array}$ & $\begin{array}{l}\text { Actilife } \\
\text { version } 5.10 .0\end{array}$ & $\begin{array}{l}\text { Custom Matlab } \\
\text { program }\end{array}$ \\
\hline Classifications & $\begin{array}{l}\text { Sitting/Lying } \\
\text { Standing } \\
\text { Stepping }\end{array}$ & $\begin{array}{l}\text { Lying } \\
\text { Sitting } \\
\text { Upright }\end{array}$ & $\begin{array}{l}\text { Sitting/Lying } \\
\text { Standing } \\
\text { Active }\end{array}$ \\
\hline Intensity measure & $\begin{array}{l}\text { Metabolic Equivalent } \\
\text { (MET) }\end{array}$ & Counts & $\begin{array}{l}\text { Integrated Magnitude } \\
\text { Area (IMA) }\end{array}$ \\
\hline Epoch length* & Is & Is & Is \\
\hline $\begin{array}{l}\text { Non-wear } \\
\text { classification* }\end{array}$ & No & $\begin{array}{l}\text { Yes } \\
\text { (inclinometer code) }\end{array}$ & No \\
\hline
\end{tabular}

* Device offers more options; the option selected in this study is presented.

Laboratory measurement

In the laboratory measurement, we assessed the intensity measure and the inclinometer function to discriminate postures of the three activity monitors. To determine test-retest reproducibility, a protocol of 19.5 minutes was carried out twice by all participants, with a maximum of one day between measurements. The protocol consisted of periods of lying, sitting, and standing, walking over ground and walking and running on the treadmill (figure 2.I). Instructions were given orally. Four minutes were spent in a lying position, of which one minute on the side, one minute prone and two minutes supine. The protocol included two separate periods of sitting still on a chair. Thirty seconds were spent in a standing position (two periods of 15 seconds) and participants walked over ground two times. Finally, seven minutes were spent on the treadmill, walking with a speed of $0.3 \mathrm{~m} / \mathrm{s}$ up to $3.0 \mathrm{~m} / \mathrm{s}$. Speed was increased with $0.3 \mathrm{~m} / \mathrm{s}$ every minute up to $1.5 \mathrm{~m} / \mathrm{s}$, followed by $2.0 \mathrm{~m} / \mathrm{s}$ and $3.0 \mathrm{~m} / \mathrm{s}$. In case of deviations from the protocol, time and nature of the deviations were registered and corresponding time periods were excluded from the analyses. The 
measurement included different posture allocations, leading to transition periods in the data in between the allocations. The devices were synchronised with the protocol and each other by means of jumping at the start of the measurement (CAM) or their internal clocks (AG and AP). To prevent inclusion of transition phases, the first and last ten seconds of the data of each condition were excluded; if the condition duration was 30 seconds or less, the first and last five seconds were excluded. In analyses, a total sitting/lying time of 300 seconds, a total standing time of 10 seconds, and a total time with walking over ground of 20 seconds were used of each laboratory measurement (figure 2.I). For the AP, for each treadmill walking speed the average intensity was calculated with the middle 30 seconds to exclude transition phases, because intensity data per 15 seconds was used. For the AG and CAM the middle 40 seconds of each treadmill walking speed were used to calculate average intensity.

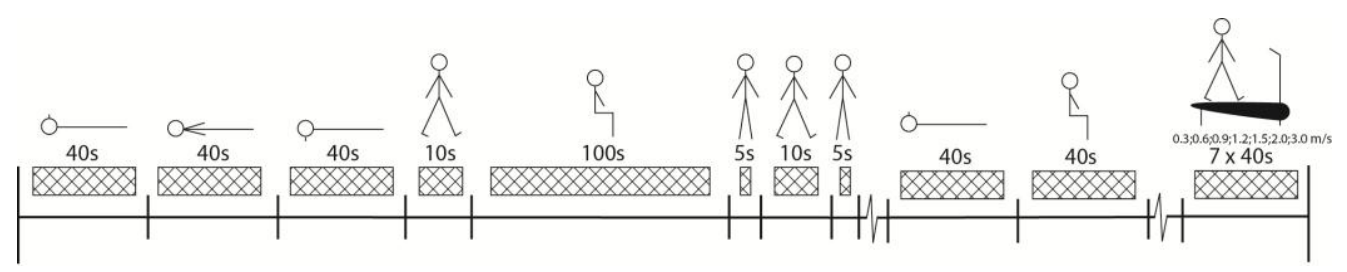

Figure 2.1. Composition of postures and activities of the protocol execution Legend: Postures (lying, sitting and standing) and activities (walking over ground and on treadmill) are depicted with corresponding included time blocks.

\section{Free-living measurement}

We evaluated four methods in the free living experiment (AP, AG inclinometer, AG counts and CAM). During the free-living measurement, participants wore the three activity monitors simultaneously for at least 3 days. All activity monitors were set to measure 24 hours per day. Participants filled out an activity diary every 15 minutes from waking up till going to bed, writing down the amount of minutes spent in four categories: sitting, walking, standing and other activities. These four categories were then classified as sitting/lying, standing and active. When activities occurred in only one category for longer than 15 minutes, participants were allowed to report them after the subsequent transition. Agreement with the diary was analysed per day. Minutes spent in each category were summed to a total day score. If the amount of minutes per hour registered in the diary exceeded or did not reach 60 minutes, minutes per category were normalised to match 60 minutes in total (referred to as corrected diary data). Both original and corrected diary data were used as comparator for the classification by the activity monitors in free living. 
User friendliness questionnaire

User friendliness was assessed in all participants with a self-administered questionnaire that was specifically developed for this study (table 2.2). The questionnaire consisted of eleven Likert-scale questions for each activity monitor and asked about their preferred activity monitor and were all completed directly after the measurement. The questions are summarised into five categories: self-positioning and removal (Cronbach's alpha 0.60), awareness of wearing (Cronbach's alpha 0.86), limitations in behaviour (Cronbach's alpha 0.75), advice and embarrassment. In all categories, a high score represents high user friendliness.

Table 2.2. The questions within each category of the user friendliness questionnaire

\begin{tabular}{|c|c|}
\hline Category & Questions \\
\hline $\begin{array}{l}\text { Self-positioning } \\
\text { and removal }\end{array}$ & $\begin{array}{l}\text { I. The activity monitor is easy to apply/position } \\
\text { 2. The activity monitor is easy to remove } \\
\text { 3. The activity monitor is difficult to apply (recoded) }\end{array}$ \\
\hline $\begin{array}{l}\text { Awareness of } \\
\text { wearing }\end{array}$ & $\begin{array}{l}\text { 4. The activity monitor fits easily underneath clothing } \\
\text { 5. I forgot I was wearing the activity monitor } \\
\text { 6. I noticed wearing the activity monitor while doing my daily activities } \\
\text { (recoded) }\end{array}$ \\
\hline $\begin{array}{l}\text { Limitations in } \\
\text { behaviour }\end{array}$ & $\begin{array}{l}\text { 7. The activity monitor limits me during my daily activities (recoded) } \\
\text { 8. The activity monitor limits me when l'm exercising (recoded) } \\
\text { 9. I've changed my activity pattern because of the activity monitor } \\
\text { (recoded) }\end{array}$ \\
\hline Advice & 10. I would recommend the activity monitor \\
\hline Embarrassment & $\begin{array}{l}\text { II. I would be ashamed if others would see I was wearing the activity } \\
\text { monitor (recoded) }\end{array}$ \\
\hline
\end{tabular}

\section{Analyses}

The reproducibility of posture classification during the laboratory measurement was analysed on a second-by-second basis with Cohen's kappa for nominal data, for each activity monitor individually. A kappa-value of $<0.4$ was defined as low agreement, $>0.4$ was moderate, $>0.6$ was substantial and $>0.8$ was almost perfect agreement (86). The reproducibility of the mean intensity of walking during the treadmill exercise was assessed with Intra Class Correlation (ICC) and Bland Altman plots.

Observation was used as gold standard in the laboratory measurement. Data from both laboratory measurements were pooled for validity analyses. Percentages of correctly 
classified seconds by each activity monitor were calculated and Cohen's kappa was used to test agreement with the protocol on a second-by-second basis. Friedman's ANOVA assessed whether the percentages of correctly classified sitting/lying and upright time differed between the three activity monitors. Correlations between walking speed and mean intensity per participant as provided by the standard software were calculated. Concurrent validity between posture classification by the activity monitors in the freeliving measurements and the diaries was assessed with ICC and Bland Altman plots. The CAM and AG were only worn during wake time; therefore, their analyses were performed on wake time diary data.

Differences in the category scores of user friendliness between activity monitors were tested with Friedman's ANOVA and Wilcoxon signed rank test (with an adjusted significance level of $p<0.0167)$. In addition, compliance in the free living measurement was registered.

Data were described as mean $\pm S D$; if data was not distributed normally, median and $25^{\text {th }}$ and $75^{\text {th }}$ percentile were calculated. Statistical analyses were performed with SPSS version 19 and with a two-tailed significance level of 0.05 (unless mentioned differently).

\section{Results}

\section{Laboratory measurements}

Test-retest reproducibility kappas of the posture classification function were 0.99 in AP, 0.75 in $A G$ and 0.95 in CAM (all $P<0.001$ ). Kolmogorov-Smirnov tests showed that almost all intensity data during treadmill walking of the three activity monitors were not distributed normally; therefore, Spearman's rho was used to assess test-retest reproducibility of the activity intensity during treadmill walking. The correlations between test and retest of the intensity measures were 0.97 in AP, 0.97 in AG and 0.96 in CAM (all $P<0.00 I$ ). Evaluation of Bland Altman plots revealed no systematic differences in the two measurements for both AP and AG; however, the differences between the two measurements of the CAM increased with larger intensity (figure 2.2a-c). 

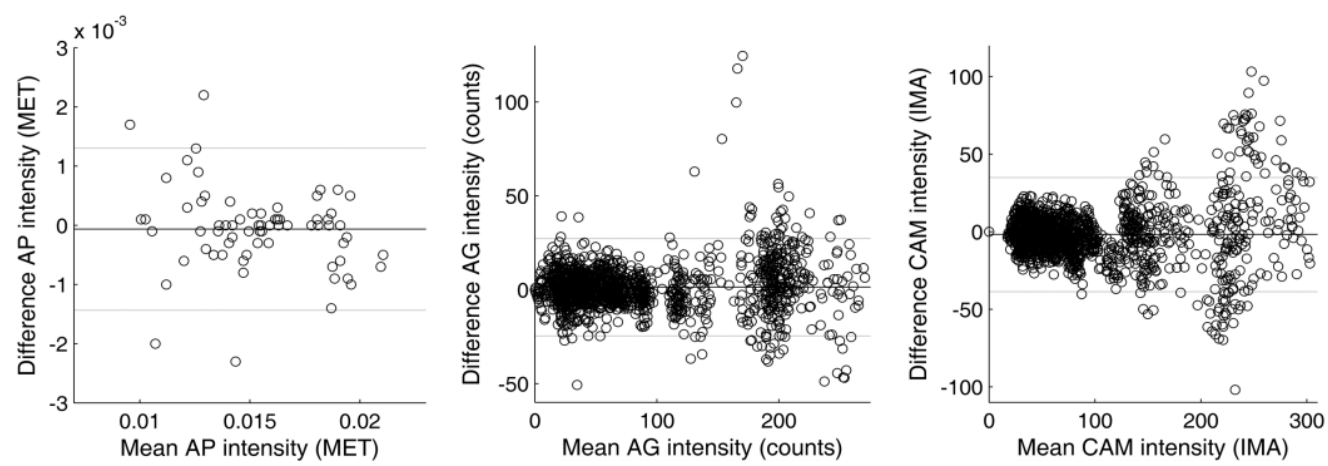

Figure 2.2a-c. Bland Altman plots for test and retest measurements of intensity, displayed for each activity monitor. Legend: Black lines display mean difference and grey lines display mean difference \pm 2 standard deviations. Mean difference and levels of agreement per activity monitor: AP $-0.1 \cdot 10^{-3}$ MET $\left(-1.4 \cdot 10^{-3}-1.3 \cdot 10^{-3}\right)$, AG I.4 counts $(-24.6-27.3)$ and CAM - I.7 counts $(-38.7-34.9)$.

Percentages of correctly classified time were not distributed normally; therefore, median values and $25^{\text {th }}$ and $75^{\text {th }}$ percentile were calculated. Sitting/lying, standing and walking time were classified correctly by the AP in $100.0 \%$ of the time with a kappa-value of 0.98 ( $<<$ 0.001 ) and the same categories were correctly classified by the CAM in $100.0 \%$ of the time with a kappa-value of $0.99(\mathrm{p}<0.00 \mathrm{I})$. Sitting, lying and upright time were classified correctly by the AG in $33.9 \%$ of the time with a kappa-value of 0.29 ( $p<0.001$ ) (specified for all categories and activity monitors in table 2.3).

Because of low correct posture identification, we looked at misclassification by AG in detail. In all participants a substantial amount of time spent lying was misclassified as non wear by the AG (table 2.4). In three participants the AG misclassified more than half of sitting time as upright. In one participant, sitting time was misclassified as both upright and non-wear. Overall $98.1 \%$ of classified non-wear occurred during lying, $1.7 \%$ occurred during sitting and $0.2 \%$ occurred during upright time.

Friedman ANOVAs showed that the ability to classify sitting/lying and upright time differed between the three activity monitors (sedentary: $p=0.010$; upright time: $p=0.007$ ), in which the AP and CAM performed similarly and the AG had a lower percentage of correct classification in both categories (table 2.3).

The validity analyses of the intensity measures resulted in ICCs of respectively $0.98(\mathrm{Cl}$ : $0.97-1.00)$, I.00 (Cl: $1.00-1.00)$ and $0.98(\mathrm{Cl}: 0.97-1.00)$ between the treadmill walking speed and mean intensity measures of the AP, AG and CAM (all $p<0.001$ ). 
Table 2.3. Correct classification in the laboratory measurement for each category specifically.

\begin{tabular}{lllll}
\hline & ActivPAL3 & ActiGraphGT3X & CAM & P-value \\
\hline Sitting/Lying & $100.0 \%$ & $35.7 \%$ & $100.0 \%$ & 0.010 \\
& $(100.0-100.0)$ & $(28.6-51.0 \%)$ & $(99.5-100.0 \%)$ & \\
Sitting & - & $33.9 \%$ & - & \\
Lying & - & $(0.0-83.2 \%)$ & & \\
& & $24.7 \%$ & - & 0.007 \\
Upright & $100.0 \%$ & $(22.5-32.2 \%)$ & & \\
& $(100.0-100.0 \%)$ & $(95.0-96.7 \%)$ & $(100.0-100.0 \%)$ & \\
Standing & $100.0 \%$ & - & $100.0 \%$ & \\
& $(100.0-100.0 \%)$ & & $(90.0-100.0 \%)$ & \\
Walking & $100.0 \%$ & - & $100.0 \%$ & $(100.0-100.0 \%)$ \\
& $(100.0-100.0 \%)$ & & $.99 *$ & \\
Cohen's kappa & $.98 *$ & $.29 *$ & & \\
\hline
\end{tabular}

Percentages are depicted as median $\left(25^{\text {th }}\right.$ percentile $-75^{\text {th }}$ percentile).

$* \mathrm{p}<.001$

Table 2.4. Classification of sitting and lying time by the ActiGraphGT3X in percentages for each participant ( $1-5)$.

\begin{tabular}{|c|c|c|c|c|c|c|}
\hline \multirow[b]{2}{*}{ Participant } & \multirow[b]{2}{*}{ Posture } & \multirow[b]{2}{*}{$\begin{array}{l}\text { Valid } \\
\text { seconds }\end{array}$} & \multicolumn{3}{|c|}{ Classified as: } & \multirow[b]{2}{*}{$\begin{array}{l}\text { Off } \\
\text { (non-wear) }\end{array}$} \\
\hline & & & Lying & Sitting & Upright & \\
\hline \multirow[t]{2}{*}{ I } & Lying & 315 & $22.5 \% \#$ & $1.3 \%$ & $1.9 \%$ & $74.3 \%$ \\
\hline & Sitting & 280 & $0.0 \%$ & $33.9 \% \#$ & $63.6 \%$ & $2.5 \%$ \\
\hline \multirow[t]{2}{*}{2} & Lying & 320 & $24.7 \% \#$ & $5.9 \%$ & $2.8 \%$ & $66.6 \%$ \\
\hline & Sitting & 280 & $0.0 \%$ & $0.0 \% \#$ & $100.0 \%$ & $0.0 \%$ \\
\hline \multirow[t]{2}{*}{3} & Lying & 320 & $59.1 \% \#$ & $0.6 \%$ & $3.1 \%$ & $37.2 \%$ \\
\hline & Sitting & 215 & $0.0 \%$ & $0.0 \% \#$ & $100.0 \%$ & $0.0 \%$ \\
\hline \multirow[t]{2}{*}{4} & Lying & 320 & $32.2 \% \#$ & $0.0 \%$ & $5.9 \%$ & $61.9 \%$ \\
\hline & Sitting & 265 & $0.0 \%$ & $98.9 \% \#$ & $1.1 \%$ & $0.0 \%$ \\
\hline \multirow[t]{2}{*}{5} & Lying & 310 & $16.5 \% \#$ & $5.5 \%$ & $3.9 \%$ & $74.2 \%$ \\
\hline & Sitting & 280 & $0.0 \%$ & $83.2 \% \#$ & $13.2 \%$ & $3.6 \%$ \\
\hline
\end{tabular}

\# Percentage correct classification 


\section{Free-living measurements}

During the free-living measurements one participant did not wear the AP and AG and one other participant did not wear the CAM. All activity monitors were worn for a mean of four days, ranging from two to six days per participant (eight participants). On average per person, three days of the AP-data were usable (seven participants). An average of four days of the AG could be used for the 100 counts cut off point (eight participants) and an average of two days could be used for the inclinometer analyses (seven participants). An average of three days of the CAM measurements could be used in analyses (eight participants). Reasons for missing data were: the AP did not register data, the AG inclinometer did not register data, the CAM did not register data, the CAM stopped measuring before midnight and diary data was incomplete.

To assess validity in free living, posture classification was compared with diary data. There appeared to be no difference in ICC-values between the comparisons with original diary data and with corrected diary data (to correct diaries not reaching 24 hours per day). ICC of the AP with the original and corrected diary outcomes was 0.98 (Cl: $0.94-0.99)$. ICC of the CAM was 0.98 ( $\mathrm{Cl}: 0.95$ - 0.99). Evaluation of Bland Altman plots revealed that according to AP and CAM, the total duration of activity was systematically lower and total duration of standing was systematically higher than in diaries (figures 2.3 and 2.4).

The ICC of the inclinometer function of the AG was $0.59(\mathrm{Cl}: 0.22-0.8 \mathrm{I})$, upright time was systematically higher and sitting time was systematically lower than in the diaries (figure 2.5). The distinction between static time and activity by the AG cut point of 100 counts had an ICC of $0.96(\mathrm{Cl}: 0.88$ - 0.98). The Bland Altman plots showed good agreement with diary, with exception of one participant in which static time was lower and active time was higher according to AG (figure 2.6).
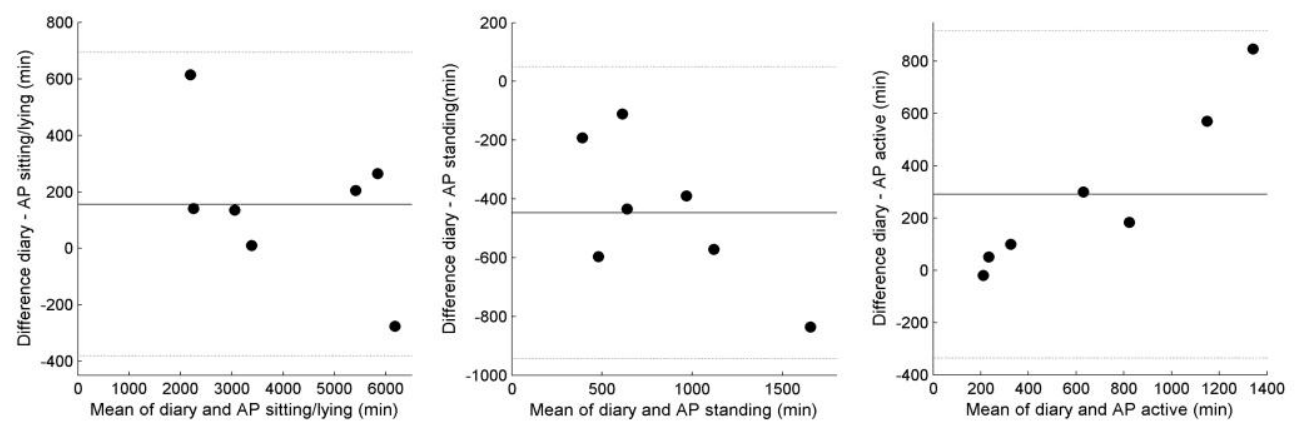

Figure 2.3a-c. Bland Altman plots of time registered in diaries and time classified by AP. Legend: Black lines display mean difference and grey lines display mean difference \pm 2 standard deviations. Mean difference and levels of agreement in minutes per category: Sitting/lying I56.6 (-38I.8 - 695.0), standing -447.I (-944.5 - 50.2), active 290.7 (-336.4- 917.8). 

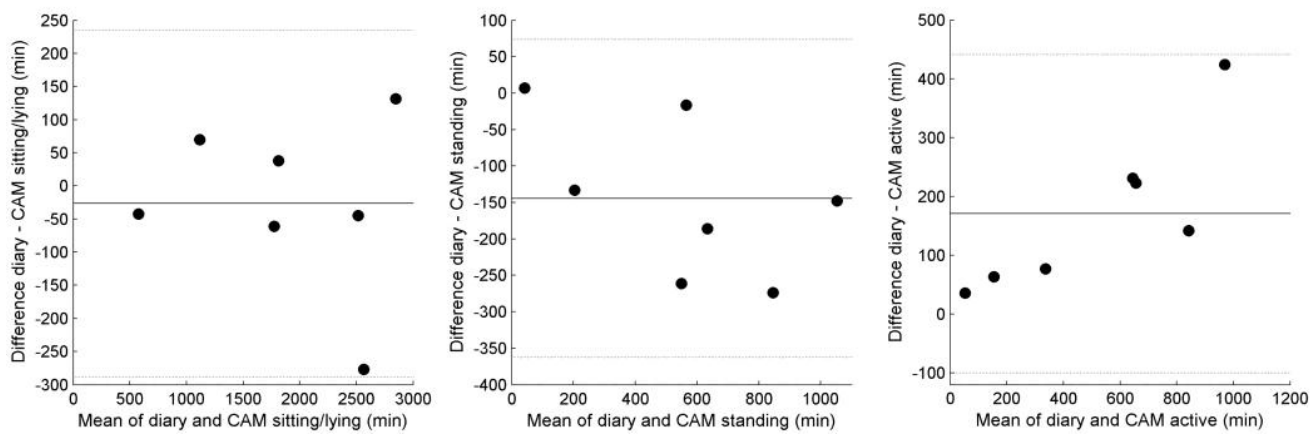

Figure 2.4a-c. Bland Altman plots of time registered in diaries and time classified by CAM.

Legend: Black lines display mean difference and grey lines display mean difference \pm 2 standard deviations. Mean difference and levels of agreement in minutes per category: Sitting/lying $-26.5(-288.3-235.4)$, standing - 144.5 ($362.8-73.8)$ and active $171.0(-99.8-441.7)$.
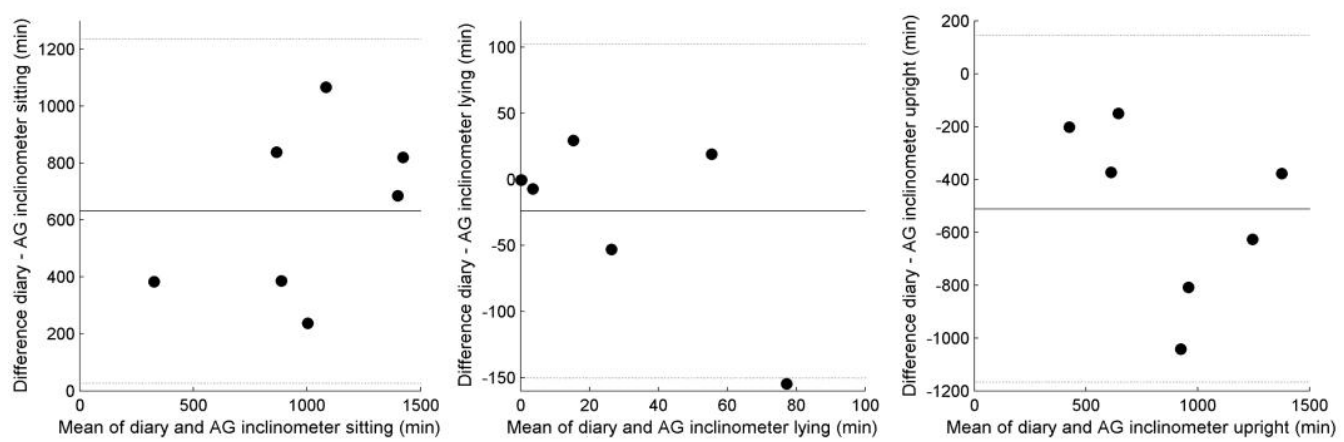

Figure 2.5a-c. Bland Altman plots of time registered in diaries and classified by AG inclinometer. Legend: Black lines display mean difference and grey lines display mean difference \pm 2 standard deviations. Mean difference and levels of agreement in minutes per category: Sitting 631.0 (26.2 - 1235.8), lying -23.9 (-I $50.3-$ 102.5) and upright -5। I.8 (-1 I67.2 - |43.6).
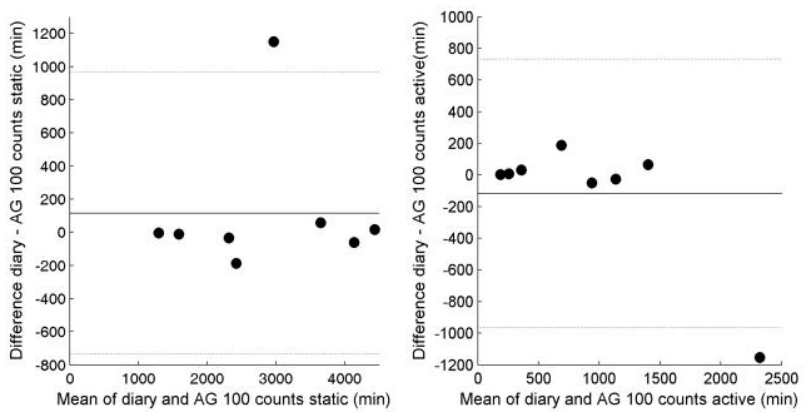

Figure 2.6a-b. Bland Altman plots of time registered in diaries and classified by AG counts.

Legend: Black lines display mean difference and grey lines display mean difference \pm 2 standard deviations. Mean difference and levels of agreement in minutes per category: Static I I 5.7 (-732.I - 963.6) and active - I I6.7 (-966.I $-732.7)$. 


\section{User friendliness}

The activity monitors had significantly different scores on all question categories according to Friedman's ANOVAs, except on the question regarding embarrassment to wear the devices (figure 2.7). A Wilcoxon signed-rank test revealed that the AG had higher scores, i.e. was easier to use, with respect to self-positioning and removal than the CAM and AP $(P=0.011$ and $P=0.003)$. On the awareness of wearing scale, the CAM scored significantly worse than the $A G$ and $A P$, implying that participants were more conscious about wearing the CAM than wearing the other devices $(p=0.01 \mathrm{I}$ and $\mathrm{p}=0.00 \mathrm{I})$. Participants experienced significantly more limitations in behaviour with the CAM compared to the AP; the CAM also had significantly lower scores than AP with regards to $\operatorname{advice}(p=0.008$ and $p=0.007)$.

One participant of the laboratory measurement preferred the AP and four preferred the AG. Seven participants in the free-living measurements preferred the AP and two preferred the AG. None of the participants indicated CAM as preferred activity monitor to wear.

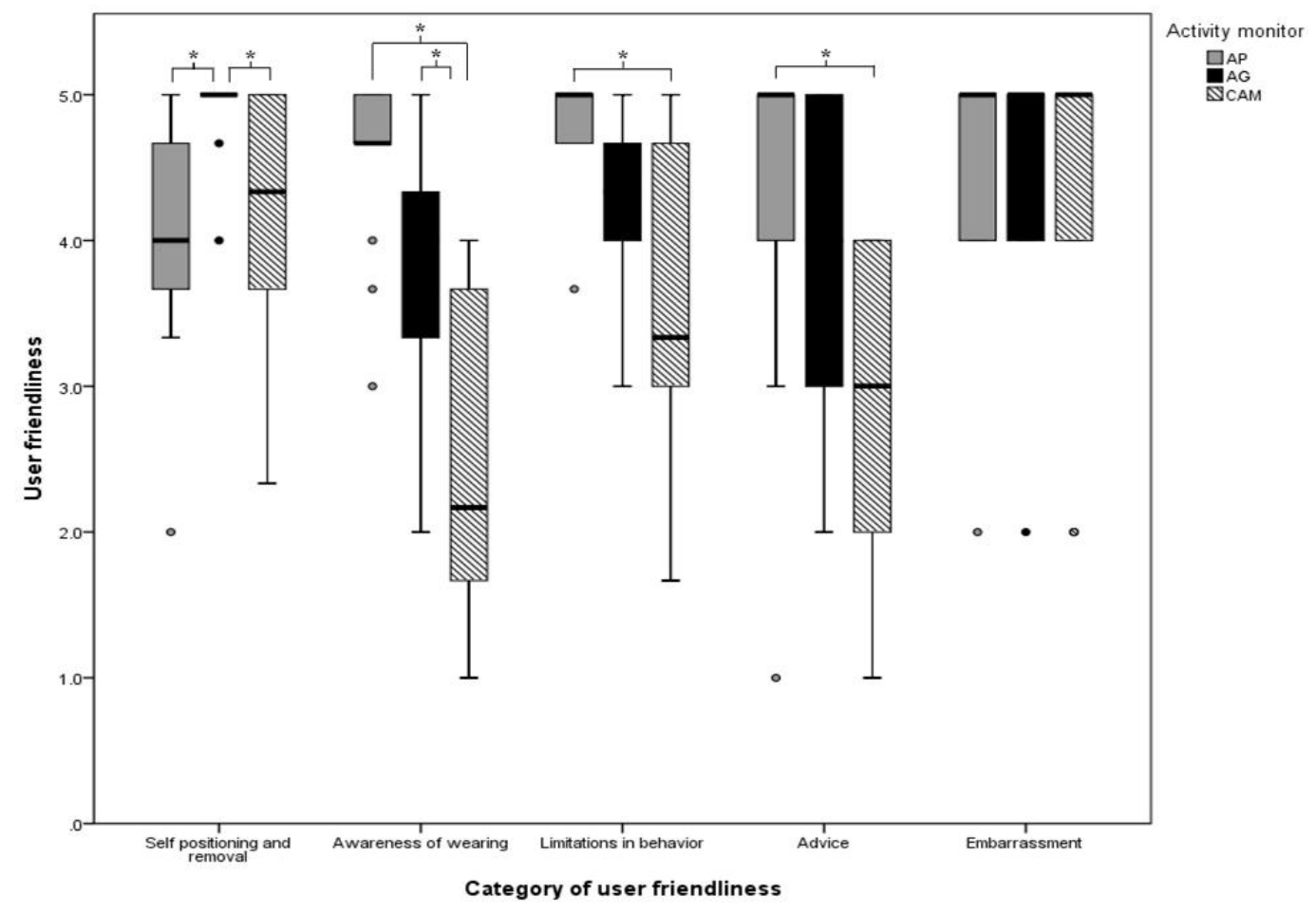

Figure 2.7. User friendliness scores of the activity monitors for each category. High scores represent high user friendliness. *Significantly different $(p<0.0167)$ 
During the laboratory measurement one participant found it uncomfortable to remove the AP after a short period of measuring and two participants commented that the elastic belt of the CAM was uncomfortable. Following user friendliness issues occurred during the free-living measurements: reported skin irritation due to adhesive material of the AP $(n=$ 3), AG was uncomfortable during sitting, lying or carrying a bag $(n=5)$, skin irritation due to the elastic belt of the CAM $(n=2)$, aching muscles due to the elastic belt of the CAM $(n=I)$, CAM was uncomfortable due to sweating while playing sports, not fitting under clothes and did not stay in place $(n=3)$.

\section{Discussion}

Choosing a suitable activity monitor for scientific studies depends on various aspects. This study aimed to address validity, reproducibility and user friendliness of three activity monitors available for measurement of physical activity and posture classification. Findings of our study indicate a trade-off between these three aspects in the AG and CAM. The AG shows moderate to high reproducibility but low validity for posture allocation and high user friendliness. The CAM shows moderate to high reproducibility, high validity, but low user friendliness. The AP scored well on all three aspects considered: high reproducibility, high validity and high user friendliness (despite reported skin irritation in four participants).

Both $A P$ and CAM showed very good estimations of sitting/lying, standing and walking time. The postures were almost always classified correctly, indicating high validity. Other studies have shown this as well for CAM $(8 I)$ and the uni-axial version of the AP $(78,80$, $84,85,87)$. The high reproducibility of the AP was in accordance with findings of a study aimed at the step counts of the uni-axial AP (88). In the current study, reproducibility of the activity intensity estimated by the CAM at higher walking speed might be insufficient. This raises the question whether the CAM is able to adequately estimate activity intensity at higher intensities, a prerequisite for the discrimination of moderate and vigorous physical activity in pre-post measurements. Bearing in mind that the reproducibility analyses included data of only five participants, the fixation of the CAM by means of the elastic belt might not be secure enough and may have caused the low reproducibility at higher activity intensity.

The ICCs confidence intervals of the AP, AG counts and CAM were acceptable. However, the confidence interval of the ICC of the AG inclinometer function was wide, limiting generalisability to the population level. In addition, plots showed that differences with diary registration were large, despite the moderate to high ICC-values of classification by the activity monitors in daily living. The design of the free living part of the study refrained 
us from concluding whether the discrepancies were caused by misclassification of the devices or by inaccuracy of the diary as comparator. Participants were asked to report their activities every 15 minutes, as this was believed to be both feasible and accurate. Participants made an effort to report their daily activities in detail (i.e. in minutes precise). Nevertheless, reporting accuracy remains an issue which was not controlled for.

The AG inclinometer did not perform well in terms of reproducibility and validity of posture classification in both the lab and the free-living measurement. The second-bysecond analysis of the laboratory measurement showed that much lying time is wrongly classified as non-wear by the inclinometer and sitting and upright time are often mingled. In addition, the amount and type of misclassification seems to be different between participants, for instance, in one participant $83.2 \%$ of sitting time was classified correctly, while sitting time in other participants was never classified correctly. The participants were instructed to wear the $A G$ at the back during the measurements in this study because acceleration data reflects physical activity best when the device is worn at the lower back (89). Although the AG manual states that the inclinometer function performs best when the AG is worn at the hip area, our findings are in line with the results of McMahon et al. (2010) who evaluated the validity of the inclinometer function when attached at back, waist and upper leg. The results of McMahon et al. indicated that compared to the waist, attachment to the back led to more correctly classified standing time and less correctly classified sitting and lying time. Moreover, neither attachment location led to sufficiently correct sitting and lying identification (82). Another study in which the AG was worn at the hip found correct posture classifications of $60.6 \%$ (standing) to $66.7 \%$ (lying). In that study, lying time, watching TV and sitting behind computer were also often classified as non-wear (respectively 14.3\%, 6.5\% and 9.3\%). Also, watching TV and sitting behind computer were often classified as standing time (30.1\% and $23.6 \%$ ) (77). Most remarkable is the amount of wrongly identified non-wear regardless of attachment location, especially in lying time. In our study, we adopted the non-wear classification provided by the inclinometer function. Usually, non-wear is identified with an algorithm based on a certain amount of inactivity $(69,90,91)$. These algorithms have been proven to be sufficiently valid to recognise non-wear in AG measurements (69). Therefore, it might be advisable to reconsider the added value of the non-wear classification based on inclinometer data. In contrast to the inclinometer function, the discrimination of static and active time based on the cut point of 100 counts on the vertical axis was good. This is in agreement with previous studies $(77,78)$, which shows that when amount of activity is point of interest, regardless of sedentary time, the AG provides valid data. 
Our user friendliness questionnaire addressed five aspects of which three have been proposed earlier. Application of activity monitors in free living requires a device that is easy to use, comfortable and unobtrusive $(66,68)$. The CAM scores lowest in most subscales. Possibly, low scores decrease compliance and affect reflection of (in)activity patterns, due to obtrusiveness. The obtrusiveness of the CAM might be higher than the other two devices because of the relatively large size of the CAM and the large elastic belt that was used to wear it. However, compliance of participants to wear the activity monitors in this study was equal. This implies that the application method, removable (CAM and $A G$ ) or taped to the skin (AP), does not relate to compliance of wearing. Certain characteristics or subscales of user friendliness might be less or more important dependent on the goal and design of the study. In short measurements, the AG is preferred, whilst the AP is preferred in measurements of several days, even though skin irritation was reported by some participants. Further work is needed to relate the user friendliness to wearing compliance and behavioural adaptations.

The relative small sample size is a limitation of the current study. In addition, the sample consisted of only normal-weight, healthy adults. Therefore, results cannot be generalised to clinical or overweight adults and the user friendliness questionnaire should be assessed for validity and reproducibility in a larger, more variable population. Another limitation is the aforementioned lack of direct observation during free-living measurements. MachadoRodrigues et al. showed that a more detailed diary yielded valid results against an accelerometer (92). However, diaries always suffer from approximation and although participants were instructed to fill in their diary continuously, we could not control for recall bias in case of non-compliance. Therefore, it is not possible to draw solid conclusions about construct validity from these findings. Nevertheless, by including both controlled laboratory measurements and free-living measurements, our results give an indication of the reproducibility, validity and user friendliness of the three activity monitors. 


\section{Conclusion}

Results of activity monitoring depend on the device used, and choice of device should depend on the research aims and design. The majority of the studies which led to the current consensus on the negative influence of sedentary time on health, independent of physical activity, are based on subjective measures. As an objective measure, accelerometry can reinforce earlier results. The current study shows that the AP and CAM are able to classify posture and that the inclinometer function of the AG provides no valid posture classification. However, the AG can well be used if level of physical activity is of interest. 


\title{
CHAPTER 3
}

\begin{abstract}
A 20 minute window is optimal in a non-wear algorithm for tri-axial thigh-worn accelerometry in overweight people
\end{abstract}

Brenda AJ Berendsen

Marike RC Hendriks

Paul Willems

Kenneth Meijer

Nicolaas C Schaper

Hans HCM Savelberg

Physiological Measurement 2014, 35:2205-2212 


\section{Abstract}

Introduction A valid non-wear algorithm for activity monitors is crucial to avoid the misclassification of sedentary time as non-wear time, and vice versa. Characteristics of the algorithm, such as time windows, should be well defined and tested. Furthermore, using tri-axial data might influence the algorithm's performance. This study assessed the optimal time window length in a non-wear algorithm for overweight adults, applied to tri-axial data from sixteen participants.

Methods Ten time windows, from 10 up to $120 \mathrm{~min}$, were tested with a diary as a criterion measure. We assessed the bias in non-wear time, sensitivity and specificity. The optimal time window length was based on ten participants; the validation of this time window was carried out with six other participants.

Results The time window of 20 min showed the highest and 120 min showed the lowest mean amount of correctly classified non-wear time, at $94 \%$ and $70 \%$ respectively. Sensitivity and specificity were considered optimal in the 20 min time window. Validation of this time window demonstrated a sensitivity and specificity of $86 \%$ and $83 \%$ respectively.

Conclusion A 20 min time window showed the best non-wear estimations. The current study utilised tri-axial raw data and I s epoch data which might have facilitated the application of a short time window and thereby decreased the risk of misclassifying non wear. 


\section{Introduction}

Although accelerometry offers an objective measure of physical behaviour (e.g. time spent sitting or being physically active), accuracy relies on the compliance of wearing the activity monitor. For various reasons, such as water-related activities, and sleeping, participants rarely wear the activity monitor continuously. To prevent the influence of non-wear on the physical behaviour output, non-wear time should be removed from the data before analysing the activity monitor measurements. The most commonly used algorithm classifies time as non-wear only if zero counts on the vertical axis are registered for at least 60 consecutive minutes, with allowances for $1-2$ min with counts between 0 and 100 $(90,93)$. This algorithm has also been used for NHANES data measured with a uniaxial ActiGraph worn at the waist (90). However, one non-wear algorithm cannot be applied to other activity monitors with other placements or populations without validation.

Manipulation of the time window results in different estimations of physical activity and inactivity, and therefore might affect conclusions drawn from measurements (69, 93-97). The lengths of time windows differ from 10 to $90 \mathrm{~min}$ for the algorithms utilised $(69,94)$. In addition, studies apply a minimum wear time of $10 \mathrm{~h} \mathrm{d-I}$ to consider a measurement valid (68). The utilisation of an invalid algorithm can falsely decrease or increase the number of valid days due to the misclassification of non-wear. It is difficult to avoid misclassification of non-wear, especially in sedentary populations such as the elderly, because both sedentary time and non-wear time show similar outputs. In addition, Winkler et al. (20I2) showed that body mass index (BMI) influences the performance of the generally accepted non-wear algorithm. Non-wear misclassification was significantly higher in overweight and obese persons compared to normal and underweight persons (9l), indicating that algorithms validated in normal weight persons might not be valid in overweight and obese persons. Thus, the length of the time window should be tested in the population of interest. If the study involves a population that is more likely to be sedentary, such as overweight or elderly persons, the time window should probably be longer to avoid the misclassification of sedentary time as non-wear time. Choi et al. (2012) found less misclassification in the elderly when using a time window of 90 min, compared to a time window of $60 \mathrm{~min}$. In addition, Hutto et al (2013) found longer time windows resulted in less misclassification of non-wear in older adults. However, a problem related to a larger time window is the increased risk of misclassification of short non-wear intervals (such as during showering) as wear time $(69,98)$. Unfortunately, conclusive evidence about the optimal time window length in overweight adults is lacking.

Until now, most non-wear algorithms have been based on activity counts on one axis (9I, 94-96, 99, 100). Since many activity monitors measure acceleration on three axes, applying 
a non-wear algorithm on tri-axial data might improve non-wear estimates. In a wrist-worn activity monitor, an algorithm based on three axes performed better in estimating nonwear when compared to the algorithm based on vertical counts only (69). In addition, as a wearing location the wrist was more sensitive in detecting wear and non-wear than the waist. The utilisation of three axes in the non-wear algorithm of the waist-worn activity monitor was not assessed. Therefore it is not known whether a non-wear algorithm on tri-axial data worn on locations other than the wrist might improve non-wear estimates.

Because the optimal time window is still unclear, and because including tri-axial data might improve the classification of non-wear, we aimed to find and validate the optimal time window in a non-wear algorithm in overweight and obese adults using the tri-axial CAM, worn at the thigh.

\section{Methods}

Sixteen participants in the BeweegKuur study $(\mathrm{IOI})$ were randomly selected. Participants were included by general practitioners, were overweight or obese, and wore the CAM (Maastricht Instruments BV, Maastricht, NL) (8I) for 5 consecutive days before participating in a combined lifestyle intervention. The CAM is a tri-axial accelerometer with a sample frequency of $25 \mathrm{~Hz}$ and a range of $4 \mathrm{G}$. The device was worn in an elastic belt around the thigh and had to be removed for sleeping and when there was a risk of getting wet. Participants were encouraged to wear the monitor continuously and to write down non-wear intervals in a diary, with reasons for non-wear and specific starting and ending times. The times in the diary were used to retrieve the exact start and end times of wear and non-wear intervals in the graphical representation of the accelerations. This method has previously been used by Choi et al. (20I2) to assess the accuracy of non-wear algorithms.

First, the total non-wear time was calculated based on the exact starting and ending times of the non-wear intervals, and used as a criterion measure. Second, the total measurement was divided into consecutive wear and non-wear intervals. These intervals were used to assess the sensitivity and specificity of the algorithms. The most optimal time window was determined based on the data from ten participants (mean age $54 \pm 15$ years; mean BMI $34.0 \pm 4.6 \mathrm{~kg} / \mathrm{m}^{2}$ ), and then validated with data from six other participants in the BeweegKuur study (mean age $63 \pm 8$ years; mean BMl $36.0 \pm 4.9 \mathrm{~kg} / \mathrm{m}^{2}$ ).

Data filtering and algorithm parameters

If counts remained below the CAM's noise level for 10 to $120 \mathrm{~min}$, time was classified as non-wear time, with an allowance of $60 \mathrm{~s}$ of counts between the noise level and the 
previously validated sedentary cut off point $(81,102)$. The duration of the time window was manipulated from 10 to $120 \mathrm{~min}$, in steps of $10 \mathrm{~min}$. After the classification of nonwear, the algorithm assessed the change in accelerations in the three axes between the start and end of each non-wear period. If this change reached a threshold, we assumed the accelerometer had slightly moved and the time was considered to be wear time.

\section{Analyses}

For both the optimisation and validation of the non-wear algorithm, we compared the criterion measure with non-wear intervals estimated by the algorithm. For each time window we assessed the bias in total duration of non-wear, defined as the total duration of the criterion measure minus the total duration of non-wear estimated by the algorithm. In addition, we analysed whether each wear and non-wear interval was correctly classified by the algorithm (with an allowed deviation of $5 \mathrm{~min}$ in the start and end time). Based on the classification per interval, sensitivity (true non-wear/all non-wear) and specificity (true wear/all wear) were calculated. A true positive was defined as a correctly classified nonwear interval; a true negative was defined as a correctly classified wear in terval. The most optimal algorithm would result in the least bias between our criterion measure and the algorithm, and have an acceptable sensitivity and specificity. Analyses were performed using a custom MATLAB program.

\section{Results}

Diaries showed that the mean non-wear time due to sleeping was $34.6 \pm 9.6 \%$ of the total time measured. Non-wear due to other reasons, such as showering and skin irritation, was $3.0 \pm 3.5 \%$ of the time. Besides for sleeping, the least number of removals during the entire measurement per participant was zero; the maximal number of removals was five; and the median was two removals. Because the CAM had to be removed for sleeping, non-wear intervals longer than 2 hours occurred most frequently. Of the non-wear intervals shorter than 2 hours, $65 \%$ were under I hour (figure 3.I). 


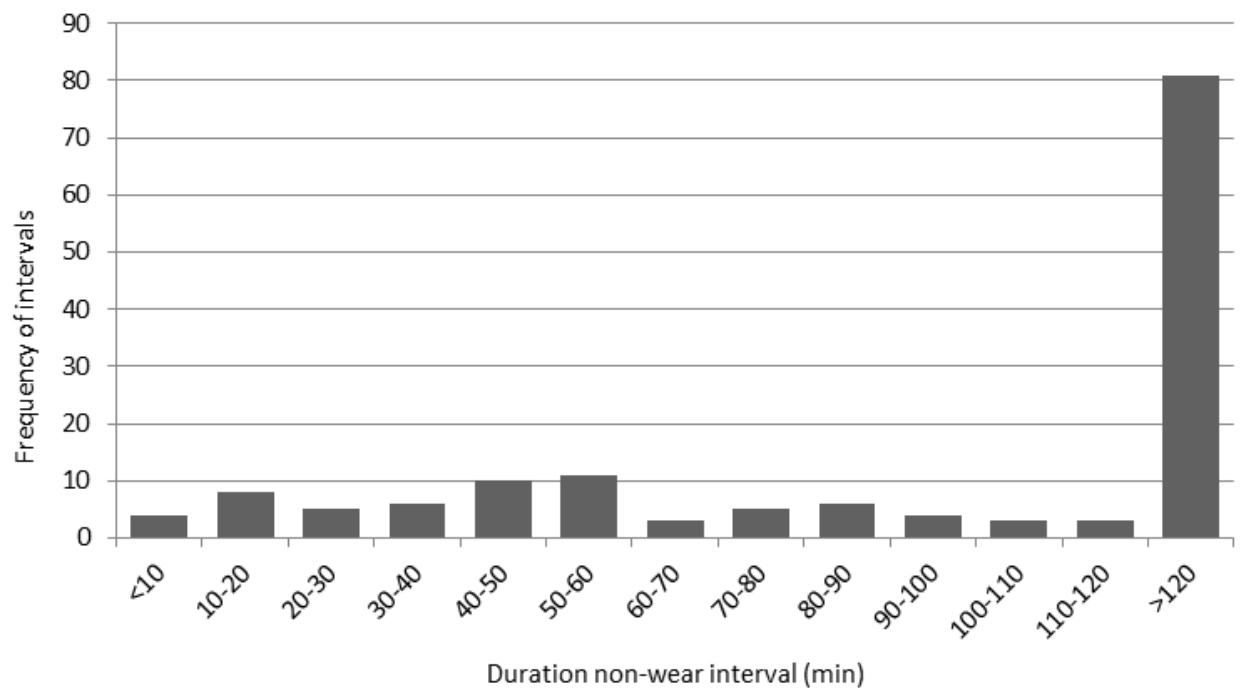

Figure 3.I. Frequency of non-wear intervals

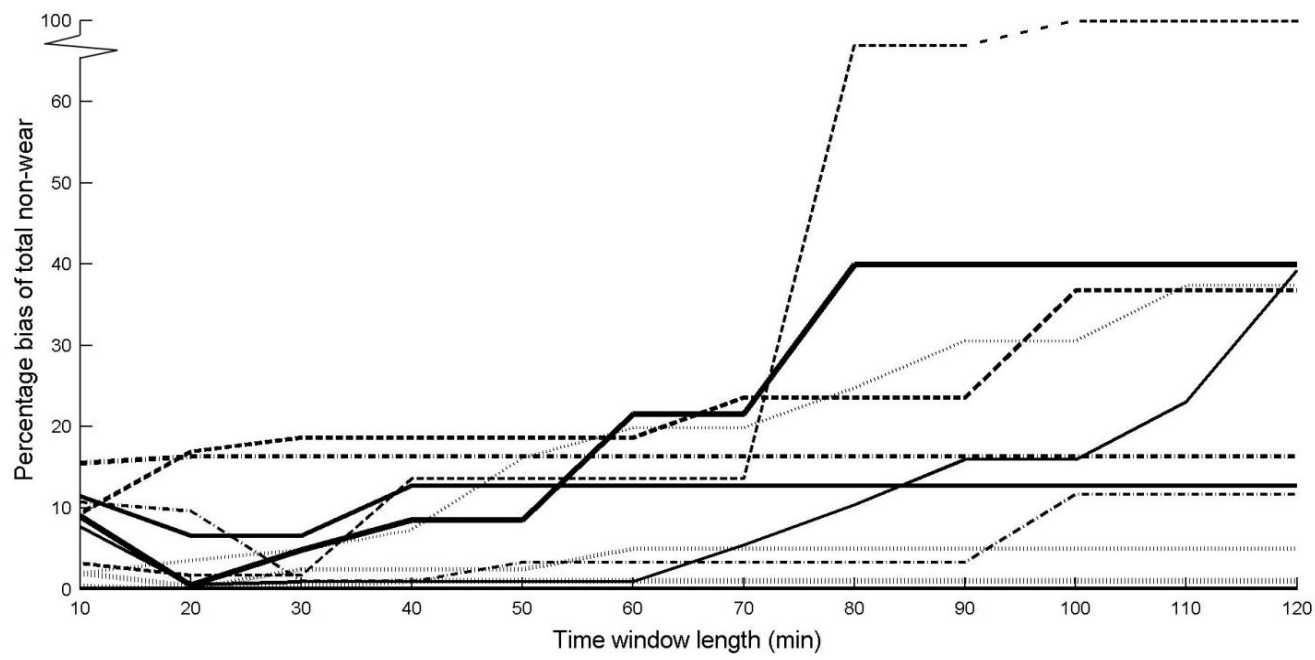

Figure 3.2. Percentage difference between algorithm and the criterion measure as a function of time window length, per participant (different lines). 
In most participants $(n=8)$, the time window of 10 or 20 min was optimal. In those participants, longer time windows led to decreases in the correctly classified non-wear time. In one participant all time windows resulted in the same amount of bias, and in one participant the $30 \mathrm{~min}$ time window was optimal for the estimation of total non-wear time. The mean percentage of non-wear that was correctly classified by the algorithm increased from $70.0 \%$ (range 0.0 - 99.0\%) with a time window of $120 \mathrm{~min}$, up to $94.4 \%$ (range 83.1 - 100.0\%) for a time window of $20 \mathrm{~min}$. The $10 \mathrm{~min}$ time window lead to 91.4\% (range 82.5 - 99.5\%) correctly classified non-wear time (figure 3.2).

With increasing time window lengths, the specificity increased from 59.3 to $95.0 \%$, and the sensitivity decreased from 89.0 to $47.3 \%$. The values of $(0 ; 0)$ and $(1 ; 1)$ were added in the ROC-curve for illustration, to represent $100 \%$ sensitivity and $0 \%$ specificity and vice versa (figure 3.3). The area under the curve was 0.83 . The percentage of correctly classified non-wear time and the combination of sensitivity and specificity were deemed best for the time window of $20 \mathrm{~min}$.

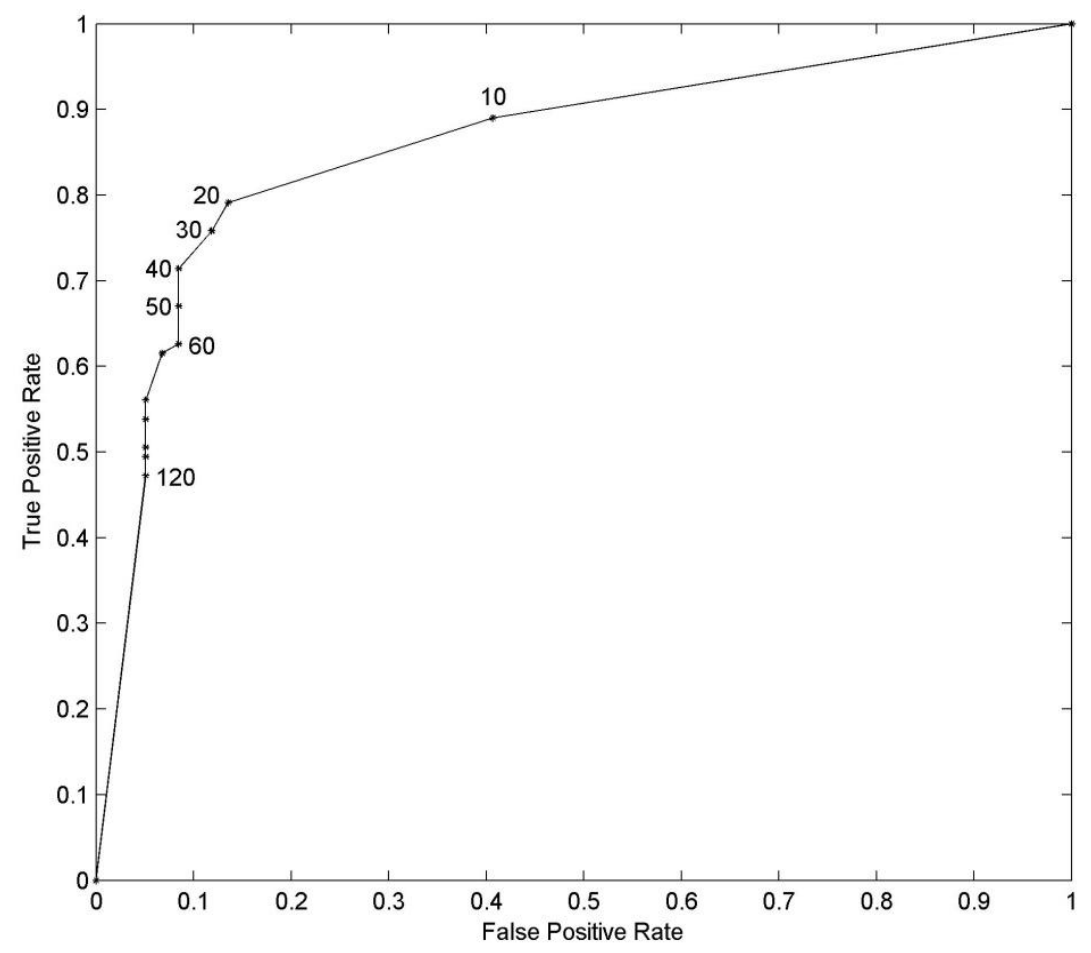

Figure 3.3. ROC-curve of the algorithm. The numbers next to the markers represent specific time windows 
The validity of the 20 min time window was assessed in six other overweight participants. The amount of non-wear time that was correctly classified by the algorithm in each participant ranged from 67.0 to $98.8 \%$, with a mean of $90.5 \pm 11.9 \%$. In three participan ts the algorithm overestimated non-wear; in the other participants the algorithm underestimated non-wear.

The sensitivity and specificity of the $20 \mathrm{~min}$ algorithm were respectively $86.2 \%$ and $83.3 \%$. In one participant, the CAM provided data on only two axes which seemed to lead to extra false negatives and an over estimation of wear time. When we excluded the data of this participant, sensitivity increased to $92.0 \%$.

\section{Discussion}

Results of the current study showed that a time window of 20 min yielded the most accurate estimates of wear and non-wear when applying an algorithm on raw tri-axial data collected from overweight people. Commonly used algorithms have a time window of 60 min, allow for I to 2 min with counts up to the sedentary cut off point, and utilise the counts on the vertical axis only $(90,93)$. Recent studies have recommended time windows of $90-120 \mathrm{~min}(69,103)$ to reduce the amount of false positives (i.e. misclassification of wear as non-wear). However, in our measurements, a substantial proportion of the nonwear intervals were shorter than an hour. This increases the risk of false negatives, i.e. non-wear misclassified as wear (98). Therefore, both measurement states (wear and nonwear) should be considered and analysed when choosing and optimizing non-wear algorithms (98). While studies have compared the total non-wear time of the algorithm with the comparator and might have found acceptable agreement $(69,94,103)$, it is essential that the time excluded from analysis corresponds precisely to the actual nonwear. Therefore, validation studies should include analyses of starting and ending times of each interval to decrease the risk of excluding wear time or including non-wear time in analyses. In the current study, we calculated both the sensitivity and specificity of the algorithm to assess whether each wear and non-wear interval was classified correctly. The time window of 20 min led to an acceptable sensitivity and specificity in both the optimisation analysis $(79.1 \%$ and $86.4 \%$ respectively) and the validation $(86.2 \%$ and $83.3 \%$ respectively). Although a larger time window showed higher specificity (i.e. less non-wear misclassified as wear) and a shorter time window showed higher sensitivity, these time windows were not chosen due to the related decreases in sensitivity or specificity. Low sensitivity would lead to incorrect analysis of data, possibly leading to overestimation of sedentary time; in contrast, low specificity would lead to wrongly excluded data, possibly leading to underestimation of sedentary time. Both situations are undesirable, and the 
combination of sensitivity and specificity values led to the decision to adopt a 20 min time window.

Previous studies often used uni-axial and pre-processed data, providing less information when compared to our tri-axial device which provided the raw data. However, one other study comparing algorithms in a tri-axial activity monitor found results similar to the studies using uni-axial data (69). Moreover, the utilisation of tri-axial data improved nonwear detection in the wrist-worn monitor (69). We did not compare uni- with tri-axial in the current study; therefore, we cannot conclude whether the use of three axes is beneficial over one axis. As far as we know, this is the first study using raw and I s epoch data. Most activity monitors are provided with a software package, offering pre-defined filtering and calculations of counts, often summarised in minutes. Data is smoothened when using I min epochs, leading to a certain loss of information (68). Smoothing does not only lead to altered estimations of physical activity and sedentary time, but also relates to the classification of wear and non-wear. Our raw data was summarised to I s epochs before the algorithm was applied, requiring less smoothing and providing a more accurate reflection of actual behaviour. It is probable that the small movements during sedentary time are detectable in CAM data, but not in the pre-processed data of other activity monitors. Therefore, the risk of misclassifying sedentary time as non-wear with a short time window could be decreased in our data, despite the fact that a longer time window has been hypothesised to be more valid in a sedentary population $(69,103)$. Although our algorithm showed lower specificity than that reported by Winkler et al. (20I2), it is still considered acceptable for utilisation in physical behaviour analyses.

The role of placement of activity monitors in relation to the accuracy of non-wear algorithms should be further explored. The commonly used algorithm with a 60 min time window has mainly been applied to waist-worn activity monitors $(90,93)$. Choi et al. (2012) also assessed non-wear algorithms in a wrist-worn activity monitor. Although the 90 min time window was found to be optimal for both wearing locations (waist and wrist), the wrist location showed less bias in wear time classification (69). Wrist-worn activity monitors seem to enhance compliance; however, the classification of sedentary time is challenging (104). The CAM is thigh-worn to enable discrimination of sedentary time from standing and active time $(8 \mathrm{I}, \mathrm{I} 02)$. One could argue that an activity monitor placed on an extremity (arm or leg) would be more sensitive to small movements during sedentary time, decreasing the risk of incorrectly classified non-wear (105, 106). However, in contrast to our findings in the thigh-worn monitor, the study concerning non-wear of the wrist-worn monitor indicated that a 90 min time window would be optimal (69). A possible explanation for this discrepancy is the low amount of non-wear time in the study 
of Choi et al (20I2). In addition, the fact that the wrist-worn device was water-proof might have decreased the probability of short non-wear periods. Unfortunately, no information was available about the duration of non-wear periods in that particular study. Therefore, although current findings indicate possibilities for the utilisation of tri-axial accelerations for the identification of non-wear, research in activity monitors with other placement is required.

One strength of our study is the validation of the optimised non-wear algorithm. Optimisation of an algorithm is the first step towards automated non-wear detection; validation of this algorithm in other participants is the second and often overlooked step. Only after validation can researchers make solid conclusions about algorithm accuracy. In addition, we defined our algorithm specifically for our target population: overweight adults. As the accuracy of non-wear algorithms may differ due to participant characteristics (9I), it is essential to validate the algorithm in the population it will be used for.

One limitation of our study is that the results apply to the CAM specifically; however, raw and I s epoch data can be collected with other devices as well, and conclusions about the short time window might be transferrable. Although we used data from our target population, data from only sixteen participants was used. In addition, we were limited to using diaries as the criterion measure for wear and non-wear time. Nevertheless, we checked all wear and non-wear intervals in the graphical representations of the raw data, to eliminate wrongly registered intervals.

\section{Conclusion}

Based on the current optimisation and validity study we conclude that the algorithm with a time window of $20 \mathrm{~min}$ is acceptably sensitive and specific for participants who are overweight. The characteristics of algorithms differ between populations, devices, device placement and data-processing, and should therefore be tested thoroughly in the population of interest. 


\section{CHAPTER 4}

Effectiveness and cost-effectiveness of 'BeweegKuur', a combined lifestyle intervention in the Netherlands: rationale, design and methods of a randomized controlled trial

Brenda AJ Berendsen Marike RC Hendriks Evert ALM Verhagen Nicolaas C Schaper Stef PJ Kremers Hans HCM Savelberg 


\begin{abstract}
Background Improving the lifestyle of overweight and obese adults is of increasing interest in view of its role in several chronic diseases. Interventions aiming at overweight or weight-related chronic diseases suffer from high drop-out rates. It has been suggested that Motivational Interviewing and more frequent and more patient-specific coaching could decrease the drop-out rate. BeweegKuur is a multidisciplinary lifestyle intervention which offers three programmes for overweight persons. The effectiveness and the costeffectiveness of intensively guided programmes, such as the supervised exercise programme of BeweegKuur, for patients with high weight-related health risk, remain to be assessed. Our randomised controlled trial compares the expenses and effects of the supervised exercise programme with those of the less intensively supervised start-up exercise programme.
\end{abstract}

Methods The one-year intervention period involves coaching by a lifestyle advisor, a physiotherapist and a dietician, coordinated by general practitioners (GPs). The participating GP practices have been allocated to the interventions, which differ only in terms of the amount of coaching offered by the physiotherapist. Whereas the start-up exercise programme includes several consultations with physiotherapists to identify barriers hampering independent exercising, the supervised exercise programme includes more sessions with a physiotherapist, involving exercise under supervision. The main goal is transfer to local exercise facilities. The main outcome of the study will be the participants' physical activity at the end of the one-year intervention period and after one year of follow-up. Secondary outcomes are dietary habits, health risk, physical fitness and functional capacity. The economic evaluation will consist of a cost-effectiveness analysis and a cost-utility analysis. The primary outcome measures for the economic evaluation will be the physical activity and the number of quality-adjusted life years. Costs will be assessed from a societal perspective with a time horizon of two years. Additionally, a process evaluation will be used to evaluate the performance of the intervention and the participants' evaluation of the intervention.

Discussion This study is expected to provide information regarding the additional costs and effects of the supervised exercise programme in adults with very high weight-related health risk.

Trial registration Current Controlled Trials ISRCTN46574304 


\section{Background}

The increasing prevalence of overweight and obesity is a major problem in Western countries. People who are overweight are at higher risk of developing type 2 diabetes mellitus, cardiovascular disease and certain types of cancer (107). In addition, their healthrelated quality of life decreases due to the overweight as such as well as to related comorbidities (108). In the Netherlands, $42 \%$ of women and $53 \%$ of men are overweight $\left(\mathrm{BMI}>25 \mathrm{~kg} / \mathrm{m}^{2}\right)$, of which $\mathrm{I} 2 \%$ and $\mathrm{II} \%$ respectively are obese $\left(\mathrm{BMI}>30 \mathrm{~kg} / \mathrm{m}^{2}\right)(\mathrm{I} 09)$. Health care expenses caused by overweight in the Netherlands amounted to half a billion Euros in 1999 (110).

Not only overweight but also physical inactivity have been associated with chronic diseases like type 2 diabetes and cardiovascular disease (I I I-I I3). Intervening in people's lifestyles could help decrease the severity of chronic diseases and the risk of developing them. Combined lifestyle interventions aimed at increasing physical activity and improving dietary behaviour have been shown to have positive effects on metabolic and cardiovascular risk factors (e.g. weight, waist circumference, fat mass, HDL-cholesterol and triglyceride values and blood pressure) in persons at risk for developing chronic diseases $(3 \mathrm{I}, 32,35,5 \mathrm{I}, \mathrm{II} 4, \mathrm{II})$, as well as in patients who have already developed type 2 diabetes $(63,1 / 6-I \mid 8)$. Beneficial effects are still evident after a follow-up period of several years $(5 \mathrm{I}, \mathrm{I} \mid 3)$.

Recently, a multidisciplinary combined lifestyle intervention for type 2 diabetes patients, called BeweegKuur, has been developed by the Netherlands Institute for Sport and Physical Activity (NISB) $(56,57)$. Its target population currently also comprises overweight and obese patients. The primary goal of the BeweegKuur interventions is to improve physical activity and dietary behaviour and thereby decrease health risks. A recent study reported, however, that the adherence to exercise intervention programmes varies widely, from $10 \%$ to $80 \%$ (119). The main causes of drop-out are exercise-related injuries and motivational factors (I I6). It seems likely, therefore, that the use of Motivational Interviewing $(119,120)$ and the individualisation of the BeweegKuur programmes would result in lower drop-out rates. In addition, it has been proposed to have practice nurses play a key role in the adoption of long-term behavioural change by providing this individualised guidance in the primary health care setting $(32,50)$. In the BeweegKuur programmes, the participant's behavioural change is supported by a team consisting of a general practitioner (GP), a lifestyle advisor (LSA), a physiotherapist and a dietician. The LSA (who may be a practice nurse or a physiotherapist) has the key role in this multidisciplinary team and offers wide-ranging lifestyle counselling aimed at promoting physical activity, improving diet and reducing psychological barriers by means of 
Motivational Interviewing (120). A physiotherapist provides coaching for physical activity to enable participants to transfer to local exercise facilities, and a dietician provides advice on dietary improvement. The use of the BeweegKuur programmes in primary care has proved to be feasible, as health care providers as well as participants are very positive about the programmes after having implemented it (I2I, I22).

Care providers using BeweegKuur offer three programmes, differing in the amount of support. The independent exercise programme is intended for overweight and obese individuals without comorbidities, while the start-up exercise programme and the supervised exercise programme are both intended for adults with overweight or obesity who suffer from comorbidities or are morbidly obese. An earlier study showed that the amount of support required to achieve lifestyle changes increases with the severity of overweight and the presence of comorbidities (32). Additionally, the number of feedback sessions is believed to be positively related to programme adherence (II9). Hence, the supervised exercise programme involves more coaching by the physiotherapist.

Less intensively supervised programmes have been shown to be effective and costeffective for people with type 2 diabetes or an increased risk of developing type 2 diabetes (5I, 63, II5). The effectiveness and cost-effectiveness of intensively supervised programmes for a population with very high weight-related health risk remain to be studied. Therefore, our randomised controlled trial aims to evaluate the effects of the supervised exercise programme, in terms of the amount of physical activity and related health risks, and its cost-effectiveness, compared to those of the start-up exercise programme, for this population. The time horizon of the study will be two years. The economic evaluation will involve cost-effectiveness and cost-utility analyses from a societal perspective. In addition, a process evaluation is planned.

\section{Methods}

Study design

The present study is a clustered, multi-centre, randomised controlled trial evaluating the effectiveness and cost-effectiveness of the supervised exercise programme' versus the less intensively supervised start-up exercise programme' for patients with very high weightrelated health risk. Thirty Dutch GP practices, each collaborating with a practice nurse, a physiotherapist and a dietician, have been randomly assigned to the control or experimental condition. In experimental practices, participants will take part in the supervised exercise programme', while participants in a control practice will take part in the start-up exercise programme'. Clinical outcome measurements take place at baseline, 
after 12 months (the end of the intervention period) and after 24 months (Figure 4.I). In addition, self-administered questionnaires comprising cost-, effect- and process-related outcome measures will be sent to the participants every three months.

This study is approved by the Medical Ethics Committee of the Maastricht University Medical Centre and is registered with Current Controlled Trials (ISRCTN46574304). The study is funded by The Netherlands Organization for Health Research and Development (ZonMW; project number: 123000002).

\section{Randomisation}

To reduce the risk of contamination between participants, and the risk of bias at the level of the professionals involved, entire practices have been allocated to the control or experimental condition. Prior to randomisation, all practices have been matched pair wise based on size and location in an urban or rural area, to create two equivalent samples of I5 practices. In each pair, one practice has been randomised to the control condition, while the other was randomised to the experimental condition. To reduce the risk of contamination within a region, practices in the same region were allocated to the same condition as the first practice in that region that was randomised.

\section{Participants}

Inclusion of participants started in July 2010. Inclusion criteria are (I) being overweight or obese (BMI 25-35 kg/m²) combined with the following serious related comorbidities: sleep apnoea, arthritis, cardiovascular disease and/or type 2 diabetes; or (2) being morbidly obese (BMI $35-40 \mathrm{~kg} / \mathrm{m}^{2}$ ) but without these related serious comorbidities. In addition, participants should currently fail to meet the Dutch norm for healthy physical activity (30 minutes of moderate to vigorous physical activity on at least 5 days a week), have to be sufficiently motivated to change their physical activity level and dietary behaviour (to be judged subjectively by the LSA during intake) and have to give their informed consent. Participants are being included via GPs, practice nurses and physiotherapists. The GP, practice nurse or physiotherapist selects patients by discussing the intervention during a consultation. However, they can also recruit patients actively (e.g. by searching the health care provider's records). The LSA screens the patients for eligibility. Exclusion criteria are serious mobility limitations precluding participation in the intervention programme, such as severe cardiac failure, serious angina pectoris and rheumatoid arthritis. Pregnancy is also an exclusion criterion. The GP decides whether patients should be excluded. 


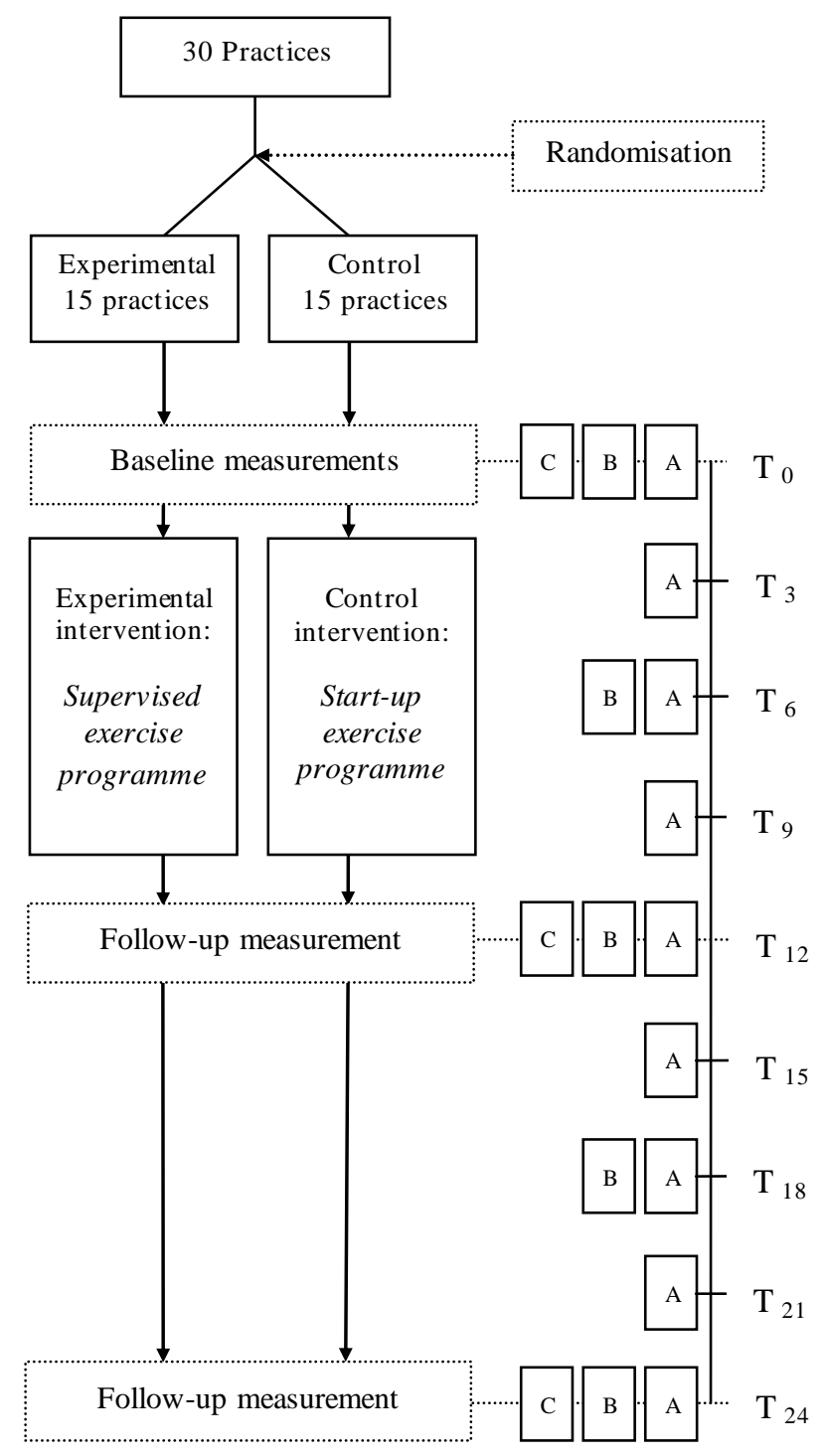

Figure 4.I. Flow chart of the BeweegKuur randomised controlled trial and measurements.

A. Health care utilisation and productivity losses, EQ5-D and process items in questionnaire every three months; B. Clinical outcomes measured in questionnaire every six months; C. Clinical outcome measurements performed every year. Table 4.2 shows outcomes in each category.

T0-T24 represent moments of measurement. $\mathrm{T} 0=$ Baseline; $\mathrm{T} 3=$ Three months after baseline; $\mathrm{T} 6=$ Six months after baseline; ...; T24 = 24 months after baseline. 


\section{Blinding}

Although it is not possible to blind the professionals, randomisation at the level of GP practices decreases the risk of contamination among the professionals. Participants are not aware of the allocation of their practice to the experimental or control condition.

To identify effects of observer bias, clinical measurements that might be affected (Åstrand test, Valk neuropathy test, body composition, hand grip strength and Timed Up and Go Test) will be repeated by a researcher blinded for the randomisation of the practices and the baseline characteristics of the participants. Repeated measurements will be done for 20 participants in five randomly chosen control and five randomly chosen experimental practices.

\section{Interventions}

After inclusion, participants have several consultations with the LSA, dietician and physiotherapist during a one-year intervention period. The number of consultations differs per programme (table 4.I).

Table 4.I. Contents and number of meetings planned in the control and experimental interventions

\begin{tabular}{|c|c|c|}
\hline & $\begin{array}{l}\text { Experimental intervention } \\
\text { the supervised programme }\end{array}$ & $\begin{array}{l}\text { Control intervention } \\
\text { the start-up programme }\end{array}$ \\
\hline Contents of LSA meetings & No. of meetings & No. of meetings \\
\hline Intake & 1 & I \\
\hline Guidance and follow-up & 5 & 5 \\
\hline $\begin{array}{l}\text { Contents of dietician } \\
\text { meetings }\end{array}$ & No. of meetings & No. of meetings \\
\hline Intake & 1 & I \\
\hline \multicolumn{3}{|l|}{ Guidance and follow-up } \\
\hline - Individual & 2 & 2 \\
\hline - Group & 7 & 7 \\
\hline $\begin{array}{l}\text { Contents of physiotherapist } \\
\text { meetings }\end{array}$ & No. of meetings & No. of meetings \\
\hline Intake & $\mathrm{I}$ & I \\
\hline Setting up exercise plan & 2 & I \\
\hline Supervised exercise & $26-34$ & - \\
\hline Follow-up & $3-4$ & 4 \\
\hline
\end{tabular}




\section{Experimental intervention (supervised exercise programme)}

The LSA has a key role in supporting the participants and is the point of contact between the participants and the other health care providers in the BeweegKuur programme. Following an individual intake to set personal goals, participants will have five in dividual consultations with the LSA to discuss progress in terms of behavioural change, roughly once every 10 weeks, during the one-year intervention period. Consultations with the dietician will consist of nutritional recommendations, education, coping with high-risk situations, checking dietary behaviour and fellow-sufferer contact. Advice will be based on various Dutch guidelines for diabetes, overweight and obesity (123-125). After an individual intake session by the dietician, seven group sessions are planned. The group meetings comprise guidance and advice by the dietician and are scheduled throughout the year. In addition, two individual follow-up meetings are planned during the intervention period to prevent relapse.

The physiotherapist will provide coaching to enable participants to exercise unsupervised in local exercise facilities. Coaching by the physiotherapist will be initiated by setting out personal goals and identifying barriers hampering engagement in physical activity. Coaching will consist of supervised exercise to overcome any barriers identified and increase physical capacity. Two or three sessions of supervised exercise per week will be planned over a period of 12 weeks. After these 12 weeks, the physiotherapist will evaluate whether the participant is able to exercise without supervision. The coaching period can be extended by four weeks if the participant does not seem able to exercise independently in local facilities. In all, the physiotherapist's coaching will take 12 to 16 weeks. After coaching by the physiotherapist has ended, the five follow-up consultations with the LSA and three or four follow-up consultations with the physiotherapist are planned, to help participants adopt and continue independent exercise activities. Both the LSA and the physiotherapist will help the participant find suitable existing exercise facilities during the entire intervention period.

\section{Control intervention (start-up exercise programme)}

The number of consultations and the characteristics of the guidance provided by the LSA and the dietician in the control condition are the same as in the experimental condition. However, participants in the start-up exercise programme will only have six consultations with the physiotherapist, which are planned during the first two months of the intervention period. The consultations with the physiotherapist consist of identifying barriers to physical activity and drawing up a plan to remain physically active without supervision by health care providers. If deemed necessary by the physiotherapist, 
participants can exercise under supervision during these meetings to overcome barriers to physical activity. Progress and complications relating to the exercise plan will be discussed in consultations with the physiotherapist from approximately two months after the start of the intervention. Additionally, participants will be coached in the adoption and maintenance of independent exercise activities during the five follow-up consultations with the LSA during and after the two months of guidance by the physiotherapist.

\section{Outcomes}

Clinical assessments will be done by the BeweegKuur health care providers (LSA or physiotherapist) in their own practice. After the baseline measurement, three different measurement intervals will be used, depending on the variable to be measured: $(A) 3$ months, (B) 6 months and (C) 12 months (figure 4.I and table 4.2).

Table 4.2. Type of outcomes in each measurement category (time intervals are shown in figure 4.I)

\begin{tabular}{|c|c|c|c|c|c|}
\hline A. & $\begin{array}{l}\text { Costs, utility and process } \\
\text { assessment (self- } \\
\text { administered } \\
\text { questionnaires) }\end{array}$ & & $\begin{array}{l}\text { Clinical outcomes } \\
\text { (self-administered } \\
\text { questionnaires) }\end{array}$ & C. & $\begin{array}{l}\text { Clinical outcomes } \\
\text { (measurements } \\
\text { by professionals) }\end{array}$ \\
\hline $\begin{array}{l}- \\
- \\
-\end{array}$ & $\begin{array}{l}\text { Health care use, other } \\
\text { expenses and productivity } \\
\text { losses } \\
\text { Quality of life } \\
\text { Process evaluation }\end{array}$ & & $\begin{array}{l}\text { Physical activity } \\
\text { Dietary behaviour }\end{array}$ & $\begin{array}{l}- \\
- \\
-\end{array}$ & $\begin{array}{l}\text { Physical activity by } \\
\text { accelerometry } \\
\text { Risk factors for } \\
\text { comorbidities } \\
\text { Physical fitness }\end{array}$ \\
\hline
\end{tabular}

\section{Physical activity}

The primary outcome of this study will be the amount of physical activity that participants engage in, as measured by means of accelerometry and the short version of the International Physical Activity Questionnaire (IPAQ). The IPAQ short form will be included in the participants' questionnaire every six months, and consists of questions concerning the time spent on physical activity at specific intensities and the number of days on which this happened. Median values of activity categories will be calculated and expressed as metabolic equivalent (MET) minutes per week. The self-administered IPAQ short form has been reported to be sufficiently valid and reliable for use in developed countries (126).

Accelerometry offers an objective way to assess physical activity. The CAM is a tri-axial accelerometer developed and manufactured by Maastricht Instruments. The CAM software is able to distinguish between sedentary behaviour, standing and activity, and has 
been validated for adults in laboratory conditions (8I). The device weighs approximately $100 \mathrm{~g}(63 \times 45 \times 18 \mathrm{~mm})$ and its sampling frequency is $25 \mathrm{~Hz}$. Participants will wear the CAM for four consecutive days; data from waking up until going to sleep will be used for analysis. Because the CAM is not waterproof, participants will have to remove the CAM for swimming, showering, bathing etc. and will be asked to write down their activities in a diary for such non-wearing intervals. The main outcomes of the CAM measurements will be the amount of moderate to vigorous physical activity (MVPA) and the amount of time spent sedentary, standing or active.

\section{Dietary habits}

The short version of the Fat Food questionnaire will be included in the questionnaire sent to the participants every six months (127). The length of this validated Fat Food questionnaire has been reduced to maximise the number of questions completed. Twentyone items address the respondent's regular eating pattern (e.g. consumption of vegetables, lettuce and fruit) and consumption of high-fat meals (e.g. take-away food), snacks and candy.

\section{Risk factors for comorbidities}

Body composition (weight, fat mass and fat-free mass) will be measured with a tetra polar bioelectrical impedance device (OMRON BF5II). Blood samples will be taken to analyse fasting glucose (mmol/L), HbAlc (\% or $\mathrm{mmol} / \mathrm{L})$, total cholesterol ( $\mathrm{mmol} / \mathrm{L})$, HDL $(\mathrm{mmol} / \mathrm{L})$, triglycerides $(\mathrm{mmol} / \mathrm{L})$ and creatinine $(\mu \mathrm{mol} / \mathrm{L})$. Systolic and diastolic blood pressure, resting heart rate and $\mathrm{BMI}$ will also be measured.

\section{Physical fitness}

Peripheral neuropathy is related to functional capacity in type 2 diabetes patients (I28). Diabetic neuropathy will be examined using the Valk neuropathy test (I29), while hand grip strength $(130,13 \mid)$ and the timed 'up and go' test will be used as measures of functional capacity (132). To familiarise participants with the Borg scale, it will be used during the timed 'up and go' and hand grip strength tests.

The participant's aerobic capacity will be estimated using the sub-maximal Åstrand cycle test (133, 134). The Åstrand cycle test will always be administered by the physiotherapists at their own practice. Participants will start cycling at 50 Watt at a pedal rate of 50 revolutions per minute. The test will be conducted at heart rates between 120 and maximum heart rate. The heart rates of the fifth and sixth minutes of the test will be recorded and used to estimate the aerobic capacity from a nomogram (I33). This 
estimated aerobic capacity will be corrected for age (135). In addition, the rate of perceived exertion will be recorded at each work level by means of the Borg score, with a range of 6-20. Participants whose heart rate cannot be used as a reference for physical fitness (e.g. patients who use beta blockers) will do the Åstrand cycle test with a Borg score between 13 and 17 (136).

\section{Economic evaluation}

The economic evaluation will compare costs and effects of the supervised exercise programme with those of the start-up exercise programme. The economic evaluation will be performed from a societal perspective, which implies that all relevant costs and outcomes will be taken into account, regardless of who pays the costs and who benefits from the effects. A time horizon of two years will be used.

Both a cost-effectiveness analysis (CEA) and a cost-utility analysis (CUA) will be done. The CEA will present clinical outcomes in terms of physical activity measured by means of accelerometry and the short version of the IPAQ (126). The CUA will present effects in terms of quality-adjusted life years (QALYs) measured by means of the internationally developed EuroQol (137) in three-monthly self-administered questionnaires. The QALY incorporates multiple aspects of the intervention (e.g. side-effects) and allows comparisons among different (lifestyle) interventions in different target populations. A direct value for each state of health will be generated using social tariff, which involves an algorithm for interpolating EuroQol outcomes into population utilities based on the United Kingdom valuation (138) and the Dutch valuation (139).

Programme costs, health care costs, patient and family costs as well as loss of productivity will be assessed. Volumes of healthcare use, loss of productivity and other expenses will be identified by means of three-monthly self-administered questionnaires. Cost valuation will use the Dutch manual for cost analysis in health care research (140), while real costs will be used otherwise. Cost prices will be expressed in Euros from the baseline year $20 \mathrm{II}$, and otherwise indexed to the baseline year, as suggested in the Dutch manual (I4I). Because the recruitment period will be 12 months and the follow-up period 24 months, costs and effects in the second year of follow-up will be discounted.

\section{Process evaluation}

A process evaluation will be used to gain insight into reach and the attendance rates of the target population, implementation fidelity, delivered intervention dose, and participant perception of the intervention (I42) in order to support the interpretation of the effects. The process evaluation will assess personal factors of participants (e.g. self-efficacy, 
motivation towards being physically active and eating healthy), self-report environmental variables as used in the International Physical Activity Prevalence Study (e.g. presence of pavements and perceived neighbourhood safety) (143), number and duration of the consultations with the health care providers involved, satisfaction with the intervention contents and feasibility of the intervention. The process will be evaluated by means of selfadministered questionnaires for participants, with closed and open-ended questions. In addition, registration forms, short surveys and semi-structured interviews with the relevant professionals in each practice will assess relevant barriers and facilitators for intervention implementation. Adverse events will be recorded.

\section{Sample size}

The intended sample size is based on the amount of MVPA in minutes per week. An increase of 50 minutes of MVPA per week by participants in the supervised exercise programme, as compared to participants in the start-up exercise programme will be considered a clinically meaningful increase in MVPA. The standard deviation of MVPA in this population has been reported to be 120 minutes/week (I44). A sample size of 9 I participants per condition will be needed to detect a difference of 50 minutes of MVPA per week, with $80 \%$ power and $5 \%$ significance (two-sided). Assuming a drop-out rate of $30 \%$, this would require 119 participants in each programme, i.e. 238 participants in total. Allocation to the conditions, however, will take place at the level of GP practices, so clustering of patients within these practices should be taken into account. Assuming an intra-cluster correlation of $5 \%$, and a total of 20 participants per practice, a total sample of 24 practices $(n=480)$ will be needed. As practices may also drop out of this study, we will include and allocate an additional six practices to account for this potential drop-out. The choice of six practices is completely arbitrary. This results in a projected total sample of 600 participants divided over 30 practices.

\section{Analysis}

Baseline characteristics (BMI, age, gender, amount of MVPA by accelerometry) of both participants and clusters will be analysed by means of descriptive statistics. Statistical analyses will be performed according to the intention-to-treat principle, while additional analyses will be done using the per-protocol principle.

Differences between outcomes in the control and intervention groups at different time points will be assessed using multi-level analyses. This type of analysis takes into account the longitudinal nature of the data, as well as the impact of cluster randomisation. 
Differences in costs and effects will be presented as incremental cost-effectiveness ratios (ICERs). ICERs represent the differences in mean costs between the experimental and control interventions in the numerator and the difference in mean effects between the two groups in the denominator. Sensitivity analysis will be used to assess the robustness of the assumptions made in our base case analysis.

Outcomes of the process evaluation will be studied by means of descriptive statistics.

\section{Discussion}

The aim of this study is to determine whether the supervised exercise programme of the BeweegKuur intervention has positive effects on physical activity levels compared to the less intensively supervised start-up exercise programme in a population of overweight and obese adults with very high weight-related health risk, and to assess the difference in costs involved between the two lifestyle programmes. The risk of chronic diseases is known to decrease if overweight or obese persons achieve a more physically active lifestyle. This might also reduce health care expenses. Therefore, an improved lifestyle resulting from an intervention like BeweegKuur is expected to have major positive consequences at both individual and societal level.

Increasing adherence to lifestyle interventions is crucial. The proposed solutions (Motivational Interviewing and patient-specific guidance) might decrease the drop-out rate, thereby increasing the possible effects of the intervention and decreasing health care expenses. Nevertheless, these solutions require extra time investment by health care providers, raising intervention costs, so examining the cost-effectiveness of lifestyle interventions in primary care is of great social interest. The economic evaluation will provide insight into the cost-effectiveness regarding the effects on quality of life and physical activity, to support decisions concerning insurance coverage of the BeweegKuur intervention and similar lifestyle interventions.

Objectively measuring physical activity levels enables accurate conclusions to be drawn about the direct effects of the intervention. Moreover, this will afford new insights into physical activity and inactivity patterns in an overweight population with very high weightrelated health risk.

This study aims to gain insight into the cost-effectiveness of the supervised exercise programme compared to the start-up exercise programme, in order to inform decision and policy makers about the implementation of BeweegKuur in primary care in the Netherlands. In addition, the process evaluation will provide detailed information about the feasibility of implementing these two interventions and the degree of satisfaction of 
participants, and will also provide some insight into the mechanisms by which the components of the intervention exert their effects. 


\section{CHAPTER 5}

The implementation and sustainability of a combined lifestyle intervention in primary care: mixed method process evaluation

Brenda AJ Berendsen

Stef PJ Kremers

Hans HCM Savelberg

Nicolaas C Schaper

Marike RC Hendriks 


\section{Abstract}

Introduction The impact of physical inactivity and unhealthy diet on health is increasingly profound. Lifestyle interventions targeting both behaviours simultaneously might decrease the prevalence of overweight and comorbidities. The Dutch BeweegKuur is a combined lifestyle intervention (CLI) in primary care, to improve physical activity and dietary behaviour in overweight people. In a cluster randomised controlled trial, the (cost)effectiveness of an intensively guided program has been compared to a less intensively guided programme. This process evaluation aimed to assess protocol adherence and potential differences between clusters. In addition, sustainability (i.e. continuation of the $\mathrm{CLI}$ in practice after study termination) was evaluated.

Methods Existing frameworks were combined to design the process evaluation for our intervention and setting specifically. We assessed reach, fidelity, dose delivered and received, context and implementation strategy. Both qualitative and quantitative data were used for a comprehensive evaluation. Data were collected in semi-structured interviews with health care providers $(\mathrm{HCPs}, \mathrm{n}=25)$, drop-out registration by $\mathrm{HCPs}$, regular questionnaires among participants $(n=4 I I)$ and logbooks kept by researchers during the trial.

Results Protocol adherence by professionals and participants varied between the programmes and clusters. In both programmes the number of meetings with all HCPs was lower than planned in the protocol. Participants in the supervised programme attended, compared to participants in the start-up programme, more meetings with physiotherapists, but fewer with lifestyle advisors and dieticians. The BeweegKuur was not sustained, but intervention aspects, networks and experiences were still utilised after finalisation of the project. Whether clusters continued to offer a CLI seemed dependent on funding opportunities and collaborations.

Conclusions Protocol adherence in a CLI was problematic in both HCPs and participants. Mainly the amount of dietary guidance was lower than planned, and decreased with increasing guidance by physiotherapist. Thus, feasibility of changing physical activity and dietary habits simultaneously by one intervention in one year was not as high as expected. Also the sustainability of CLI was poor. When a CLI programme is started, re-invention should be allowed and maximum effort should be taken to guarantee long-term continuation, by planning both implementation and sustainability carefully. 


\section{Introduction}

Obesity, physical inactivity and unhealthy diet have a combined and independent impact on health $(11,27,111,113)$ with increasing social and economic burden. In 2010, overweight related health care costs reached up to 1.6 billion euros in the Netherlands (4). Accordingly, much effort has been put into promoting healthy lifestyles, resulting in programmes ranging from medical treatment to preventive lifestyle interventions.

In general, several studies suggest that combined lifestyle interventions (CLI) aimed at the overweight and obese population yield positive results $(5 \mathrm{I}, 145,146)$. Unfortunately, such interventions often suffer from high drop-out rates, mainly due to exercise injuries and motivational factors $(1 / 6,119)$. In addition, studies often lack implementation in real world setting $(3 \mathrm{I}, 35)$, limiting the generalisability of results to daily practice. Furthermore, sustainability (i.e. continuation in practice after study termination) of lifestyle interventions is crucial to provoke effects on public health. The BeweegKuur is a CLI offered by a multidisciplinary team of health care providers (HCPs) in primary care $(56,57)$ and aims at promoting and sustaining both physical activity and healthy diet to improve health of people who have overweight or obesity. In 2007 the BeweegKuur has been developed by the Netherlands Institute for Sport and Physical Activity (NISB), commissioned by the Dutch ministry of Health, Welfare and Sports. Over the years, the BeweegKuur has been adapted based on process evaluations and now comprises one year guidance by a lifestyle advisor (LSA), physiotherapist and dietician. The amount of guidance by the physiotherapist depends on weight related health risk, based on BMI and presence of comorbidities (see methods section). A programme with six meetings with physiotherapist (start-up programme) has already been proven effective (63), however, the hypothesised effects of additional guidance (supervised programme; 26-34 meetings with physiotherapist) remained to be shown. Therefore, the effectiveness and costeffectiveness of the supervised programme compared to the start-up programme has been subject of a clustered randomised controlled trial (cRCT) (IOI). Thirty primary care health care clusters (HCCs) in the Netherlands participated in the study and were randomly assigned to either the less intensive control programme (the start-up programme) or the experimental programme (the supervised programme).

The effectiveness and cost-effectiveness of interventions in primary care depend heavily on process aspects, such as context and delivery of the programme. Moreover, process factors may differ between HCPs and HCCs, possibly influencing costs and outcomes (147). Therefore, process evaluation of complex lifestyle interventions has been advocated, especially in cRCTs (147). Moreover, studying the process prior to (cost-) effectiveness evaluation ensures a full evaluation of all potential lessons to be learned, 
instead of a pursuit of explanations for the (cost-)effectiveness outcomes which might introduce interpretation bias (148). The current study combined parts of several existing theoretical frameworks $(142,147,149-151)$ to construct a comprehensive structure to evaluate the process of this CRCT of the BeweegKuur specifically. By constructing our framework based on existing, generally adopted frameworks, we ensure a full evaluation of the BeweegKuur study. In short, our framework consisted of the following concepts: reach and recruitment, fidelity, dose delivered, dose received, context, implementation strategy and sustainability.

The current study evaluated the process of implementation, execution and continuation of the BeweegKuur in primary care from both participant and HCP perspective. We aimed to provide insight into possible barriers and facilitators in execution and sustainability of CLls in primary care, by carrying out the process evaluation prior to the effect and economic evaluation. Furthermore, the process evaluation aimed to gain in depth information for interpretation of the effectiveness and cost-effectiveness evaluation.

\section{Methods}

Intervention \& setting

This study evaluated the process of implementation, execution and sustainability within a multi-centre, clustered randomised controlled trial (cRCT) aimed at the effectiveness and cost-effectiveness of two intensities of a combined lifestyle intervention programme: the BeweegKuur (IOI). The BeweegKuur is a one-year intervention developed by the Netherlands Institute for Sport and Physical Activity (NISB) and aims at adopting a sustained healthy lifestyle. The BeweegKuur consists of programmes that differ in intensity of supervision. In this $\mathrm{CRCT}$, the most intensive CLI programme has been compared with a less intensive programme; the latter has been argued to be both effective and costeffective (63). Eligible participants were (I) either overweight or obese (BMI $25-35 \mathrm{~kg} / \mathrm{m}^{2}$ ) with at least one of the following serious related comorbidities: sleep apnoea, arthritis, cardiovascular disease and/or type 2 diabetes; or (2) morbidly obese (BMI $35-40 \mathrm{~kg} / \mathrm{m}^{2}$ ) but without these related serious comorbidities.

Thirty Dutch primary care HCCs were selected by NISB, based on expressed willingness to participate. Each HCC was a collaboration of one or more GPs, LSAs, physiotherapists and dieticians who recruited and/or guided participants. HCCs were assigned at random to the supervised programme, or to the less intensive start-up programme. HCCs allocated to the start-up programme did not offer the supervised programme during the current study. Prior to the study, each HCC consented to recruit 20 participants. A detailed description of the intervention and the $C R C T$ is provided in an earlier publication 
(IOI). Both programmes comprised six individual meetings with LSA, three individual meetings with a dietician and seven dietary group meetings. In addition, the start-up programme consisted of six individual meetings with physiotherapist, in comparison, the supervised programme consisted of six to seven individual and 26-34 group meetings with physiotherapist. It has been hypothesised that the additional amount of guidance within the supervised programme increases the effects on physical activity, dietary behaviour and health in the population with high weight related health risk. The initial individual meetings with the HCPs were aimed at setting personal goals and identifying barriers to a healthy lifestyle by means of Motivational Interviewing (MI), which were the basis for the further meetings. The physiotherapist offered coaching and guidance specifically for physical activity to facilitate transfer to local exercise facilities. At the end of the intervention (I 2 months after start), the participant had a meeting with LSA to evaluate the lifestyle changes and conclude the intervention.

This study is approved by the Medical Ethics Committee of the Maastricht University Medical Centre and is registered with Current Controlled Trials (ISRCTN46574304).

\section{Data collection}

Process evaluation data were gathered from both HCPs and participants. HCPs of five start-up and five supervised HCCs were selected to participate in face to face, semistructured interviews. HCCs in both conditions were selected based on relative success of recruitment (low, middle and high recruitment rate), urbanisation (rural, municipality and city) and type of HCC (cooperation of geographically separate practices and primary health care under one roof). At the moment of the interviews, the one year intervention was concluded in all participants. Interviews were held with $25 \mathrm{HCPs}$, of which eight physiotherapists, seven dieticians (of which 2 by phone calls), seven practice nurses with the role of LSA, one dietician with the role of LSA and two physiotherapists with the role of LSA. Two dieticians were not available for the interviews due to personal or organisational reasons. Additionally, every three months, all participants $(n=4 \mathrm{II})$ received a questionnaire specifically developed for the current study, which contained items regarding the process. The baseline questionnaire was distributed by the HCP; subsequent questionnaires were distributed and collected via mail by the researchers. In addition, information about drop-outs, reasons for dropping out and loss to follow up were gathered from HCP registries. Moreover, data were extracted from logbooks of informal communication between the HCPs and the research team (registered calls, emails and visits to $\mathrm{HCC}$ ). 


\section{Research framework}

Data were collected and presented in a framework which was designed by combining concepts from existing frameworks. Firstly, the RE-AIM framework provided the dimensions reach, efficacy, adoption, implementation and maintenance to illustrate public health impact of an intervention (I50). These dimensions were complemented with key concepts from work by Steckler and Linnan (2002) and Saunders et al. (2005) regarding the evaluation of CLI specifically (fidelity, implementation, dose delivered and received, reach, recruitment and context). In addition, the implementation strategy (149) and clusters were studied to reveal working mechanisms in complex interventions (I47). The specific contents are further elaborated on per concept.

\section{Reach and recruitment}

Recruitment of clusters (the HCCs) (147) as well as participants were evaluated (142, 15I). Interviews with HCPs were aimed at the recruitment procedure (e.g. the HCPs responsible for recruitment and source of participants) and the representativeness of the study population. Participant recruitment was registered per month in all HCCs. HCP measured length and weight, waist circumference and recorded age and sex. HbAlc was assessed and further demographics (nationality, employment, education level and marital status) were retrieved from the participant questionnaires at baseline. Nationality was categorised into Dutch and non-Dutch; employment was categorised into paid work, unpaid work and studying or not working; and marital status was categorised into married, unmarried, cohabiting, divorced and widowed. Education was categorised into low, middle or high based on highest level of completed education. In addition, questionnaires contained items about the recruitment procedure and reasons to participate in the BeweegKuur (e.g. 'What were main reasons for you to participate in the BeweegKuur?').

\section{Fidelity}

Fidelity was defined as the execution of the intended characteristics of the intervention (142, I5I). The main question addressing fidelity was whether the intervention was implemented consistently with the underlying working mechanisms. MI is one of the main mechanisms of the BeweegKuur $(56,57,120)$, and therefore crucial in the fidelity assessment. In addition, setting goals or a plan is essential for lifestyle change. The application of $\mathrm{Ml}$ and goal setting was discussed in the interviews with the HCPs and also the participant filled in questions regarding goal setting (e.g. 'Did you set goals with the physiotherapist regarding physical activity?'). 
Dose delivered

Dose delivered described the degree of execution of the programme by LSA, physiotherapist and the dietician according to protocol $(142,151)$. The number, content and characteristics of meetings were discussed in the interviews with HCPs. The participants' questionnaire contained questions about the number of meetings with BeweegKuur HCPs every three months (e.g. 'How often did you have a meeting with the LSA in the past three months?') and whether planned activities were performed by the HCPs (e.g. 'Was the BeweegKuur guidance clearly concluded by your LSA?').

\section{Dose received}

Dose received was defined as participant satisfaction and perception of the programme that was delivered to them $(142,15 \mathrm{I})$. Attempted reduction of drop-out and reaction to potential drop-out was discussed in the interviews with the HCPs. In addition, number of drop-outs and reasons were discussed and retrieved from HCPs' own registration, if available. The participant questionnaire contained questions regarding satisfaction with the programme and guidance on a scale of $\mathrm{I}-\mathrm{I} 0$ ( $\mathrm{I} 0$ is best score).

\section{Context}

Within the context we assessed aspects of the environment with a potential influence on execution and sustainability of the intervention $(142,147,15 I)$. Interviews contained discussion about the hindering and promoting factors of continuation of the intervention in the HCC. Also, collaboration to promote participant outflow to exercise facilities were discussed.

\section{Implementation strategy}

The implementation of an intervention should be planned carefully to facilitate sustainability of change (149). Implementation was mainly organised by NISB through the Regional Support Structure for Primary Health Care (ROS) (57). We evaluated the presence of support by ROS and NISB in the implementation and continuation of the intervention in the interviews with HCPs. 


\section{Data analysis}

The interviews were recorded, and a researcher not being the interviewer wrote notes about the content and non-verbal communication. Interviews were transcribed ad verbatim with F4 audio-transcription software (Dr. Dresing \& Pehl GmbH, Hamburg, Germany) by a researcher not being the interviewer. Afterwards, transcriptions were read and approved by the interviewer and subsequently made anonymous. Transcriptions were analysed by means of NVIVO 2.0 (QSR International Pty. Ltd., Warrington, UK) by BB, $\mathrm{MH}$ and MS. A node tree was developed based on the study framework to categorise the quotes from the interviews into the specific concepts. The first interview was analysed with the node tree independently by $\mathrm{BB}$ and $\mathrm{MH}$ and in case of disagreement between the coding by the two researchers, the node tree was adjusted by deleting, adding or combining nodes. This resulted in a definitive node tree used for the coding of all transcriptions (figure 5.I). All codes in transcriptions were read and approved by a different researcher than the coder $(\mathrm{BB}$ or $\mathrm{MH})$. BB selected important information from coded transcripts and $\mathrm{MH}$ checked the selection of important information from coded transcripts. In case of disagreement, the issue was discussed with SK. Quotes are depicted in the results between quotation marks in italics.

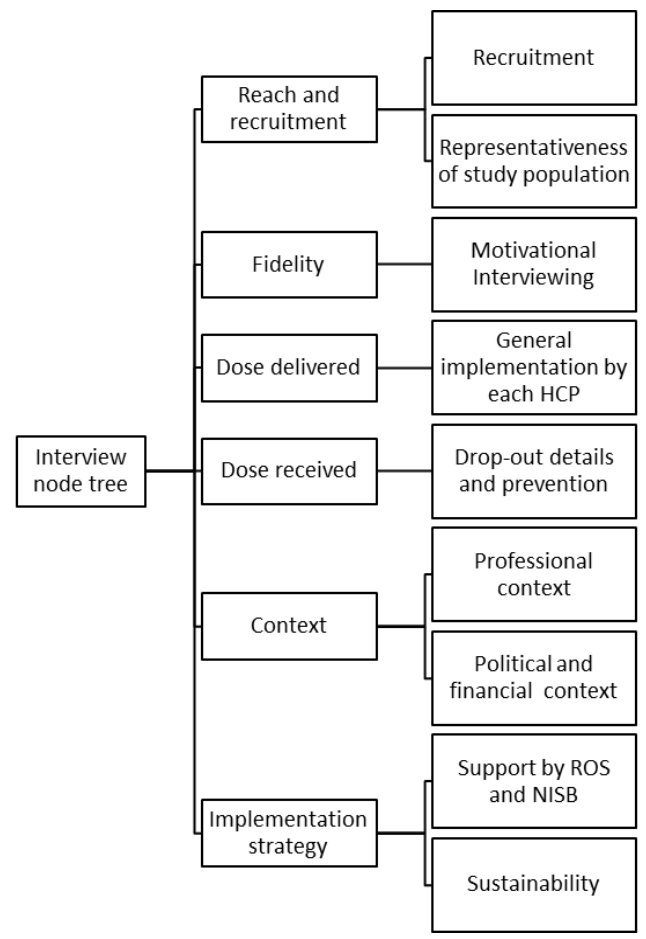

Figure 5.I. Node tree with interview contents 
Quantitative data were analysed in SPSS 21.0 with complete cases for the item of interest (ranging from 135 to 365 participants per analysis). Demographics and questionnaire data were depicted as mean \pm standard deviation and in percentages. Differences between the start-up and supervised condition were analysed with t-tests, Pearson chi square and Mann-Whitney $U$ tests. Differences between HCCs were analysed with one-way ANOVA or Kruskal-Wallis tests.

\section{Results}

Reach and recruitment

One start-up HCC dropped out before the start of the study for unknown reasons. One supervised HCC dropped out during the study due to organisational changes in the GP practice; this HCC failed to provide baseline measurements and did not perform any follow up measurement of the participants.

In total, 4II participants were recruited within 14 months, 247 participants in the supervised and 164 in the start-up programme, with two to 30 subjects per HCC. These numbers were lower than planned and the HCPs declared they had trouble finding suitable subjects, because many potential participants had already been asked to join in the past. In the supervised programme recruitment was higher, especially in the first four months (figure 5.2). Registries showed that start-up HCCs attributed their low recruitment rate to organisational changes in the HCP team and incorrect information from ROS regarding termination of recruitment. Supervised HCCs with low recruitment gave similar reasons. In addition, start-up HCCs had the possibility to offer the supervised programme prior to the study start.

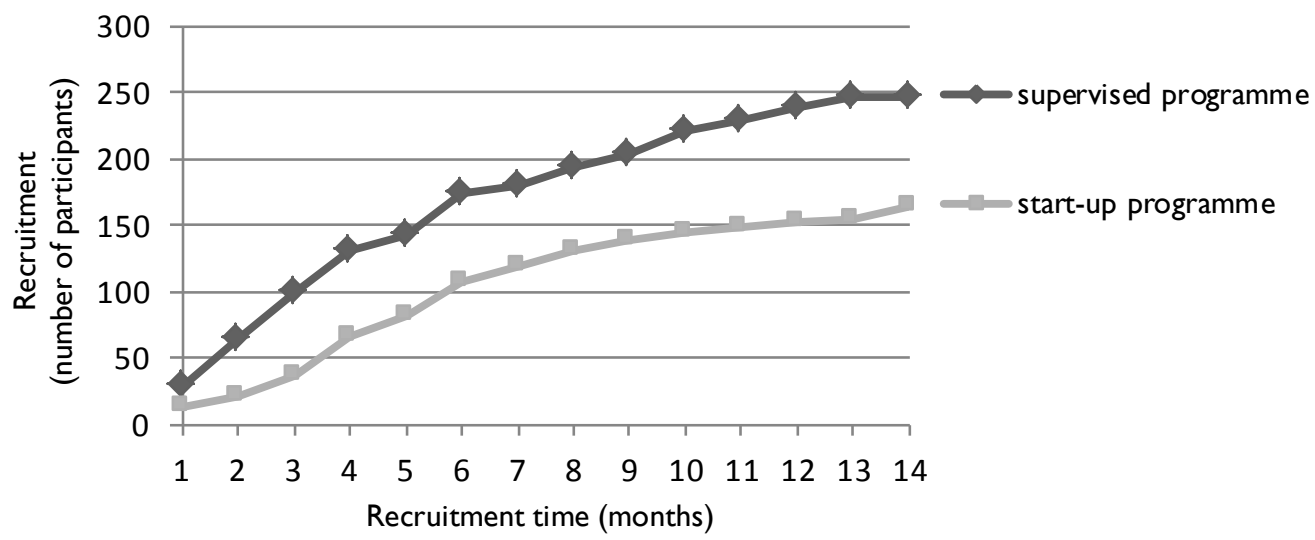

Figure 5.2. Cumulative recruitment numbers per month in the two research arms. 
Mean age of participants was 55.1 years $( \pm 12.4)$, the majority was female $(64.7 \%)$, with Dutch nationality (88.8\%) and married (65.6\%). Background characteristics did not differ between start-up and supervised participants, except for marital status ( $p=0.027$; table 5.I). Of all participants, $48.9 \%$ had diabetes type 2 at baseline, $30.2 \%$ did not have diabetes type 2, and of $20.9 \%$ presence of diabetes type 2 was unknown (no difference between programmes). Mean BMI of the participants was $34.5 \pm 4.4 \mathrm{~kg} / \mathrm{m}^{2}$, waist circumference II $3.2 \pm 11.2 \mathrm{~cm}$ and $\mathrm{HbAlc}$ level $6.37 \pm \mathrm{I} .12 \%$, with no differences between the two groups.

Baseline data revealed that $48.9 \%$ of participants matched the inclusion criteria, $10.0 \%$ were healthier (i.e. healthy BMI or no comorbidities) and $16.8 \%$ had higher weight related health risk than the targeted population (i.e. BMl of over $40 \mathrm{~kg} / \mathrm{m}^{2}$ or combination of obesity and comorbidities). For $24.3 \%$ of participants eligibility could not be checked, due to missing BMl-value or missing information about presence of comorbidities at baseline. The number of eligible participants did not differ between the programmes.

In interviews, HCPs reported that participants were mainly recruited by GP and practice nurse and some HCCs (also) recruited via physiotherapist or the dietician. In three HCCs the practice nurse or dietician actively searched through registries to recruit participants; in these HCCs 14, 20 and 21 participants were recruited. In terms of reach per HCP, a practice nurse mainly saw chronic patients, while other HCPs saw more people who had overweight or obesity without comorbidities ("I (practice nurse) mainly recruited patients with diabetes, while the GP and physiotherapist mainly recruited people who had obesity.'). If participants were recruited by GP, they often had wrong expectations; this was reported as a possible reason for drop-out by HCPs ('Sometimes the GP discussed it too briefly. Well, I think they weren't very motivated, so I often had to amend participants' expectations.').

According to questionnaires, $\mathbf{7 6 . 9 \%}$ of participants were referred by the GP to LSA for the BeweegKuur. In total, $\mathbf{8 0 . 9 \%}$ received approval by the GP to start in the BeweegKuur. The participants reported that their main reasons to participate mainly were to lose weight ( $\mathrm{n}$ $=242,58.9 \%)$, improve fitness $(n=196,47.7 \%)$, increase physical activity $(n=145,35.3 \%)$, improve health $(n=143,34.8 \%)$, decrease medication use $(n=98,23.8 \%)$ and the combination of both physical activity and diet $(n=87,21.2 \%)$. Only $6.6 \%(n=27)$ reported that improving their current unhealthy eating behaviour was a main reason to participate in the BeweegKuur. 
Table 5.I. Baseline characteristics of recruited participants

\begin{tabular}{|c|c|c|c|}
\hline & $\begin{array}{l}\text { Total } \\
(n=4 I I)\end{array}$ & $\begin{array}{l}\text { Start-up } \\
\text { programme } \\
(n=164)\end{array}$ & $\begin{array}{l}\text { Supervised } \\
\text { programme } \\
(n=247)\end{array}$ \\
\hline \multicolumn{4}{|l|}{$\operatorname{Sex}(\%)$} \\
\hline Male & $35.3(n=145)$ & $36.0(n=59)$ & $34.8(n=86)$ \\
\hline Female & $64.7(n=266)$ & $64.0(n=105)$ & $65.2(n=161)$ \\
\hline Age (mean years $\pm S D)$ & $55.1 \pm 12.4 \quad(n=411)$ & $53.8 \pm 12.4 \quad(n=164)$ & $55.9 \pm 12.3 \quad(n=247)$ \\
\hline \multicolumn{4}{|l|}{ Nationality (\%) } \\
\hline Dutch & $88.8(n=325)$ & $90.9(n=130)$ & $87.4(n=195)$ \\
\hline Other & $1 \mathrm{I} .2(\mathrm{n}=4 \mathrm{I})$ & $9.1(n=13)$ & $12.6(n=28)$ \\
\hline \multicolumn{4}{|l|}{ Educational level (\%) } \\
\hline Low & $40.3(n=146)$ & $37.3(n=53)$ & $42.3(n=93)$ \\
\hline Middle & $41.4(n=150)$ & 45. I $(n=64)$ & $39.1(n=86)$ \\
\hline High & $18.2(n=66)$ & $17.6(n=25)$ & $18.6(n=4 \mid)$ \\
\hline \multicolumn{4}{|l|}{ Occupation (\%) } \\
\hline Paid work & $41.0(n=150)$ & $41.3(n=59)$ & $40.8(n=91)$ \\
\hline Unpaid work & $22.7(n=83)$ & $27.3(n=39)$ & $19.7(n=44)$ \\
\hline Not working / studying & $36.3(n=133)$ & $31.5(n=45)$ & $39.5(n=88)$ \\
\hline \multicolumn{4}{|l|}{ Marital status (\%)* } \\
\hline Married & $65.6(n=240)$ & $61.5(n=88)$ & $68.2(n=152)$ \\
\hline Unmarried & $11.7(n=43)$ & $18.2(n=26)$ & $7.6(n=17)$ \\
\hline Cohabiting & $9.6(n=35)$ & $7.0(n=10)$ & $11.2(n=25)$ \\
\hline Divorced & $8.2(n=30)$ & $9.1(n=13)$ & $7.6(n=17)$ \\
\hline Widowed & $4.9(n=18)$ & $4.2(n=6)$ & $5.4(n=12)$ \\
\hline \multicolumn{4}{|l|}{ Body Mass Index } \\
\hline Mean \pm SD (n) & $34.5 \pm 4.4(n=368)$ & $35.0 \pm 4.6(n=145)$ & $34.2 \pm 4.2(n=223)$ \\
\hline$<30$ kg/m² (\%) & $16.6(n=61)$ & $14.5(n=24)$ & $17.9(n=40)$ \\
\hline $30-35$ kg/m² (\%) & $35.6(n=|3|)$ & $33.8(n=49)$ & $36.8(n=82)$ \\
\hline$\geq 35$ kg/m² (\%) & $47.8(n=176)$ & $51.7(n=75)$ & $45.3(n=101)$ \\
\hline
\end{tabular}

* Significant difference between start-up and supervised participants; $\mathrm{p}<0.05$ 


\section{Fidelity}

Except for one physiotherapist, all HCPs stated that they were trained in MI techniques and that they applied these techniques in meetings with the participants. HCPs graded their use of $\mathrm{Ml}$ techniques on average $6.9( \pm 0.8)$ on a 10 -point scale. There were no differences between type of HCP, HCCs and interventions.

In the interviews, all physiotherapists indicated that they made an exercise plan with the participants ('We tried to set up an individual exercise plan based on the Dutch norm for healthy physical activity and several functional tests.'), while $84.8 \%$ of the participants indicated that they set exercise goals or made an exercise plan with an HCP. The majority of the exercise plans or goals were made with physiotherapist (79.9\%). In total, $90.1 \%$ of start-up participants and $93.1 \%$ of supervised participants attended at least one meeting with physiotherapist, which would be a requirement to set exercise goals. Of six dieticians with whom the topic was discussed during the interviews, five made nutritional plans with the participants. One dietician did not plan individual meetings and therefore felt there was no opportunity to set individual goals. In the questionnaires, $73.9 \%$ of the participants mentioned that they made a nutritional plan or set nutritional goals with an HCP. The majority of the nutritional plans or goals were made with the dietician (91.7\%). Of start-up participants, $94.4 \%$ attended at least one individual dietician meeting essential for setting nutritional goals, in contrast to $63.5 \%$ in the supervised programme.

HCPs of five HCCs mentioned that the participants often required additional psychological counselling ('For a substantial number of participants, the BeweegKuur lacked guidance by a psychologist. When it becomes personal, several related emotional matters come up (...), but that was often difficult to expose, because we (as LSAs) have not been trained for that.'), and according to the HCPs this was due to the shift of target population from patients with type 2 diabetes to people who have overweight or obesity, prior to study start.

HCCs were aware of the study design, and all HCCs were allowed to offer the supervised programme prior to the study. Most HCPs from start-up HCCs felt their care had fallen short due to the fact they were not allowed to offer the supervised programme to the research population ('Although I did not express it to the participants, the fact that certain participants might benefit more from a more intensively guided programme did influence my thoughts.'). In interviews it appeared that HCPs expressed the belief that more exercise guidance was necessary to help this group to adopt a physically active lifestyle. Only one HCP reported that the start-up programme had been sufficient for the participants. The planning of individual meetings compared to group meetings and the reduced time investment were seen as advantages of the start-up programme by two HCPs. 


\section{Dose delivered}

Almost all (96.9\%) participants reported that their LSA had explained the intervention clearly at the start of the intervention. Median number of LSA meetings was higher in the start-up programme than in the supervised programme (table 5.2). There was a significant difference in number of LSA meetings between HCCs (range median number per HCC: 0 -6 ; Kruskal-Wallis, $p=0.008)$.

Table 5.2. Planned and actual dose delivered according to participant questionnaires.

\begin{tabular}{llllll}
\hline & \multicolumn{2}{l}{$\begin{array}{l}\text { Number of meetings } \\
\text { according to protocol }\end{array}$} & \multicolumn{2}{l}{$\begin{array}{l}\text { Attended number of meetings } \\
\text { (median }\end{array}$} \\
& Start-up & Supervised & Start-up & Supervised & P-value \\
\hline LSA meetings & 6 & 6 & $4(2-5)$ & $3(2-4)$ & 0.017 \\
Physiotherapist group meetings & - & $26-34$ & $0(0-9)$ & $16(3-24)$ & $<0.00 \mathrm{I}$ \\
Physiotherapist individual meetings & 6 & $6-7$ & $2(\mathrm{I}-5)$ & $0(0-2)$ & $<0.00 \mathrm{I}$ \\
Dietician group meetings & 7 & 7 & $2(0-5)$ & $3(0-4)$ & $\mathrm{NS}$ \\
Dietician individual meetings & 3 & 3 & $4(2-7)$ & $1(0-3)$ & $<0.00 \mathrm{I}$ \\
\hline NS = not significant & & & & &
\end{tabular}

One physiotherapist of the start-up programme planned group meetings with all HCPs following the intake meeting instead of the intended individual meetings with physiotherapist ('A one-time advice does not stick. We intensified this by assembling all involved HCPs, to maximise chance of success'). All HCPs stated that they individualised the programme due to either planning issues (holidays), health issues or made well-considered adjustments to individual participants' wishes and/or needs.

In comparison to the start-up programme, the total number of physiotherapist meetings was higher in the supervised programme ( 4 and 20 respectively; Mann-Whitney $U$ test, $P$ $<0.00 \mathrm{I}$ ), with on average more group meetings and fewer individual meetings (table 5.2). The individual physiotherapist meetings were not attended by $20.3 \%$ of participants in the start-up programme and by $53.8 \%$ of participants in the supervised programme. Within the start-up programme, the total number of physiotherapist meetings in the intervention period differed significantly between HCCs (range median number per HCC: 0 - I5; Kruskal-Wallis, $p=0.004$ ).

Half of the dieticians had typically offered individual meetings with participants. The other four dieticians planned individual meetings dependent on the participant ('For instance, I would say to participants, if you have quite a few questions or you would like some extra support, then I would advise one meeting per month.'). Main reasons for not planning individual 
meetings were lack of interest from participants or related costs ('Some participants did not want individual dietician meetings, because they had to pay for those meetings their selves.'). According to the interviews, the number of group meetings ranged from four to eight between dieticians. Two dieticians reported that participants perceived the scheduled seven group meetings to be too much, and therefore planned fewer meetings than prescribed by the protocol ('We planned fewer group meetings, just to assure adherence of participants.'). Four dieticians experienced difficulties in the group dynamics due to background differences between participants, specifically in terms of psychological issues, motivation, age, gender, intelligence and ethnic background ('A few participants said the level of the group meetings was too low to attend the meetings.'). According to the questionnaires, the number of individual meetings in the start-up programme was higher (table 5.2), but number of group meetings was equal in the two programmes. The number of participants that were referred to a dietician was significantly lower in the supervised group $(82.9 \%$ versus $67.5 \%$ ). There was a significant difference in number of dietician meetings between HCCs (range median number per HCC: 0 - 9.75; Kruskal-Wallis, $\mathrm{p}<0.00 \mathrm{I}$ ).

Of 226 participants who completed the questionnaire after 12 months, $40.7 \%$ reported the LSA had explicitly concluded the BeweegKuur intervention. The intervention was not concluded in $41.2 \%$ of the participants and $18.1 \%$ did not know.

\section{Dose received}

The participants' satisfaction with group meetings with physiotherapist and with the entire BeweegKuur guidance was higher in the supervised group than in the start-up group (table 5.3). The satisfaction with guidance by LSA and physiotherapist in groups differed between the HCCs (one-way ANOVA, $p=0.018$ and $p=0.021$ ).

Strategies to reduce drop-out consisted mainly of contacting a participant after no show via telephone or mail (reported by $72 \%$ of HCPs) and contacting other involved HCPs (28\% of HCPs). Two HCPs stated that they deviated from the protocol by adapting the planning of the meetings for individuals with high perceived drop-out risk and three HCPs explicitly discussed the reasons of no show with the participant to prevent future dropout. Two HCPs were unsure whether they should have put more effort in contacting participants to reduce drop-out, but they had been hindered by time constraints.

According to HCPs, reasons for non-adherence of participants were mainly physical problems or illness (reported by $68 \%$ of HCPs), lack of motivation (52\% of HCPs), unrealistic expectations towards intervention guidance ('Some people might not realise that the BeweegKuur requires own effort and activity.') or effects ('If it didn't quite work for a participant, they could become very critical about the intervention after three or four times.'; $48 \%$ 
of HCPs), practical issues such as holiday and work (48\% of HCPs), group meeting related issues ('Some persons did not feel comfortable in the group.'; $32 \%$ of HCPs) and (unexpected) costs of the guidance ( $20 \%$ of HCPs). Less mentioned reasons were low intelligence, private circumstances and that the project was too laborious. Based on the HCP records of each participant and personal communication between participants and researchers, $5 \mathrm{I}$ $(20.6 \%)$ in the supervised programme did not complete the planned full year and 38 (23.2\%) in the start-up programme (based on data of 10 start-up and 15 supervised HCCs and all registrations by researchers). Not all drop-outs of the BeweegKuur were registered, but the main reasons were health issues $(31.5 \%)$ and personal reasons $(10.1 \%)$.

The interviews revealed that the name of the BeweegKuur, literally translated 'Movement Therapy', could have led to wrong expectations of participants, possibly causing drop-outs ('Participants signed up for a movement therapy, so they did not expect nutritional guidance. I think that might have caused drop-outs in the initial phase of the intervention.').

Table 5.3. Dose received according to participant questionnaires.

\begin{tabular}{|c|c|c|c|}
\hline & \multicolumn{2}{|c|}{$\begin{array}{l}\text { Satisfaction (graded I-I0) } \\
(\text { mean } \pm s d)\end{array}$} & \multirow[b]{2}{*}{ P-value } \\
\hline & Start-up & Supervised & \\
\hline LSA meetings & $7.2 \pm 1.9$ & $7.5 \pm 1.7$ & NS \\
\hline Physiotherapist group meetings & $7.1 \pm 2.3$ & $8.0 \pm 1.3$ & 0.036 \\
\hline Physiotherapist individual meetings & $7.2 \pm 2.2$ & $7.7 \pm 1.5$ & NS \\
\hline Dietician group meetings & $7.2 \pm 2.0$ & $7.1 \pm 1.7$ & NS \\
\hline Dietician individual meetings & $7.3 \pm 1.9$ & $7.1 \pm 1.9$ & NS \\
\hline BeweegKuur overall & $7.1 \pm 1.8$ & $7.7 \pm 1.5$ & 0.044 \\
\hline
\end{tabular}

NS = not significant

\section{Context and implementation strategy}

The BeweegKuur was aimed to be covered by the basic health insurance scheme in 2012 (I52). However, this plan was abandoned after a change in government in 2010 (I53). While the initial development of the BeweegKuur was ordered by the ministry a few years earlier, the focus of the new minister of Health, Welfare and Sports was less on prevention.

HCPs reported in interviews that support of NISB was mainly experienced in the initial implementation phase of the programme, and was perceived to be less present at the time of the execution of study. However, information and material from NISB was continuously used for the guidance of participants. Main functional input from NISB was appreciated in 
the form of $\mathrm{MI}$ and BeweegKuur courses, log books for participants, protocols for guidance and participant presents (e.g. water bottles). Support to implement the BeweegKuur was mainly provided by the local ROS organisations. Satisfaction of HCPs with this support varied between HCCs. Some HCPs reported that they did not need support by ROS, because the intervention and collaborations were on track or because they did not believe the ROS could provide the help they needed. Other HCPs reported that the ROS did not have a great role in the BeweegKuur. Support of ROS seemed to reduce after it had become clear that the BeweegKuur would not be covered in the Dutch basic health insurance scheme. A few HCPs stated the support was completely terminated and this influenced continuation negatively ('All support ceased due to the governmental cutbacks. Then you realise how difficult it is to continue.').

The aim of the BeweegKuur was that after the one year intervention, participants would continue to exercise in one of the local facilities in the environment of the participant. However, identifying and mapping these facilities by the HCPs was problematic. Indecisiveness and uncertainty regarding whose responsibility it was and time constraints limited the process ('We had contact with ROS, because we both held the municipality responsible for the mapping of exercise facilities, but they refused to do that.'). In some HCCs, the municipality took responsibility to map the exercise facilities, and this was appreciated by the local HCPs. One HCP missed information and material for non-Dutch speaking eligible participants, hindering sufficient guidance for this group.

\section{Sustainability}

None of the HCCs intended to maintain the name BeweegKuur specifically after the completion of the study. Four HCCs (40\%) still offered a structured intervention to people who have overweight or related comorbidities, based on the BeweegKuur (e.g. cardiovascular risk management, type 2 diabetes, chronic obstructive pulmonary disorder). In most of these CLls, it depended on the participant whether guidance by a dietician was offered. Costs were covered by insurance of physiotherapy and/or dietary counselling, but part of the costs was also frequently paid by the participant. Three HCCs $(30 \%)$ intended to continue CLls in their practices and were still in the process of setting this up. Three HCCs (30\%) had no intention of continuing a structured CLI; however, in two of these HCCs, the interdisciplinary collaborations were utilised to refer people to the physiotherapist or the dietician for advice or guidance. HCCs (with the intention of) continuing a CLI, adapted the programme to their experiences, their daily practice and the individuals. 
HCPs stated that continuation of the BeweegKuur or a combined lifestyle programme for overweight people was hindered mainly by reimbursement issues ('Now that the project is not reimbursed by the government, I have no idea how to finance the BeweegKuur.'). A premise for sustainability of CLls was the availability of funding, such as an affordable participant contribution or reimbursement of programme aspects through the health insurance ('Reimbursement via the diagnosis-treatment combinations for diabetes still enables us to organise intervention aspects.'). Another facilitating factor was the collaboration with municipality in the form of local exercise coaches. Most HCPs reported that they were willing to look for funding; however, time constraints hindered them to do so. In addition, a few HCPs had applied for funding of a major health care funding institute; this was either unsuccessful or only postponed the termination of the programme.

In most HCCs (60\%), the discontinuation of funding led to termination of BeweegKuur implementation and execution. Five HCCs explicitly attributed the hindered continuation to changes of the political climate (i.e. less emphasis on prevention). One HCC attributed the discontinuation to organisational changes in their HCC and one HCC was dissatisfied with the $\mathrm{CLI}$ in their HCC. Also, the financial situation of participants was seen as hindering by HCPs, as not all participants had sufficient means to cover insurance costs for own account.

\section{Discussion}

The aim of the process evaluation was to provide insight into the implementation, execution and sustainability of a CLI in primary care. A newly composed framework was used to ensure structured and complete evaluation. Both HCPs and participants indicated that the participants' expectations of the intervention were often not met. Also, guidance was frequently not according to protocol and adherence differed between the two programmes and clusters. Nevertheless, in the intensive programme people received more physiotherapist supervision than in the start-up programme. Sustainability of the BeweegKuur was low; however, knowledge, experiences and networks from the implementation of the BeweegKuur were utilised in most HCCs to continue some form of combined lifestyle approach in primary health care.

Interestingly, a few HCPs stated that information provided by GPs prior to recruitment and the focus on physical activity in the intervention name sometimes led to wrong expectations. In addition, weight loss was the reason to participate in the majority of participants. This is in line with previous findings, showing that participants perceived the intervention to be successful when they lost weight (I22). Weight loss might be a false expectation, because the adoption of physical activity and a healthier diet does not 
necessarily lead to immediate weight loss $(154,155)$. Though the goal of the intervention is the adoption of a healthy lifestyle in terms of physical activity and dietary behaviour to improve health, not all participants realise this. Therefore, non-adherence in future studies might be reduced if expectations are more realistic and in line with the intervention.

The HCPs rated their application of $\mathrm{MI}$ on average a 6.9. An earlier study showed that $\mathrm{MI}$ was feasible in primary care and usable in diabetes care management (I56). In addition, MI has been shown to lead to significant weight loss (145). However, a study evaluating the quality of $\mathrm{MI}$ by means of observation, showed that practice nurses applied MI only partially (I57), indicating that HCPs may overestimate their skills in optimally applying $\mathrm{MI}$. Regardless, in our study participants were on average very satisfied with guidance by HCPs in the intervention. Participant questionnaires showed that number of physiotherapist meetings differed significantly between the start-up and supervised protocol, as anticipated. Although physiotherapist guidance should be the only guidance that differs between the two programmes, numbers of individual meetings with LSA and dietician were significantly lower in the supervised programme. Moreover, the proportion of participants which was referred to the dietician was approximately 15\% lower in the supervised programme. In addition, interviews revealed that the high amount of physiotherapist guidance and the sometimes unexpected nutritional aspects of the intervention might have reduced the number of attended dietary meetings. One could argue that the intensive guidance by physiotherapist makes guidance by LSA surplus and dietary change difficult. A study that also concerned a CLI found no effects on objectively measured health behaviours, and authors argued the disadvantage of targeting multiple lifestyle behaviours simultaneously (158). In a study evaluating implementation in a small amount of BeweegKuur HCCs, it had already been observed that guidance by a dietician was not performed according to protocol (159). Some dieticians indicated that the timing of meetings and their content were possible reasons for non-adherence (I59). The selective rejection of an intervention might have benefits, for instance in terms of feasibility or participant adherence, and is therefore not necessarily undesirable (160). However, a study by Rutten et al. showed that, during the BeweegKuur, motivation shift for dietary behaviour was small, possibly explained by the complexity of dietary behaviour. Participants in this study indicated that they were less satisfied with support by the LSA to improve dietary behaviour than physical activity (60). The findings of Rutten et al. (20I4) combined with the low number of attended meetings with the dietician might have caused the lack of motivation after four months of intervention. Even though the numbers of dietician and LSA meetings were lower in the supervised programme, our study showed that the participants in the supervised programme were more satisfied with the 
intervention than the participants in the start-up programme. Also, some HCPs believed the start-up programme did not offer sufficient guidance for all participants. This indicates that it would be preferable to tailor the guidance to individual needs and wishes. However, the difference in effectiveness between the two programme intensities and the possible influence of the number of meetings remain to be studied.

The type of participants that was reached seemed to differ between different HCPs who recruited participants. Since recruitment could be performed by all HCPs and this differed between HCCs, the participants' characteristics might have varied between HCCs, possibly affecting potential costs and outcomes. Nevertheless, relevant baseline characteristics of participants were not different between programmes. Cluster randomisation reduces risk of contamination and is particularly suitable to evaluate interventions implemented in various locations (I6I). A study describing the reach in a cluster randomised trial, showed that recruitment by HCPs who are not blinded, can lead to unequal distribution in the control and experimental group (162). In our study, motivation between HCCs might have differed, leading to the large variation in number of participants per HCC. This dissimilarity in motivation might have had consequences for programme execution during the study and underlines the importance of treating variation between HCCs as potential influence on cost-effectiveness.

Over the years, the BeweegKuur has been optimised based on advice from the ministry of Health, Welfare and Sports and on evaluations by Helmink et al. (57, I I I, I22, I63). In 2009, the Dutch government intended to include the BeweegKuur in the basic health insurance scheme (I52). Process data collected in 2010 showed that HCPs were motivated to implement and continue the BeweegKuur (I $2 I$ ). In addition, a study by Rutten et al. (2014) showed a shift to a more autonomous motivation for physical activity in BeweegKuur participants (60), which is assumed to precede the engagement in physical activity (120). However, after a change in government in 2010 , the intention of including BeweegKuur in the insurance scheme was abandoned $(153,163)$. According to the interviews in our study, this decision influenced implementation support by ROS, and because they had not anticipated on the lack of funding, the sustainability of this CLI was hindered (i.e. whether participation in a CLI was still possible at the HCC). Our finding that funding and external collaborations were perceived as key factors in sustainability of the CLI in the HCCs, is in line with Green \& Tones, who described the impact of lack of funding and collaborations (164). Although none of the HCCs has actually continued the BeweegKuur according to the protocol, most HCCs do offer lifestyle guidance in which strategies, experiences and collaborations from the BeweegKuur are employed. Adapting or selectively rejecting parts of an intervention is defined as re-invention, which might 
support the sustainability of an intervention in daily practice, because the users of the programme (i.e. the HCPs) adjust the programme to experiences, needs and possibilities of their own and of the participants (160). The recruitment of participants who strictly would not be eligible for the study, but were recruited anyway, based on HCPs' experiences, is also a form of re-invention. Nonetheless, most BeweegKuur intervention elements are essential for lifestyle change, such as goal setting and evaluation. It is therefore uncertain whether the interventions as they are currently offered will have similar costs and effects as the BeweegKuur we have been studying. Also, the low sustainability of the programme might be caused by the perceived lack of an implementation strategy (149). Although most HCPs were satisfied with the support by ROS and NISB during implementation, HCPs missed support in sourcing alternative sources of funding. After the decision not to include BeweegKuur in the basic insurance scheme, NISB focused on sustainable networks, and as anticipated, most HCCs still utilised networks formed during BeweegKuur. During future design and implementation of $\mathrm{CLI}$ in real world setting, care should be taken to plan not only implementation, but also sustainability of all aspects of the intervention required for the intended goal.

The HCPs in the current study might not be representative for the entire population in primary care, because they were participating in the BeweegKuur from an early stage, and could therefore be labelled as innovators and early adopters (121, 160). Accordingly, sustainability and the extent of programme adjustment might be higher due to the longer experience and familiarity with the programmes. Another limitation is the implementation of the supervised programme in control HCCs prior to this study, which has potentially influenced the degree of re-invention in the control HCCs. For instance, one of the interviewed physiotherapists from the start-up programme planned group instead of individual meetings, which might be triggered by the exposure to group meetings of the supervised programme prior to the study. However, this reflects the real world setting in which it is unavoidable that previous experiences potentially influence the degree of reinvention of other interventions. In addition, participant registration was used and interviews with HCPs were conducted after the study ended, possibly causing an increased risk of recall bias in both participants and HCPs. Nevertheless, by triangulating information from HCPs and participants in our evaluation, we attempted to minimise effects of recall bias.

The strength of our process evaluation is the application of a solid research framework to identify potential influences on costs and outcomes, but also to provide insights beneficial for future intervention implementation and studies. We have constructed and performed the process evaluation prior to the analyses and interpretation of (cost-)effectiveness, to 
ensure a full analysis of the factors with potential impact on the results. Also, the triangulation of participant and HCP data increased validity of our results.

\section{Conclusion}

Protocol adherence in our CLI was problematic in both HCPs and participants. Cluster randomisation was applied to decrease contamination, but also led to diversity in guidance. Guidance in all HCCs deviated from the protocol, and adherence differed between both programmes and clusters. Consequently, we showed that evaluation of (cost-)effectiveness should account for cluster differences, for instance by using multilevel analyses. The high amount of physical activity guidance seems to lead to a diminished opportunity for dietary change, so the guidance in CLls should be well-balanced to assist multiple behaviour change. An important lesson learned is that the liberty of re-inventing the CLI and political and financial facilitation seems to be crucial for the sustainability of the CLI, and should therefore be included in an implementation strategy in future interventions. 



\section{CHAPTER 6}

Effectiveness of 'BeweegKuur', a combined lifestyle intervention for overweight people in primary care

Brenda AJ Berendsen

Marike RC Hendriks

Geert M Rutten

Stef PJ Kremers

Hans HCM Savelberg

Nicolaas C Schaper 


\section{Abstract}

Background Combined lifestyle interventions (CLIs) have been shown to improve physical activity and dietary behaviour of overweight and obese adults. The dose of guidance within a CLI might influence effectiveness. This study assessed the effects of additional physical activity guidance in a CLI consisting of guidance by a lifestyle advisor (LSA), physical therapist and dietician in primary care setting.

Methods Thirty health care clusters (HCCs) were randomised into a control (start-up programme) or an experimental condition (supervised programme); $4 \mathrm{II}$ participants (BMI $34.5 \pm 4.4 \mathrm{~kg} / \mathrm{m}^{2}$ ) followed the programme allocated to their HCC. The start-up programme consisted of individual meetings with LSA, physiotherapist and dietician and group meetings with dietician, during a period of one year. The supervised programme included 26-34 additional physical activity group meetings guided by physiotherapist. The study included the one year intervention period and a two years follow-up. Primary outcome was self-reported physical activity at 12 and 24 months after baseline. Physical activity and diet were assessed every six months; clinical variables were assessed yearly. The additional effects of the supervised programme were studied with mixed models to account for clustering and repeated measures.

Results The primary outcome (physical activity) did not differ between the programmes. The only variable which changed differently between the programmes was waist circumference. In the supervised programme waist circumference decreased with an additional $4 \mathrm{~cm}$ at 12 months compared to the start-up programme. Several lifestyle and clinical variables improved equally in both programmes. Walking time increased at I2, 18 and 24 months compared to baseline with 88-104 minutes/week, sitting time decreased at six months with 43 minutes/day, BMI decreased at 12 and 24 months with 1.0 to I.I $\mathrm{kg} / \mathrm{m}^{2}$, waist circumference decreased at 12 and 24 months with $4.2-4.9 \mathrm{~cm}( \pm 0.7$ and \pm $0.9 \mathrm{~cm}$ ), and $\mathrm{HbAlc}$ decreased at 12 months with $1.6 \mathrm{mmol} / \mathrm{mol}$.

Conclusion Lifestyle and cardiovascular risk factors improved in both interventions; however, the addition of group meetings with physiotherapist aimed at physical activity did not lead to sustained enhanced benefits compared to the guidance in the start-up programme. Thus, the start-up programme (consisting of six individual meetings with LSA, ten meetings with dietician, and six individual meetings with physiotherapist) would be sufficient to facilitate a healthy lifestyle and improve health in a population with high weight related health risk. 


\section{Introduction}

A healthy lifestyle is an apparent opportunity to decrease health risks associated with overweight; however, the promotion of health enhancing behaviours remains challenging. In 2013 in the Netherlands, $44 \%$ of women and $53 \%$ of men were overweight (BMI > 25 $\mathrm{kg} / \mathrm{m}^{2}$ ), of which $13 \%$ and $\mathrm{I} I \%$ respectively were obese (BMI $\left.>30 \mathrm{~kg} / \mathrm{m}^{2}\right)(3)$. In addition, it has been estimated that $37 \%$ of the Dutch population does not meet the daily activity guidelines (3). By targeting both dietary behaviour and physical activity, the negative consequences of overweight can be prevented directly and indirectly, as evidence shows that an increase of physical activity can result in health benefits even in absence of weight loss (5).

Several combined lifestyle interventions are effective in reducing overweight and related risk for comorbidities $(30-33,36,51,165)$ and in one RCT a combined lifestyle intervention in subjects with impaired glucose tolerance was associated with a $58 \%$ risk reduction for diabetes, and a mean weight loss of $5 \%$ (5I). Specifically, the addition of physical activity counselling to a dietary intervention for obese participants has been shown to increase weight loss (3I). However, many efficacy trials have been performed in a controlled research setting, limiting practical generalisability. In the primary care setting, practice nurses have been suggested to function as lifestyle advisor (LSA) due to their expertise, contact with general practitioner (GP) and potential reach $(32,50)$. However, few intervention trials have been executed in the real-life primary care setting $(30,32)$, so the question remains whether combined lifestyle interventions are feasible and effective in daily practice.

Many lifestyle interventions suffer from high drop-out rates (I 19). To minimise drop-out, the BeweegKuur (Dutch for 'Exercise Therapy') has been developed and continuously adjusted in the Netherlands, as a lifestyle intervention in primary care addressing physical activity and diet with a focus on individualisation. Barriers towards adopting and sustaining a healthier lifestyle are decreased by means of Motivational Interviewing (MI), goal setting and experiencing physical activity (57). These strategies are applied by a team consisting of a GP, an LSA (often the practice nurse), a physiotherapist and a dietician (57). The BeweegKuur intervention shows similarities with the earlier studied Diabetes Prevention Program and the Look AHEAD trial, which have shown effects on weight and several clinical outcomes $(38,166)$. However, compared to the DPP and Look AHEAD study, goal setting within the current intervention was more tailored to the participant.

The BeweegKuur offers guidance in three doses; the so-called independent, the start-up and the supervised programme. The independent programme is intended for overweight individuals (BMI of 25 to $30 \mathrm{~kg} / \mathrm{m}^{2}$ ) without comorbidities, while the start-up programme 
and the supervised programme are intended for adults with a BMI of 25 to $35 \mathrm{~kg} / \mathrm{m}^{2}$ and suffer from comorbidities or a BMI of 35 to $40 \mathrm{~kg} / \mathrm{m}^{2}$ without comorbidities. The three programmes involve equal guidance by the LSA and dietician, but differ in terms of number of sessions with the physiotherapist (see table 6.I). The independent programme (one session with the physiotherapist) and start-up programme (six to seven sessions with the physiotherapist) have been argued to be effective in participants at risk of diabetes; however, the added value of the supervised programme (26 to 34 more sessions with the physiotherapist than offered in the start-up programme) in people with very high weight related health risk remained to be studied (63). More treatment sessions might enhance the effects on lifestyle (167); however a ceiling effect might occur when targeting multiple behaviours simultaneously (I58). Therefore, a clustered randomised controlled trial (cRCT) was designed to assess the difference in effects between the start-up and supervised programme in a group of participants with very high weight related health risk (a BMI between $25-35 \mathrm{~kg} / \mathrm{m}^{2}$ with comorbidities or a BMl between $35-40 \mathrm{~kg} / \mathrm{m}^{2}$ without comorbidities) (IOI) We hypothesised that the additional guidance of the supervised programme leads to an improved lifestyle (i.e. higher physical activity, healthier diet) and decreased health risk (i.e. lower BMI and clinical outcomes), compared to the start-up programme.

Table 6.I. Number of meetings in the independent (not part of this study), start-up (control condition) and supervised programme (experimental condition).

\begin{tabular}{llll}
\hline & $\begin{array}{l}\text { Independent } \\
\text { programme }\end{array}$ & $\begin{array}{l}\text { Start-up } \\
\text { programme }\end{array}$ & $\begin{array}{l}\text { Supervised } \\
\text { programme }\end{array}$ \\
\hline Lifestyle advisor meetings & 6 & 6 & 6 \\
Physiotherapist group meetings & - & - & $26-34$ \\
Physiotherapist individual meetings & I & 6 & $6-7$ \\
Dietician group meetings & 7 & 7 & 7 \\
Dietician individual meetings & 3 & 3 & 3 \\
\hline
\end{tabular}

\section{Methods}

Design

The design of the study has been described in detail elsewhere (I0I). The trial was developed as a cRCT with a control condition receiving an intervention that was planned to be usual care. However, the start-up programme of the BeweegKuur has not yet been reimbursed by the Dutch health care insurance, and therefore this study should now be referred to as a randomised comparative effectiveness trial, instead of an RCT. Cluster 
randomisation was applied to reduce the risk of contamination between participants, and the risk of bias at the level of the professionals involved. Thirty health care clusters (HCCs) were cluster randomised into the control condition (i.e. the start-up programme) or the experimental condition (i.e. the supervised programme). Each HCC consisted of a collaboration of GPs, LSAs, physiotherapists and dieticians. The interventions lasted one year, and participants were measured for two years after baseline to include a follow-up. Sample size was calculated to detect a difference of 50 minutes of MVPA between the programmes at twelve months after baseline. The study has been approved by the Medical Ethics Committee of the Maastricht University Medical Centre, has been registered with Current Controlled Trials (ISRCTN46574304) and was funded by The Netherlands Organization for Health Research and Development (ZonMW; project number: 123000002).

\section{Participants}

Inclusion criteria were (I) a BMI between $25-35 \mathrm{~kg} / \mathrm{m}^{2}$, combined with the following serious related comorbidities: sleep apnoea, arthritis, cardiovascular disease and/or type 2 diabetes; or (2) a BMI between $35-40 \mathrm{~kg} / \mathrm{m}^{2}$, but without these related serious comorbidities. In addition, participants should fail to meet the Dutch norm for healthy physical activity (30 minutes of moderate to vigorous physical activity (MVPA) on at least 5 days a week), and had to be sufficiently motivated to change their physical activity level and dietary behaviour (to be judged subjectively by the LSA). To assess motivation, the LSA had a first appraisal of participants' physical activity pattern and motivation by showing propositions to the participant and asking which most applied to their situation (e.g. 'I am currently not physically active and I do not intend to become physically active or "I am physically active, but not on a regular basis). The participant was asked to elucidate on the chosen proposition and the LSA used several questions to check whether the showed motivation was realistic (e.g. 'are you prepared to cycle two times a week?'). A detailed sample size calculation has been described earlier, resulting in a projected sample of 600 participants, accounting for the intra-cluster correlation, potential drop-out of participants and entire HCCs (IOI). Recruitment took place from July 2010 to October $20 \mathrm{II}$ and resulted in 4 II participants, of which 164 in the start-up (from I4 HCCs) and 247 in the supervised programme (from I 5 HCCs). Mean age was $55.1 \pm 12.4$ years, $35.3 \%$ was male and mean BMI was $34.5 \pm 4.4 \mathrm{~kg} / \mathrm{m}^{2}$ (I68). 


\section{Interventions}

Both programmes comprised guidance of one year; an elaborate description has been published earlier ( $\mathrm{IOI}$ ). The physiotherapist provided coaching with the aim to enable participants to exercise unsupervised in local exercise facilities. The amount and type of guidance by the physiotherapist differed between the programmes (table 6.I). The start-up programme included six individual meetings with the physiotherapist. The guidance by the physiotherapist in the supervised programme also included six or seven individual meetings and in addition 26-34 group meetings. The group meetings of the supervised programme took place in the first four months and consisted of physical exercise to overcome any barriers identified and increase physical capacity. The individual consultations with the physiotherapist in both programmes were aimed at identifying barriers to physical activity and drawing up a plan to remain physically active without the supervision by health care providers (HCPs).

The amount of guidance by the LSA and dietician was similar in the two BeweegKuur programmes; six individual meetings with the LSA, and three individual meetings and seven group meetings with the dietician. All HCPs in the team used $\mathrm{Ml}$ and goal setting to facilitate behaviour change and maintenance $(57,120)$. In both programmes, the LSA had a key role in supporting the participants and discussed progress and barriers of behavioural change. Sessions with the dietician consisted of nutritional recommendations, education, coping with high-risk situations, checking dietary behaviour and interaction between participants. Advice was based on various Dutch guidelines for diabetes, overweight and obesity $(124,125)$.

\section{Outcomes}

The primary outcome was self-reported level of physical activity instead of the physical activity estimations obtained with an accelerometer (CAM) (IOI). Unfortunately, the number of participants with valid measurements was low, and the questionable reliability of the CAM during MVPA (I02) limited the usability of the accelerometry data. Selfreported physical activity was measured every six months (at baseline, six months, twelve months, 18 months and 24 months) by respondents via the self-administered short version of the International Physical Activity Questionnaires (IPAQ), which is a reliable instrument for assessing daily physical activity (126, 169). Minutes of MVPA, walking and sitting, and total MET-minutes were calculated according to the IPAQ protocol (170). In our calculations, we arbitrary set the maximum time for walking and for moderate and vigorous activity as three hours and the minimum time for sitting time per day as 90 minutes. An additional self-administered question assessed whether participants adopted 
an independent physical exercise activity (i.e. exercise besides the guidance by the physiotherapist) after one year and after two years.

Dietary behaviour was retrieved with the short ENVET questionnaire (measured every six months) (I27, I7I). Specific dietary outcomes were weekly consumption of fruit, vegetables, snacks and candy.

The local HCP assessed the other secondary outcomes, i.e. body composition, clinical outcomes and functional capacity, at baseline, after one year and after two years. Body composition outcomes were height, weight, waist circumference and fat percentage. Fat percentage was assessed with a tetra polar bioelectrical impedance device (OMRON BF5II). Further, blood pressure was measured and blood samples were taken to assess the clinical outcomes $\mathrm{HbAlc}(\mathrm{mmol} / \mathrm{mol})$, total cholesterol $(\mathrm{mmol} / \mathrm{L}), \mathrm{HDL}(\mathrm{mmol} / \mathrm{L})$ and creatinine $(\mu \mathrm{mol} / \mathrm{L})$. Diabetic neuropathy was examined using a standardised Clininal Neurological Examination (Valk neuropathy test) (129), while hand grip strength (130, I3I) and the timed 'up and go' test were used as measures of functional capacity (I32). Aerobic capacity was estimated using the sub-maximal Åstrand cycle test by the physiotherapist (133, 134).

\section{Analyses}

Data were presented as mean \pm standard error, unless mentioned differently. Differences in baseline characteristics, rate of drop-out and adverse events between the two programmes were tested with t-tests or Chi-square tests. Descriptives and Chi-square tests were used to evaluate whether participants adopted an independent activity and whether participants complied with the Dutch physical activity norm of I50 minutes of MVPA per week.

Linear mixed model analysis techniques were applied to the longitudinally measured primary and secondary outcomes. The analyses involved a three level design with repeated measures as the first level (ARI covariance structure for serial correlation), participant as second level (unstructured covariance) and HCC as third level (unstructured covariance). Changes compared to baseline were assessed with pairwise comparisons, applying the Bonferroni correction (comparing four time points to baseline results with an alpha of $0.0125)$. Primary analyses were performed with MVPA time, walking time, sitting time and MET-minutes according to IPAQ as dependent variables. A priori chosen independent variables were moment of measurement and programme (start-up and supervised); a priori chosen covariates were age (I72), gender (I67), BMI at baseline $(172,173)$ and season $(174,175)$. Secondary analyses were performed with dietary behaviour, BMI, weight, waist circumference, fat percentage, $\mathrm{HbAlc}$, cholesterol, HDL and blood pressure 
as dependent variables. Linear mixed model analyses account for data missing at random, without imputation of missing data (176). However, to avoid inclusion of single data points, only participants with at least two measurements of the particular outcome were analysed. Intention-to-treat analyses were performed with primary outcomes, using multiple imputation of missing data to account for large loss to follow up. Exploratory analyses were done using the per protocol principle. In the first set of per protocol analyses, the participants were excluded who were registered as drop-out via HCP or via communication with researchers. In the second set of per protocol analyses, participants were also excluded when they had insufficient adherence to the allocated programme (i.e. no group meetings with physiotherapist and I -6 individual meetings with physiotherapist in the start-up programme or 20-40 group meetings with physiotherapist in the supervised programme). Analyses were done in SPSS 21.0 with a significance level of 0.05 , unless mentioned differently.

\section{Results}

\section{Baseline characteristics}

At baseline, demographics did not differ between the two study groups (table 6.2) (168). On average, participants in the supervised programme had higher values of MVPA time ( $P$ $=0.032)$ and total physical activity $(p=0.030)$. Main analyses were therefore corrected for these baseline differences.

\section{Drop-out and loss to follow-up}

Baseline clinical and functional measurements of $91.4 \%$ of start-up and $90.3 \%$ of the supervised participants were available (figure 6.1). Lack of data was most often caused by HCCs that did not provide the results or participants that dropped out immediately after recruitment. The baseline questionnaire was returned by $87.8 \%$ of participants in the startup and $89.1 \%$ in the supervised programme. At the second follow-up, two years after baseline, clinical and functional measurements were available of respectively $50.0 \%$ and 44. I\% of the participants in the start-up and supervised programme. Questionnaire return rates decreased to $51.2 \%$ and $52.6 \%$ at the two year follow-up measurement. 
Table 6.2. Baseline characteristics of participants in the start-up and supervised programme.

\begin{tabular}{|c|c|c|}
\hline & $\begin{array}{l}\text { Start-up programme } \\
(n=164)\end{array}$ & $\begin{array}{l}\text { Supervised programme } \\
(n=247)\end{array}$ \\
\hline Age (in years) & $54 \pm 12$ & $56 \pm 12$ \\
\hline Sex (\% Male) & 36 & 35 \\
\hline BMI (in kg/m²) & $35.0 \pm 4.5$ & $34.2 \pm 4.2$ \\
\hline Waist circumference $(\mathrm{cm})$ & $113.5 \pm 11.3$ & $1 \mid 2.8 \pm 11.1$ \\
\hline Diabetes (\% Yes) & 34 & 41 \\
\hline MVPA time (minutes/week)* & $249 \pm 317$ & $335 \pm 438$ \\
\hline Walking time (minutes/week) & $199 \pm 238$ & $229 \pm 307$ \\
\hline $\begin{array}{l}\text { Total physical activity } \\
\text { (METminutes/week)* }\end{array}$ & $1964 \pm 2040$ & $2591 \pm 3000$ \\
\hline Sitting time (minutes/day) & $385 \pm 188$ & $394 \pm 194$ \\
\hline $\begin{array}{l}\text { Compliance with physical activity } \\
\text { norm (\%) }\end{array}$ & 52 & 53 \\
\hline \multicolumn{3}{|l|}{ HbAl c (mmol/mol) } \\
\hline - Participants with diabetes & $54.5 \pm 15.3$ & $54.8 \pm 14.4$ \\
\hline - Participants without diabetes & $40.2 \pm 6.6$ & $38.3 \pm 5.6$ \\
\hline SBP $(\mathrm{mmHg})$ & $139 \pm 18$ & $136 \pm 16$ \\
\hline DBP (mmHg) & $85 \pm 11$ & $84 \pm 10$ \\
\hline
\end{tabular}

Data are percentage or mean \pm sd. BMI = Body Mass Index; MVPA = moderate to vigorous physical activity; $\mathrm{SBP}=$ systolic blood pressure; $\mathrm{DBP}=$ diastolic blood pressure.

*Significant difference between the programmes $(p<0.05)$.

Of 4 II participants, a total of 89 participants (22\%) did not complete the 12 month intervention period. Chi-square tests showed that percentage of drop-outs did not differ between the two programmes $(p=0.643$ ) or for other baseline characteristics (e.g. sex and eligibility). Sixteen participants (five in control and II in experimental group) dropped out immediately after recruitment, the reasons were: the HCC was unable to start up the study $(n=3)$, recruitment mistakes $(n=2)$, health issues $(n=I)$ and unknown $(n=10)$. Within the 12 months of guidance, 73 participants dropped out, of which 33 in the control and 40 in the supervised programme. Reasons for dropping out were health issues (i.e. adverse events, $n=27)$, unknown $(n=17)$, private circumstances $(n=9)$, lack of time $(n=$ $6)$, not motivated $(n=5)$, moved $(n=4)$, financial issues $(n=2)$, wrong expectations $(n=$ $2)$ and fear of physical activity $(n=1)$. 
Within the 24 months of follow-up, 86 adverse events were registered (42 in start-up and 44 in supervised programme, e.g. orthopaedic surgery), of which 26 were classified as serious ( 18 in start-up and 8 in supervised programme; e.g. diagnosis of cancer or cardiovascular disease). Five adverse events were attributable to participation in the intervention and/or trial, of which four were skin irritation due to wearing the activity monitor (activity monitor data was not used in this article, see discussion) and one participant had fallen during supervised exercise without serious consequences. Two participants were excluded from analyses due to pregnancy. The percentage of (serious) adverse events was not different between the programmes.

\section{Physical activity}

No differences were observed between the programmes in any of the primary outcome parameters of self-reported physical activity. In both programmes, mixed models showed changes over time for walking and sitting time, but no changes in weekly minutes of MVPA and total physical activity (MET-minutes) (table 6.3). Walking time increased compared to baseline at 12, 18 and 24 months with respectively $88 \pm 28,106 \pm 31$ and $104 \pm 29$ minutes per week in the entire group $(p=0.008 ; p=0.002$ and $p=0.001$, respectively). After six months, daily sitting time had decreased with $43 \pm 14$ minutes $(p=0.008)$. Overall, at 12 and 24 months, $54.7 \%$ and $52.5 \%$ of participants complied to the Dutch physical activity norm (no differences from baseline). At 12 and 24 months, $77.1 \%$ and 93.9\% of participants reported to have adopted an independent physical exercise activity. The percentage of participants who complied to the Dutch physical activity norm and who adopted an independent activity did not differ between programmes. Repeating the analyses after imputation of missing data did not change the results. 


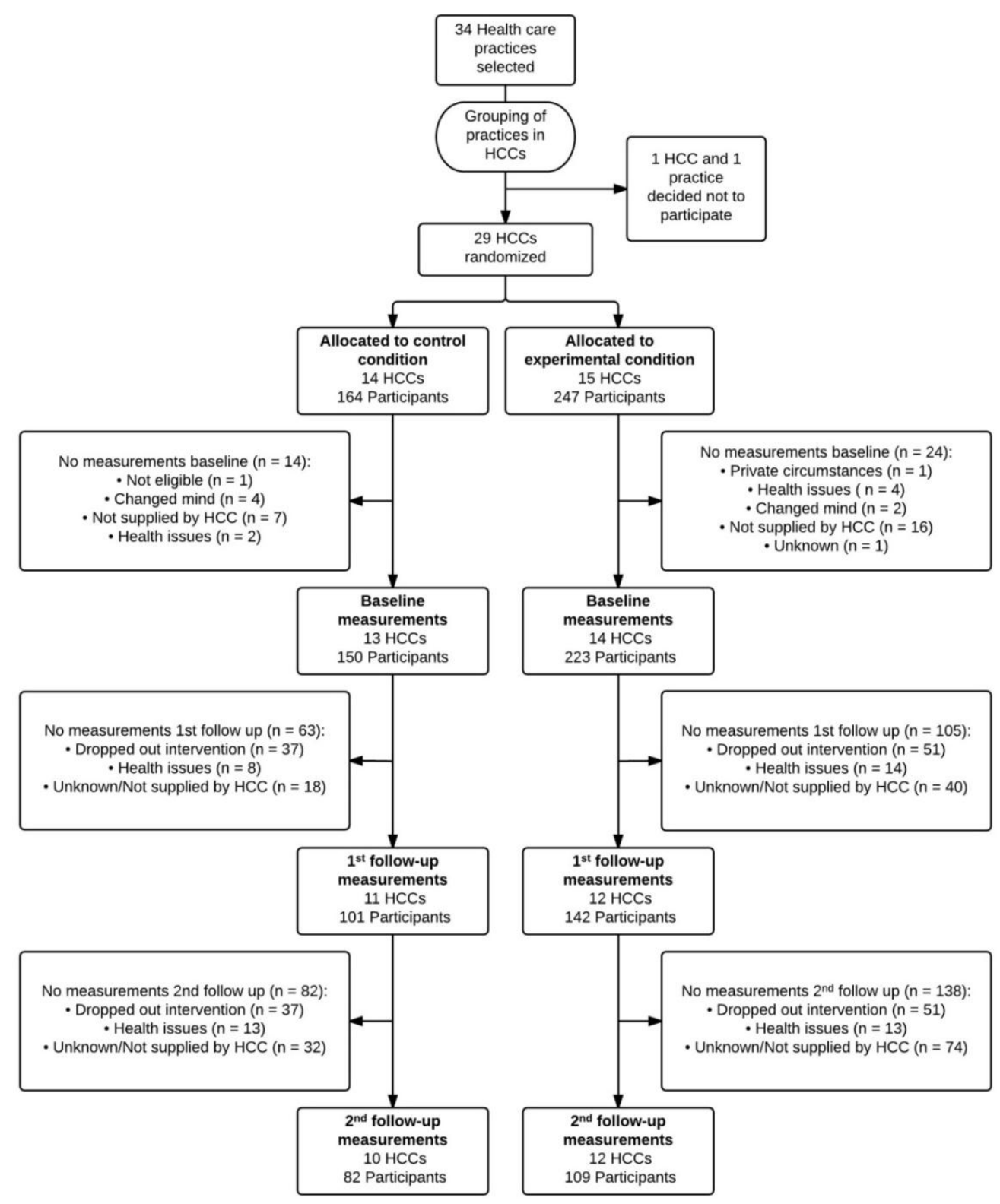

Figure 6.I. Flow diagram of recruitment of health care clusters (HCC) and participants, drop-out and annual measurements. 
Diet

Dietary habits showed changes over time in the entire group (table 6.3). Consumption of fruit increased at all moments compared to baseline, ranging from an average increase of $\mathrm{I} . \mathrm{I} \pm 0.4$ to $\mathrm{I} .6 \pm 0.4$ pieces per week ( $\mathrm{p}$-values ranging from $0.00 \mathrm{I}$ to 0.036 ). Weekly vegetable consumption increased at 12 and 18 months compared to baseline with respectively $3.4 \pm 0.9$ and $3.0 \pm 0.9$ table spoons $(p<0.001$ and $p=0.002)$. Intake of fatty snacks decreased compared to baseline at 12 , 18 and 24 months, with $0.4 \pm 0.1$ to $0.5 \pm$ 0.1 times per week ( $p$-values ranging from 0.001 to 0.022 ). Weekly intake of candy decreased at six, 12 and 18 months with $1.0 \pm 0.2$ (all $P<0.001$ ). There were no differences in dietary behaviour change between the BeweegKuur programmes.

Health related outcomes

In the entire group, BMI decreased with respectively $I .1 \pm 0.2 \mathrm{~kg} / \mathrm{m}^{2}$ at 12 months and 1.0 $\pm 0.2 \mathrm{~kg} / \mathrm{m}^{2}$ at 24 months compared to baseline (figure 6.2a and table 6.4; $\mathrm{p}<0.00 \mathrm{I}$ ), with no differences between the programmes. Overall, waist circumference decreased with respectively $4.9 \pm 0.7 \mathrm{~cm}$ and $4.2 \pm 0.9 \mathrm{~cm}$ at 12 and 24 months compared to baseline (figure 6.2b; $\mathrm{P}<0.00 \mathrm{I}$ ). The change in waist circumference over time was different between the two programmes $(p=0.0 \mathrm{II})$; the decrease in waist circumference at 12 months was $4.0 \mathrm{~cm}$ larger in the supervised programme, compared to the start-up programme.

There were no differences between programmes in terms of blood values and blood pressure. HbAlc values decreased after one year with $1.6 \pm 0.6 \mathrm{mmol} / \mathrm{mol}(p=0.019)$, with an interaction effect of presence of diabetes $(\mathrm{p}<0.00 \mathrm{I})$. Creatinine, cholesterol and HDL levels did not change over time. Diastolic blood pressure did not change, but systolic blood pressure was on average $4.3 \pm 1.3 \mathrm{mmHg}$ lower at 12 months compared to baseline $(p=0.001)$.

The timed 'up and go' test was performed $0.26 \pm 0.07$ seconds faster at 12 months compared to baseline $(P<0.001)$. Changes were not different between the two programmes. Hand grip strength, level of neuropathy and $\mathrm{VO}_{2}$-values from Åstrand submaximal test did not change over time. 
Table 6.3. Differences in lifestyle (i.e. physical activity, sitting time and diet) over time and between programmes.

\begin{tabular}{|c|c|c|c|c|c|c|c|c|}
\hline & $\mathbf{N}$ & Programme & 6 months & 12 months & 18 months & 24 months & $\begin{array}{l}\text { p-value } \\
\text { programme }\end{array}$ & $\begin{array}{l}\text { p-value } \\
\text { time }\end{array}$ \\
\hline $\begin{array}{l}\text { MVPA time } \\
\text { (minutes/week) }\end{array}$ & 242 & $\begin{array}{l}\text { Start-up } \\
\text { Supervised }\end{array}$ & $\begin{array}{l}16 \pm 52 \\
-10 \pm 41\end{array}$ & $\begin{array}{l}10 \mid \pm 44 \\
-10 \pm 42\end{array}$ & $\begin{array}{l}115 \pm 47 \\
14 \pm 47\end{array}$ & $\begin{array}{l}160 \pm 52 \\
-7 \pm 41\end{array}$ & 0.724 & 0.236 \\
\hline $\begin{array}{l}\text { Walking time } \\
\text { (minutes/week) }\end{array}$ & 240 & $\begin{array}{l}\text { Start-up } \\
\text { Supervised }\end{array}$ & $\begin{array}{l}64 \pm 31 \\
51 \pm 54\end{array}$ & $\begin{array}{l}112 \pm 36 * \\
69 \pm 32 *\end{array}$ & $\begin{array}{l}109 \pm 34^{*} \\
118 \pm 40^{*}\end{array}$ & $\begin{array}{l}101 \pm 38^{*} \\
83 \pm 35^{*}\end{array}$ & 0.792 & 0.000 \\
\hline $\begin{array}{l}\text { Total physical activity } \\
\text { (METminutes/week) }\end{array}$ & 206 & $\begin{array}{l}\text { Start-up } \\
\text { Supervised }\end{array}$ & $\begin{array}{l}208 \pm 340 \\
68 \pm 298\end{array}$ & $\begin{array}{l}829 \pm 278 \\
284 \pm 293\end{array}$ & $\begin{array}{l}901 \pm 329 \\
676 \pm 305\end{array}$ & $\begin{array}{l}911 \pm 316 \\
311 \pm 323\end{array}$ & 0.901 & 0.182 \\
\hline $\begin{array}{l}\text { Sitting time } \\
\text { (minutes/day) }\end{array}$ & 248 & $\begin{array}{l}\text { Start-up } \\
\text { Supervised }\end{array}$ & $\begin{array}{l}-62 \pm 18^{*} \\
-40 \pm 18^{*}\end{array}$ & $\begin{array}{l}-7 \pm 24 \\
-16 \pm 18\end{array}$ & $\begin{array}{l}-36 \pm 22 \\
-35 \pm 20\end{array}$ & $\begin{array}{l}-12 \pm 23 \\
-11 \pm 19\end{array}$ & 0.209 & 0.019 \\
\hline $\begin{array}{l}\text { Fruit consumption } \\
\text { (pieces/week) }\end{array}$ & 256 & $\begin{array}{l}\text { Start-up } \\
\text { Supervised }\end{array}$ & $\begin{array}{l}0.7 \pm 0.7^{*} \\
2.0 \pm 0.4^{*}\end{array}$ & $\begin{array}{l}0.4 \pm 0.7^{*} \\
1.8 \pm 0.5^{*}\end{array}$ & $\begin{array}{l}\mathrm{I} .5 \pm 0.7^{*} \\
\mathrm{I} .4 \pm 0.5^{*}\end{array}$ & $\begin{array}{l}0.8 \pm 0.7^{*} \\
1.6 \pm 0.6^{*}\end{array}$ & 0.349 & 0.001 \\
\hline $\begin{array}{l}\text { Vegetable consumption } \\
\text { (table spoons/week) }\end{array}$ & 253 & $\begin{array}{l}\text { Start-up } \\
\text { Supervised }\end{array}$ & $\begin{array}{l}2.6 \pm 1.2 \\
0.8 \pm 1.1\end{array}$ & $\mathrm{I} . \mathrm{I} \pm \mathrm{I} .3^{*}$ & $3.6 \pm 1.5^{*}$ & $\begin{array}{l}1.0 \pm 1.4 \\
0.8 \pm 1.0\end{array}$ & 0.927 & 0.000 \\
\hline $\begin{array}{l}\text { Snack consumption } \\
\text { (pieces/week) }\end{array}$ & 255 & $\begin{array}{l}\text { Start-up } \\
\text { Supervised }\end{array}$ & $\begin{array}{l}-0.5 \pm 0.2 \\
-0.2 \pm 0.2\end{array}$ & $\begin{array}{l}-0.3 \pm 0.2 * \\
-0.5 \pm 0.2 *\end{array}$ & $\begin{array}{l}-0.6 \pm 0.2^{*} \\
-0.5 \pm 0.2^{*}\end{array}$ & $\begin{array}{l}-0.3 \pm 0.2^{*} \\
-0.6 \pm 0.2^{*}\end{array}$ & 0.891 & 0.001 \\
\hline $\begin{array}{l}\text { Candy consumption } \\
\text { (pieces/week) }\end{array}$ & 256 & $\begin{array}{l}\text { Start-up } \\
\text { Supervised }\end{array}$ & $\begin{array}{l}-1.3 \pm 0.4^{*} \\
-0.7 \pm 0.3^{*}\end{array}$ & $\begin{array}{l}-1.0 \pm 0.5^{*} \\
-1.1 \pm 0.4^{*}\end{array}$ & $\begin{array}{l}-0.7 \pm 0.4^{*} \\
-1.2 \pm 0.4^{*}\end{array}$ & $\begin{array}{l}-0.2 \pm 0.5 \\
-0.7 \pm 0.3\end{array}$ & 0.141 & 0.000 \\
\hline
\end{tabular}

Data are mean \pm SE.

MVPA = moderate to vigorous physical activity.

*Significantly different from baseline. 
Table 6.4. Differences in health parameters over time and between programmes.

\begin{tabular}{|c|c|c|c|c|c|c|}
\hline & $\mathbf{N}$ & Programme & 12 months & 24 months & p-value programme & p-value time \\
\hline BMI $\left(\mathrm{kg} / \mathrm{m}^{2}\right)$ & 218 & $\begin{array}{l}\text { Start-up } \\
\text { Supervised }\end{array}$ & $\begin{array}{l}-0.8 \pm 0.3^{*} \\
-1.2 \pm 0.2^{*}\end{array}$ & $\begin{array}{l}-0.7 \pm 0.3^{*} \\
-1.5 \pm 0.3^{*}\end{array}$ & 0.934 & 0.000 \\
\hline Weight (kg) & 229 & $\begin{array}{l}\text { Start-up } \\
\text { Supervised }\end{array}$ & $\begin{array}{l}-2.5 \pm 0.7^{*} \\
-3.7 \pm 0.7^{*}\end{array}$ & $\begin{array}{l}-1.2 \pm 0.8^{*} \\
-3.8 \pm 0.8^{*}\end{array}$ & 0.603 & 0.000 \\
\hline Waist circumference $(\mathrm{cm})$ & 192 & $\begin{array}{l}\text { Start-up } \\
\text { Supervised }\end{array}$ & $\begin{array}{l}-2.9 \pm 1.0^{*} \\
-6.9 \pm 0.9 *\end{array}$ & $\begin{array}{l}-3.1 \pm 1.5^{*} \\
-5.3 \pm 0.9 *\end{array}$ & 0.011 & 0.000 \\
\hline Fat percentage (\%) & 209 & $\begin{array}{l}\text { Start-up } \\
\text { Supervised }\end{array}$ & $\begin{array}{l}-1.3 \pm 0.4^{*} \\
-1.9 \pm 0.4^{*}\end{array}$ & $\begin{array}{l}-1.3 \pm 0.8^{*} \\
-2.7 \pm 0.7^{*}\end{array}$ & 0.671 & 0.000 \\
\hline HbAlc (mmol/mol) & 142 & $\begin{array}{l}\text { Start-up } \\
\text { Supervised }\end{array}$ & $\begin{array}{l}-1.8 \pm 0.8^{*} \\
-2.5 \pm 1.1^{*}\end{array}$ & $\begin{array}{l}0.2 \pm 1.0 \\
0.0 \pm 1.3\end{array}$ & 0.573 & 0.010 \\
\hline Total cholesterol ( $\mathrm{mmol} / \mathrm{L})$ & 169 & $\begin{array}{l}\text { Start-up } \\
\text { Supervised }\end{array}$ & $\begin{array}{l}-0.06 \pm 0.11 \\
-0.29 \pm 0.10\end{array}$ & $\begin{array}{l}-0.06 \pm 0.12 \\
-0.19 \pm 0.15\end{array}$ & 0.635 & 0.163 \\
\hline HDL (mmol/L) & 162 & $\begin{array}{l}\text { Start-up } \\
\text { Supervised }\end{array}$ & $\begin{array}{l}-0.12 \pm 0.04 \\
-0.10 \pm 0.10\end{array}$ & $\begin{array}{l}-0.05 \pm 0.04 \\
-0.13 \pm 0.18\end{array}$ & 0.155 & 0.336 \\
\hline SBP (mmHg) & 213 & $\begin{array}{l}\text { Start-up } \\
\text { Supervised }\end{array}$ & $\begin{array}{l}-6.2 \pm 1.8^{*} \\
-1.2 \pm 1.4^{*}\end{array}$ & $\begin{array}{l}-1.8 \pm 1.7 \\
0.4 \pm 1.8\end{array}$ & 0.069 & 0.002 \\
\hline DBP (mmHg) & 214 & $\begin{array}{l}\text { Start-up } \\
\text { Supervised }\end{array}$ & $\begin{array}{l}-2.9 \pm 1.3 \\
0.5 \pm 1.0\end{array}$ & $\begin{array}{l}-2.5 \pm 1.2 \\
-0.7 \pm 1.1\end{array}$ & 0.147 & 0.062 \\
\hline
\end{tabular}

Data are mean \pm SE.

$\mathrm{BMI}=$ Body Mass Index; $\mathrm{SBP}=$ systolic blood pressure; $\mathrm{DBP}=$ diastolic blood pressure.

*Significantly different from baseline. 

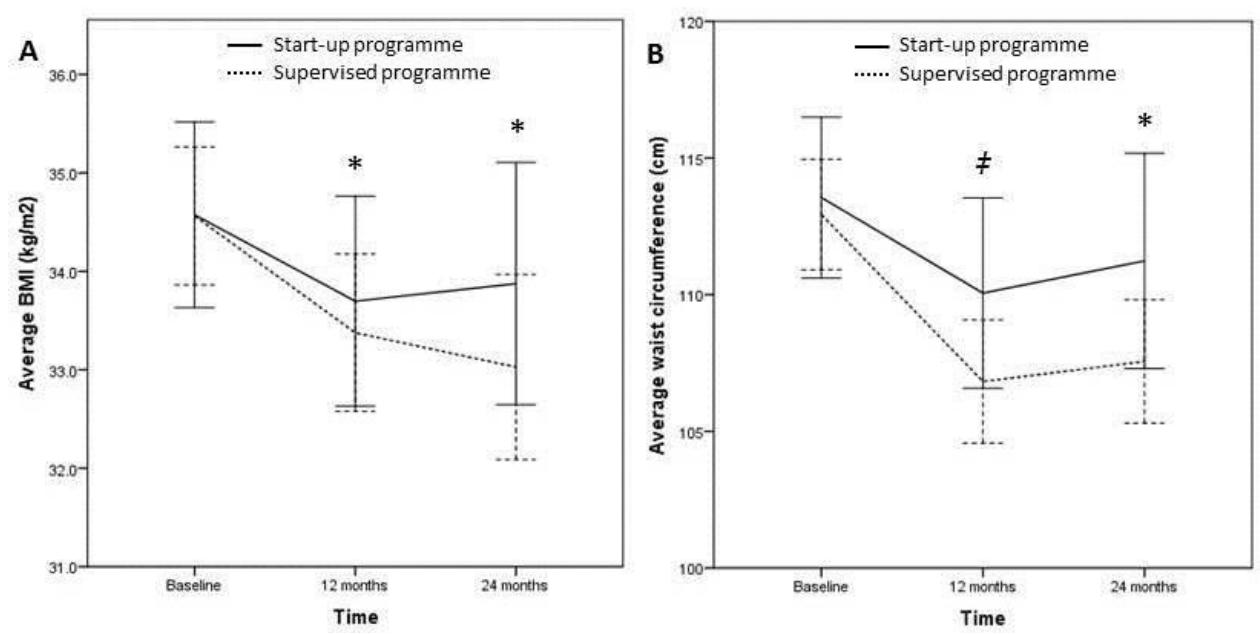

Figure 6.2a-b. Changes in BMl (A) and waist circumference (B) with $95 \%$ confidence intervals.

‡Significantly different from baseline and between the control and experimental programme; *Significantly different from baseline regardless of programme

Per protocol analyses

In the exploratory per protocol analyses there were no significant differences between the programmes, except for hand grip strength. Overall hand grip strength decreased with 4.3 $\pm 1.5 \mathrm{kgf}$ at 24 months compared to baseline $(p=0.012)$; but in the start-up programme strength decreased with $7.4 \pm 4$. I kgf and in the supervised group increased with $1.5 \pm 0.9$ kgf. With regards to changes over time, the difference in walking time between 24 months and baseline was not significant and sitting time was not significantly decreased at six months in the first set of per protocol analyses (excluding dropped out participants). Diastolic blood pressure decreased significantly at 24 months, compared to baseline $(p=$ 0.048). Other findings were similar to the primary analyses. The second set of per protocol analyses were not executed (excluding dropped out participants and participants with insufficient adherence), because there were too few participants meeting the selection criteria.

\section{Discussion}

This study showed that a moderate and high dose combined lifestyle intervention both had beneficial effects on lifestyle behaviours as well as on cardiovascular risk profile. However, there were no added benefits of the additional physical exercise training on the primary outcome of physical activity or on the secondary outcomes of dietary intake and most cardiovascular risk factors. Waist circumference at 12 months was the only 
secondary outcome with differences between groups. Participants in the supervised programme had a significantly larger decrease of waist circumference at 12 months; however, this additional effect was not sustained at 24 months. We conclude that the higher amount of physical activity under guidance of the physiotherapist in the supervised programme did not lead to additional effects compared to the start-up programme.

Extra group meetings guided by the physiotherapist in the supervised programme were expected to lead to enhanced effects on lifestyle and cardiovascular risk profile, compared to the start-up programme (IOI). These physiotherapist group meetings were held during the first months of the programme and very likely contributed to the larger decrease in waist circumference at 12 months. In the second year no such meetings were held and the difference in waist circumference between the two programmes disappeared. Although our data indicate that participants adopted independent physical exercises and maintained an overall decrease in waist circumference, perhaps the additional effects of the supervised programme would be sustained if the supervised group exercise meetings were continued. The supervised exercises under guidance of the physiotherapist and the interaction between participants in a group were hypothesised to lower barriers towards adopting independent exercise. However, first of all, we did not observe a difference between the two programmes in the percentage of participants that adopted independent exercise, indicating that the group exercise meetings did not result in lower barriers towards physical activity, i.e. a ceiling effect of physical activity guidance occurred. Secondly, the feasibility of executing the supervised programme according to protocol was lower compared to the start-up programme, as reported elsewhere (168). According to protocol the supervised programme should have consisted of both individual consultations with a physiotherapist and group meetings under guidance of the physiotherapist. Strikingly, most participants in the supervised programme did not attend any individual meetings with the physiotherapist and also attended fewer individual meetings with the LSA and dietician than the participants in the start-up programme (168). Therefore, it is questionable whether these participants received the intended coaching via Motivational Interviewing and goal setting. Experience built up during supervised exercise sessions can increase feelings of competence, which according to Self Determination Theory are important for the development of autonomous motivation and it can also decrease barriers towards exercise, but goal setting is essential for behaviour change maintenance (58). Potentially, effects in the supervised programme would have been larger if participants had attended more individual meetings. It has recently been proposed that processes involved at improving diet and increasing physical activity might interfere with each other in interventions targeting these behaviours simultaneously (I77). In addition, 
our process evaluation showed that, in the supervised programme, the number of attended group meetings was lower than the protocol prescribed (168). Hence, although current findings indicate that targeting physical activity and diet simultaneously can be effective in primary care setting, the addition of two or three group meetings per week was not realistic. In short, a ceiling effect might have occurred due to feasibility issues.

After the intervention period of one year and the follow-up at two years, weight had decreased with $3.4 \%$ and $2.7 \%$ respectively compared to baseline in participants in both programmes, revealing a sustained weight loss after termination of guidance by HCPs. Especially the decrease in waist circumference, as discussed above, is of importance, given its beneficial effects on mortality and the development of diabetes mellitus (178, 179). These results are in agreement with an earlier study arguing that the BeweegKuur programme would be a suitable intervention for people with (pre-)diabetes (63). In addition, observational studies indicated beneficial effects on lifestyle and motivation of the independent and start-up programme after implementation of the BeweegKuur programmes in real world setting $(60,180)$. We found a significant decrease in BMI, waist circumference and fat percentage. Comparable lifestyle interventions have also shown positive results with regard to body composition $(30-33,36,51,115)$. The decreases in $\mathrm{BMI}$ and waist circumference in the current study were markedly larger than found in three studies which examined an intervention consisting of counselling sessions aimed at physical activity and diet compared to a usual care group $(30,32,36)$. These studies either consisted of fewer sessions (32) or the population had lower BMI at baseline $(30,36)$, decreasing the potential for effects. In comparison, other intervention studies reported larger effects on weight loss $(3 \mathrm{I}, 33,5 \mathrm{I}, 1 \mathrm{I5})$. Most of these studies comprised a population with higher BMI $(3 \mathrm{I}, 5 \mathrm{I}, \mathrm{II})$ or diabetes (33) and were executed in a controlled experimental setting ( $3 \mathrm{I}, 5 \mathrm{I}, \mathrm{I} I 5)$, which might have increased the opportunity for effects. The Look AHEAD study showed that weight regain after the initial intervention period was diminished due to long-term monthly guidance $(33,167)$, suggesting that sustained guidance might be necessary to sustain effects. The BeweegKuur guidance lasted one year, so the maintenance of weight loss and waist circumference during follow-up was accomplished without intervention. Therefore, especially in this realworld setting, the fact that the effects of the BeweegKuur were sustained during follow-up is encouraging.

The BeweegKuur has been hypothesised to suffer from less drop-out and non-adherence by utilising Motivational Interviewing and goal setting (57). The percentage of participants that did not drop out can be considered to be high. Unfortunately, participants' adherence in primary care based interventions is not always reported (30), but a review showed that 
adherence to lifestyle programmes in type 2 diabetes generally varies between 10 and $80 \%$ (I19). HCPs stated in the process evaluation that they attempted to reduce nonadherence and drop-out by adjusting the programme (i.e. re-invention) to individuals' wishes and needs (168). Another primary care based study showed no evident doseresponse relationship between the attendance of counselling sessions and clinical outcomes (32). However, the authors argued that the deviations from the protocol were part of the nature of the study (32), similar to our current study. Potentially, the real-life setting and the programme adjustments by HCPs have actually led to a decreased dropout in the BeweegKuur intervention.

Another interesting finding from the current study was that walking time increased, but sitting time did not increase. Results from previous studies indicated that compensation of increased physical activity in terms of energy expenditure might occur when a lifestyle intervention is aimed at MVPA, by decreasing light physical activity (30) or by increasing sedentary time (180). This compensation is undesirable, as it has been argued that physical activity and sedentary time have an independent influence on health $(I I-I 3,27)$. A recent study reported that this compensatory mechanism can be counteracted by including strategies that aim to increase non-exercise physical activity and reduce sedentary time $(\mid 8 I)$. Within the current study the increase in walking time was not associated with a compensatory increase in sitting time, but in future interventions the beneficial effects might be enhanced by targeting not only on physical exercise, but also on reducing sedentary time and increasing non-exercise physical activity.

The prospective design of the current study is one of its strengths; also, the inclusion of objectively measured health parameters, such as BMI, fat percentage and other cardiovascular risk factors strengthen our conclusions. The study was performed in primary care practices with local health care providers; the pragmatic design and setting maximises generalisability of our findings to daily practice. This probably also led to a more flexible execution of the intervention, with however a higher loss to follow-up compared to lab-based trials $(5 \mathrm{I}, \mathrm{II})$. An additional limitation was that we measured lifestyle changes with questionnaires, while better objective measures are available, especially for quantifying physical activity and sitting (102). The current study originally included measurements with the CAM tri-axial activity monitor, which has been shown to be valid in classifying postures $(81,102)$. However, collected data was not used since the reliability of the physical activity assessment at moderate and high doses was questionable (102) and utilising activity monitoring devices in such a large scale in a real-world intervention study proved to be difficult, because initialisation, application and data extraction had to be done by local HCPs. The physical activity data and sitting time were 
derived from the IPAQ short form and might not be an accurate reflection of actual behaviour due to social desirability and uncertainty regarding validity (182). Because participants could feel obliged to their LSA to have increased their physical activity level, we provided and collected the questionnaires via mail to minimise social desirability of the primary outcomes. And although this instrument is able to measure improvements in behaviour (126, 169), potential differences between the two programmes might have remained undetected due to lack of sensitivity of the IPAQ. In addition, actual recruitment did not meet the calculated sample size, so there might be a lack of power. Post hoc calculations with the acquired sample size and self-reported MVPA showed that a difference of 172 minutes of MVPA per week could be detected. Planned exercise within the supervised programme should account for 120 to 180 minutes of extra MVPA per week compared to the start-up programme. In addition, daily physical activity was promoted during the group meetings with the physiotherapist. Nevertheless, the a priori sample size calculation assumed a difference of 50 minutes of MVPA measured by accelerometers. Therefore, differences caused by the additional guidance in the supervised programme might have remained undetected in the current trial.

Because the goal of this trial was to determine the additional effects of a higher dose intervention compared to a medium dose, we did not include a control group receiving no intervention, which limits the conclusions about the effects of the two BeweegKuur programmes. Nevertheless, previous studies showed comparable results regarding effectiveness of the BeweegKuur $(60,63,180)$ and similar interventions $(30-33,36,5 \mathrm{I}, \mathrm{II}$, 165). In addition, the beneficial changes in health risk after two years of participants in the BeweegKuur programmes should be viewed in the perspective that these parameters are in this population expected to deteriorate over time $(183,184)$.

\section{Conclusion}

In conclusion, participants in both BeweegKuur programmes showed sustained beneficial changes in physical activity behaviour and diet compared to baseline accompanied with decreased BMI, waist circumference and $\mathrm{HbAlc}$. No differences were found between the supervised programme and the control condition, i.e. the start-up programme. Providing a large number of group meetings to facilitate physical activity did not lead to more success in terms of lifestyle and health compared to a limited number of individual physical activity sessions. Thus, the start-up programme (consisting of six individual meetings with the LSA, ten meetings with dietician, and six individual meetings with physiotherapist) would be sufficient to facilitate a healthy lifestyle and improve health in a population with high weight related health risk. 

CHAPTER 7

General discussion 
Overweight is one of the most important risk factors for type 2 diabetes and cardiovascular disease, mediated by factors like insulin resistance, high blood pressure and dyslipidaemia (I). Furthermore, lifestyle behaviours related to excess weight account for a significant burden of disease, with an estimated 12.5 million deaths attributable to physical inactivity and diet globally (I). Different effective strategies are possible to facilitate weight loss and prevent related non-communicable diseases, such as medical treatment, bariatric surgery and lifestyle coaching (14). Unfortunately, medical treatment is often accompanied by adverse effects and complications might occur due to medication interactions (16). Bariatric surgery is also associated with adverse effects (I5), and insurance coverage is conditional (185), limiting accessibility. Combined lifestyle intervention offers a valuable method for prevention and treatment of overweight, by targeting the excess weight itself, but also physical activity and diet.

Lifestyle interventions are designed to improve one or more behaviours related to overweight and obesity, in particular physical activity and diet. Physical activity and diet can be illustrated as two sides of a scale, and people have to find a balance between the energy intake (i.e. diet) and energy expenditure (i.e. physical activity). Weight ga in will occur in case of a long-term higher intake than expenditure (2). In addition to excess weight, physical inactivity has a negative effect on health $(186,187)$. Therefore, lifestyle interventions aimed at physical activity and diet can improve health both directly and indirectly. In addition, lifestyle interventions have been shown to improve other aspects, like fitness (188), cognition (189-191), urinary continence (39), mobility (40), quality of life $(42,192)$ and health care use (4I). However, lifestyle intervention programmes have been criticised for the low sustainability of lifestyle changes, the limited number of costeffectiveness studies and the relative small or even absent effects on cardiovascular end points $(43,193)$. A considerable amount of research has already addressed the effects of lifestyle interventions and knowledge is increasing rapidly. But the impact and optimal dose and content of lifestyle counselling remain to be determined, especially when implemented in actual primary care.

This thesis investigated the additional effect of group meetings with a physiotherapist in a combined lifestyle intervention, the BeweegKuur. The BeweegKuur was aimed at enhancing physical activity and improving dietary behaviour, by providing multidisciplinary guidance in primary care for one year. To assess effects realistically for future recommendations, the trial was set in actual primary care and was performed by local health care providers (HCPs). In addition, methods for measuring physical activity were also studied in this thesis. 
This chapter relates the findings from the research in this thesis with existing literature, addressing I) the effectiveness of the BeweegKuur and similar interventions, 2) the impact of a higher dose of guidance aimed at physical activity on lifestyle and health related outcomes, 3 ) the implementation and continuation of the BeweegKuur in primary care and 4) the measurement of physical activity. Further, methodological considerations describe the strengths and limitations of the studies presented in this thesis. Finally, this chapter concludes with the implications for research and clinical practice, finalising a general conclusion.

\section{EFFECTIVENESS OF COMBINED LIFESTYLE INTERVENTIONS}

As discussed above, a combined lifestyle intervention can lead to favourable changes in weight, quality of life, fitness and metabolic risk (33, 34, 42, 188, 192). As concluded in chapter 6, self-reported walking time, self-reported sitting time, self-reported dietary habits, weight, body composition, $\mathrm{HbAlc}$ and systolic blood pressure improved compared to baseline in participants in both BeweegKuur programmes. This is in line with an earlier study that indicated that participants' motivation towards physical activity improved during the first four months of the BeweegKuur (60). These are promising findings, yet the primary outcome, moderate to vigorous physical activity (MVPA, i.e. activities with MET-values of 3.0 to 6.0, not including walking), did not change over time (CHAPTER 6). Physical activity interventions have often focused on MVPA as an outcome, because physical activity guidelines promoted 30 minutes of MVPA per day in adults, disregarding light intensity physical activity (i.e. activities with MET-values of under 3.0) and sedentary time (i.e. sitting or reclining with MET-values of under 1.5). However, recent research focussing on physical activity behaviour revealed a potential, important role of physical activity at light intensity in cardio-metabolic health $(13,27)$. Furthermore, sedentary time has been associated with the metabolic syndrome (e.g. insulin resistance and dyslipidaemia) (I2, I3, 194). Light intensity physical activity and sedentary time offer great opportunities for health improvement, because change with respect to these behaviours are more realistic to achieve than an increase of MVPA (184). In response, several countries have recently included the advice to minimise prolonged sedentary time in their physical activity guidelines (195, 196), but in the Netherlands new guidelines implementing this new knowledge are yet to be developed. Regardless of the lack of change in MVPA in the current BeweegKuur trial, participants did report a sustained increase in walking time of 88 minutes per week after 12 months and 104 minutes per week after 24 months compared to baseline (CHAPTER 6). The change in walking time and the change in BMI were 
comparable to observations in an earlier BeweegKuur study, using the same self-report instrument $(180,197)$. It is difficult to compare the changes in weight and walking time, as well as their interrelationship, between studies, because almost always other variables changed, such as MVPA or diet. In addition, our study compared the effects of two programmes differing in dose of guidance and was not designed to assess differences compared to a control group receiving no intervention. Nonetheless, our findings seem to fit in the existing evidence, as other studies observed that an increase of walking time with $17 \%$ was accompanied with $0.7 \mathrm{~kg}$ weight loss after six months (32) or that a $20 \%$ increase of walking time was accompanied with $10-12 \mathrm{~kg}$ weight loss after twelve months (3I). Regular walking is best categorised as a light or moderate intensity physical activity (a walking speed of $3.4 \mathrm{~km}$ per hour is associated with 2.0 METs and $6.4 \mathrm{~km}$ per hour is associated with 5.0 METs). Furthermore, an increase in physical activity by walking can either be undertaken as exercise (i.e. for health or pleasure) or as a non-exercise activity (e.g. walking at home) (198). Each hour of brisk walking relates to a decreased risk for obesity of $24 \%$; standing or walking at home has been associated with a decreased risk for obesity of $9 \%$ (199). In addition, an increase of walking has been associated with improved lipid homeostasis and insulin sensitivity (7), lowered risk for type 2 diabetes $(199,200)$, improved bone and joint health (20I) and decreased frailty (202). An increase in walking time might therefore be very important for achieving long-term health benefits. The decreases in systolic blood pressure and $\mathrm{HbAlc}$ values of participants in the BeweegKuur reinforce this notion (CHAPTER 6).

The increase in self-reported walking time compared to baseline (as described in chapter 6) in combination with a reduced or unaltered self-reported sitting time, indicates that people did not seem to compensate for their increased physical activity by adopting other health threatening behaviour. The adoption of exercise training can lead to a decrease in non-exercise activity, especially in older adults, thereby negating the effect of the training on daily energy expenditure $(203,204)$. Also, results from earlier studies indicated that compensation in sedentary time or light intensity physical activity might occur when lifestyle interventions focus on moderate intensity physical activity $(30,180)$. But, a recent review revealed that combined lifestyle interventions (targeting diet and physical activity or sedentary behaviour) are capable of reducing sedentary time, with moderate to high quality evidence (205). This review is in line with our current finding that while walking time increased, sitting time decreased with $43 \pm 14$ minutes per day at six months (CHAPTER 6). Nevertheless, after six months, sitting time returned to baseline values, so also targeting sedentary time in the physical therapist meetings in the BeweegKuur should 
be advised to prevent relapse and achieve long-term health benefits of reduced sitting time, in addition to the health benefits of increased physical activity.

Also very promising were the improved dietary habits over time in the BeweegKuur participants (CHAPTER 6), because the estimated number of worldwide deaths attributable to a diet low in fruit approaches 5 million (I). Fruit consumption increased with $1.6 \pm 0.4$ pieces per week and vegetable consumption increased with $3.4 \pm 0.9$ table spoons per week. A recent meta-analyses showed that the promotion of fruit and vegetable consumption does not cause weight loss (206). Nonetheless, both fruit and vegetable consumption have been associated with (cardiovascular) mortality $(2 \mathrm{I})$ and an additional serving of fruit or vegetables per day (i.e. seven per week) is related to a $5-6 \%$ decrease of all-cause mortality (2I). Although the increase in fruit and vegetable consumption in our research sample was not of this magnitude, the achieved change might still be beneficial for health, as mortality mainly decreases non-linearly with a threshold of five servings per day (2I). As participants at baseline had an average weekly consumption of $10.1 \pm 0.5$ pieces of fruit and $25.3 \pm 0.9$ table spoons of 50 grams of vegetables, converted to a total of nearly 4 fruit or vegetables servings per day (according to the 77 grams table spoons in the review by Wang et al. (2I)), the weekly addition of 1.6 pieces of fruit and 3.4 table spoons of vegetables may be clinically relevant. Furthermore, snack consumption decreased (CHAPTER 6), which has also been related to cardio-metabolic health (20). A systematic review revealed a lack of evidence regarding the effects of nutritional counselling in primary care (207), but our current findings indicate that the guidance in the BeweegKuur intervention may improve dietary habits. The combination of enhanced walking time and improved diet may have an accumulating effect on health, as dietary aspects and physical (in)activity were argued to have the largest disease burden in terms of disability-adjusted life years (DALYs) (I).

An average weight loss of $3.5 \pm 0.6 \mathrm{~kg}$ after twelve months of behavioural counselling is in line with the findings of a recent systematic review concerning behavioural treatment of obesity in primary care (48). Also, the observed decrease in waist circumference, is in line with other studies $(3 \mathrm{I}-33,36,5 \mathrm{I})$. Especially the maintained improvements of weight loss and waist circumference after follow-up (two years from baseline) are remarkable, as other research has uncovered the challenges of sustaining weight loss without continued guidance (208). Waist circumference has been related to an increased risk for type 2 diabetes and to mortality $(209,210)$, so a lifestyle intervention of one year which might decrease these risks in the long term is very valuable. 


\section{IS MORE GUIDANCE MORE EFFECTIVE?}

The BeweegKuur could be provided with a different amount of guidance by the physiotherapist in three doses, the independent exercise programme, the start-up exercise programme and the supervised exercise programme. Participants are included in one of the three programmes based on the combination of BMI and the presence of comorbidities. The availability of different dosages of guidance by the physiotherapist is grounded on the premise that persons with comorbidities or more severe obesity perceive more barriers towards a new lifestyle and therefore require more sessions comprising Motivational Interviewing and physical activity (57). Needless to say, the programmes differ in their costs.

The programme with the highest dose of guidance by the physiotherapist, the supervised exercise programme, is intended for participants with a BMl between 25 and $35 \mathrm{~kg} / \mathrm{m}^{2}$ with comorbidities or a BMI between 35 and $40 \mathrm{~kg} / \mathrm{m}^{2}$ without comorbidities. The question in the current BeweegKuur trial (CHAPTERS 4 and 6) was whether the supervised exercise programme yielded extra effects in this specific group, compared to the start-up exercise programme which was considered to be (cost-)effective in the general population with pre-diabetes (63).

The addition of group meetings and supervised exercise were hypothesised to decrease the drop-out rate, and thereby increase the effects of the intervention, because group cohesion can stimulate participants to adhere to the programme $(61,62)$. However, we revealed that participating in the supervised programme did not decrease drop-out risk (CHAPTER 6). Our process evaluation revealed that the number of group meetings attended in the supervised programme was lower than prescribed in the protocol (CHAPTER 5), potentially weakening the effects of group cohesion. Perhaps more important was that participants in the supervised programme attended less individual meetings with the LSA, the physiotherapist and the dietician than participants in the startup programme (CHAPTER 4). These individual meetings were specifically aimed at setting personal and realistic goals, by means of Motivational Interviewing (I20). Therefore, participants in the supervised programme might have been less stimulated to set personal and realistic goals compared to the participants in the start-up programme.

Evidence regarding the optimal amount and type of guidance and the potential ceiling effect of the guidance in primary care is inconsistent. The question regarding guidance dose can be viewed in two ways, I) what are the immediate effects during the most intensive intervention period and 2) what is the maintenance of the intervention effects after the intervention is completed? The current BeweegKuur trial addressed both issues. 
The two programmes we compared had different numbers of sessions with the physiotherapist in the first three months of intervention. After this initial high dose period, guidance was equal in both programmes (CHAPTER 4). Results indicated that the extra sessions did not enhance improvements of lifestyle, weight and health, as physical activity, dietary habits, weight and blood values were not different between the two programmes (CHAPTER 6). In agreement, an RCT in outpatient clinic setting (i.e. primary care) showed that a relatively simple and low dose intervention using goal setting and Motivational Interviewing led to beneficial changes in PA and weight (2II). In contrast, an RCT by Hardcastle et al. revealed significant associations between number of counselling sessions and several health outcomes, e.g. body weight, blood pressure and cholesterol, in participants very similar to our study participants (patients at risk of coronary heart disease of which $20 \%$ and $79 \%$ respectively were overweight or obese) (32). Although lifestyle behaviours were not related to number of sessions, the authors stated that this could be explained by the self-reported measures or by insufficient power (32). In addition, a systematic review of weight loss interventions in primary care revealed that higher weight loss was achieved in interventions with more sessions (12-26 sessions compared to less than 12 sessions) (2I2). Here, an important note to make is that the review did not take into account whether the contents of the sessions used behavioural change techniques, such as Motivational Interviewing. The application of behavioural change techniques, such as goal setting and Motivational Interviewing, could be essential for lifestyle changes. To summarize, some counselling would be better than only screening and identifying risk factors (2|3), and adding extra sessions of counselling is probably not effective without using the techniques based on behavioural change theories.

Next to the lack of short term differences between the programmes, our two year followup data suggests that the extra sessions in the BeweegKuur in the first three months did not affect maintenance of the improvements (CHAPTER 6). Data from the Look AHEAD trial showed that the amount of guidance in the second till fourth year was related to weight loss maintenance in overweight or obese participants with type 2 diabetes, concluding that more guidance in a later intervention phase might be beneficial in maintaining healthy lifestyle and weight loss (167). The supervised programme of the BeweegKuur consisted of more guidance by the physiotherapist, but these sessions were all planned in the first three months of the intervention. While most lifestyle changes were sustained after two years, the addition of follow-up sessions at a later stage in the BeweegKuur (i.e. after the initial intervention phase) could have prevented that some of the dietary habits, HbAlc and blood pressure increased to baseline values at the follow-up of two years. 
Targeting multiple behaviours in one intervention has been shown to be more effective than targeting only diet or PA in overweight and obese adults $(48,214,215)$. However, this is not consistently true (I58). Possibly practical issues during the execution of multiple intervention aspects in collaboration with other professionals in daily primary care are limiting factors. The BeweegKuur process evaluation revealed some problematic aspects related to the fact that multiple behaviours were targeted within the same time window. Although it seems that targeting both diet and physical activity simultaneously might substantially increase awareness about health enhancing behaviour, it might not be feasible to attend all sessions planned in the supervised condition (26 to 34 group sessions with physiotherapist, in combination with the BeweegKuur standard six LSA sessions, six or seven individual sessions with physiotherapist and ten sessions with dietician). The sessions with the physiotherapist were all planned during the first three months of intervention, in addition to the first meetings with LSA and the first meeting with the dietician. So especially in the first intervention phase, people can be overwhelmed by the amount of guidance and sessions. And although participants were stimulated to set their own feasible goals, disappointment of not reaching one goal (e.g. PA goal) might influence the self-efficacy of reaching a different goal (e.g. diet goal), by causing a decreased confidence in personal competence (58). Other studies also noted the challenges in targeting physical activity and dietary habits in one intervention $(158,177,216)$. The cognitive effort required for changing dietary habits might interfere with adopting a physically active lifestyle (177). Nevertheless, many programmes intervening physical activity and diet simultaneously have been shown to positively affect diet, physical activity, weight and health (3I-36). Moreover, although the extra sessions with the physiotherapist in the BeweegKuur supervised programme did not have beneficial effects compared to the start-up programme, they did not negatively influence dietary changes. So with regards to lifestyle, weight and health, the addition of extra sessions might be optimal when they are distributed over a longer period, e.g. several years.

\section{COMBINED LIFESTYLE INTERVENTIONS IN PRIMARY CARE}

Primary care has been advocated to be a good setting for the treatment of obesity (48). However, real-life trials, including the current BeweegKuur trial, reveal challenges (CHAPTER 6). Since the first prototype of the BeweegKuur in 2007, the programmes have been monitored, improved and studied extensively (I63). Especially Helmink et al. have done a great deal of work in describing, evaluating and adapting the BeweegKuur programmes $(57,121,122,163,179,180,197,217,218)$, promising good results of the 
BeweegKuur in people with (pre-)diabetes. In addition, a review argued that the BeweegKuur was an effective method to stimulate healthy behaviour, especially in the population with high weight related health risk (63). Seemingly in contrast to this, no differences were detected in a study in which exercise level and health status were compared between people who did $(n=186)$ and did not participate $(n=2632)$ in the BeweegKuur programmes in 2008 (54). In 2008, the BeweegKuur was implemented as a prototype, of which Helmink et al. wrote a monitoring report (217) and performed a study of motivational factors in HCPs (I2I). Since then, the BeweegKuur has been altered, e.g. the amount of dietary guidance was enhanced and mapping of local exercise facilities was improved (217), into the programmes as described by Rutten et al. (60) and ourselves (CHAPTER 4). Nevertheless, Linmans et al. (54) might have touched an important issue in their discussion regarding the feasibility of lifestyle interventions in the primary care setting. Our process evaluation showed that execution of the programmes was not according to protocol, and the number of attended meetings was lower than prescribed (CHAPTER 5). Another process evaluation of the BeweegKuur programmes showed similar results (159). For a large part, the non-adherence to the programme was due to planning by HCPs, implying a certain (time and/or financial) investment threshold limiting the amount of feasible guidance in Dutch primary care. Correspondingly, the HCPs stated that funding played a role in the discontinuity of the interventions in the locations previously offering the BeweegKuur (CHAPTER 5). Undoubtedly, a facilitating context (e.g. financial certainty, being able to invest time and opportunities for attending refresher courses) is essential for the accurate execution and sustained availability of interventions in primary care.

As described above, facilitation is required for the execution and continuation of lifestyle interventions in clinical practice in the long term; however, flexibility within the intervention is also a key factor for HCPs to continue the intervention in the primary care. Adjustments of an innovation during implementation are therefore important; this process is described with the term 're-invention' (160). Some re-invention should be possible to ensure feasibility in daily practice, and to sustain the motivation of HCPs. As stated before, a number of HCPs in our study deliberately adapted the number of meetings with participants, for mainly practical reasons and own views. Although the perception of the HCP might not be entirely correct or stimulating behaviour change according to the selfdetermination theory (58), it is important that HCPs have the liberty to alter the intervention to suit their style, working schedule, existing collaborations and other responsibilities. However, intervention effects can only be guaranteed under the condition that the main mechanisms that bring about behavioural change are preserved. Particularly 
the participants in the supervised programme did not receive the prescribed number of individual meetings with any of the involved HCPs, while these meetings were specifically directed at maintaining behavioural change based on the self-determination theory (i.e. Motivational Interviewing and goal setting) (58). Strikingly, 53.8\% of the supervised group attended no individual meetings with the physiotherapist aimed at the identification of barriers and goals specifically for physical activity (CHAPTER 5). Thus, these participants might not have profited optimally from the behavioural change techniques included in the BeweegKuur. Future lifestyle studies should clearly define aspects that can be re-invented and which cannot, to ensure quality and effectiveness of the intervention.

The ultimate goal of preventive research is the implementation of (cost-)effective methods in usual care, in order to enhance health, control medical expenditure and thereby sustain the Dutch health care system (219). For long-term continuation of an intervention, optimal embedment in current practice is essential. Primary care practices participating in research can most likely be labelled as innovators and early adopters, i.e. persons or organisations embracing innovations before they are adopted on great scale (160). Nationwide adoption of an intervention might enhance long-term continuity in daily practice. It has been suggested that including the BeweegKuur programmes in the basic Dutch health insurance might stimulate continuity, because financial reimbursement can persuade the so-called early majority, late majority and laggards to adopt the intervention (I2I). The fifteen practices included in earlier research were highly motivated to continue the intervention in the long run (I2I). However, these promising results appeared to be undermined by financial issues. In 2010, the Dutch government decided not to include the BeweegKuur in the basic health insurance due to high costs in the short-term (I53). In addition, the current ministry of Health, Welfare and Sports holds the opinion that people are responsible for their own health and, therefore, the ministry does not stimulate reimbursement of health enhancing lifestyle interventions (I53). Unfortunately, prioritizing lifestyle interventions in decision making for health care reimbursements is challenging, because quantification of the costs and effects of these interventions often require longterm studies for which funding sources are scarce in contrast to pharmaceutical research (219). Therefore, the current BeweegKuur trial aimed to assess long-term costs and effects (CHAPTER 4). Nevertheless, the lack of future funding by the government was a major barrier for primary care practices to continue the intervention. Our process evaluation showed that $30 \%$ of participating primary practices stated to completely cease the implementation of combined lifestyle interventions (CHAPTER 5). Apparently, the lack of funding plays a crucial role in continuation of innovations, and sufficient time and energy should be invested in the development of long-term and reliable funding. Prior to and 
during the current trial, the BeweegKuur was freely accessible for eligible participants, i.e. the intervention could be described as an unconditional financial incentive. Research suggests that offering guidance or services as financial incentive might be beneficial for physical activity behaviour (220). An obstacle in reimbursement of prevention programmes is the decision-making framework that is currently employed in the Netherlands. Decision criteria are often not applicable to both treatment and prevention, often leading to low priority and budgets for the latter (219). However, the Dutch National Health Care Institute has advised to reimburse combined lifestyle interventions for overweight, because these interventions were deemed to be necessary and (cost-)effective (64). Nonetheless, the Dutch government decided not to include combined lifestyle interventions in the basic health insurance, hindering the sustainable embedment in the health care system.

\section{MEASUREMENT OF PHYSICAL ACTIVITY}

Most large-scale studies aimed at behavioural change rely on subjective measurement methods, due to feasibility issues and costs of an objective alternative. Objective measurement of quality of diet in real life is virtually impossible as only observation and analyses of the actual consumed food would be a true value of intake. Recently, objective measurement of physical activity by accelerometry has been gaining interest and robustness. Accelerometers are wearable devices which measure accelerations in one to three directions and thereby estimate frequency and intensity of movement (66). In addition, accelerometers can fulfil the increasing need for objective estimates of sitting time, due to the probable influence of sedentary behaviour (i.e. sitting and lying) on cardio-metabolic health $(12,13)$. Our study evaluating three accelerometers revealed that only the thigh-worn devices provided valid posture classification, probably because the orientation of the upper leg is very distinctive during sedentary postures and standing (CHAPTER 2). Even though posture classification of the CAM was valid, the study indicated that the measurements by the CAM might be compromised by questionable reliability during physical activity at moderate to high intensity and by mediocre user friendliness (CHAPTER 2), prohibiting us from using the data registered by the CAMs in the BeweegKuur study. Nonetheless, tri-axial accelerometers were proven to be able to provide valid and reliable estimates of time spent sitting, standing and walking (CHAPTER 2). In addition, accelerometers can promote physical activity in daily life when applied in a feedback system (22I). Therefore, it seems to be advisable to apply accelerometers in real-life intervention studies aimed at increasing physical activity. But, some problems exist 
in applying accelerometers in this setting. First, repeating measurements in a large-scale study proved to be very difficult, due to both problems regarding wear by participants (i.e. refusing to wear for motivational reasons, as observed in the user friendliness results in chapter 2) and practical issues (planning visits and hardware errors). Second, the outcomes differ between the devices, limiting comparability with other studies or lifestyle interventions. There is no consensus as to which outcome would be most appropriate in terms of validity and reliability. Mostly used outcomes are activity counts and time spent in activity. Both outcomes have their own set of considerations limiting comparability between devices: activity counts are calculated in a black box by manufacturer's software, whereas the estimate of time spent in activity depends on the activity threshold chosen by manufacturer or researcher. Third, issues during measurement, such as non-wear (CHAPTER 3), are often not handled, possibly leading to wrong estimates and thereby leading to conclusions based on incorrect data. Moreover, previously validated datahandling (e.g. non-wear algorithm and epoch length) only applies to the studied population and might not yield valid estimates in particular populations, such as overweight persons or the elderly (9I). The costs of valid and reliable devices also limit applicability, as they are considerably higher compared to subjective measurements like questionnaires. Finally, a practical issue in using accelerometers is the necessity of expertise within the research team, which is essential to acquire complete measurements for accurate estimates of physical activity and sedentary time in daily life.

\section{METHODOLOGICAL CONSIDERATIONS}

\section{Setting}

Although the primary care setting has been advocated as the setting for overweight prevention and management (48-50), many lifestyle intervention studies were performed in controlled research setting and might suffer from selection bias and unrealistic execution (3I, 5I, II 5). In line with a recent review by Wadden et al. (48), we found that the primary care setting offers a feasible platform for effective lifestyle change in overweight people (CHAPTERS 5 and 6).

Social desirability might be an issue when researching an intervention in actual primary care. Participants might not be honest in reporting lifestyle behaviour to their health care provider. Hence, the questionnaires were provided and collected via Maastricht University post, which had a more neutral appearance to the participant. Notwithstanding, all clinical and functional outcomes (e.g. weight, blood pressure, aerobic capacity) were assessed by the HCPs in local primary care setting who also provided the lifestyle guidance (i.e. LSA 
and/or physiotherapist), possibly introducing bias in the measurements. This might have led to inflated results of the follow-up measurements in both programmes and in the supervised programme particularly.

\section{Design}

While the BeweegKuur trial was initially described as a clustered, multi-centre, randomised controlled trial (CHAPTER 4), the actual execution of the two programmes was not entirely according to protocol (CHAPTER 5). As discussed above, a certain amount of reinvention is necessary for long-term continuation of the programmes in primary care. In addition, this provides evidence for the effectiveness of programmes as they would be executed in actual care. On the other hand, this pragmatic design threatened the robustness of the findings in the trial. The re-invention introduced higher variability in programme execution, so identification of factors explaining the rejection of our hypothesis (difference between the programmes) is problematic. Nonetheless, the provided guidance in the two programmes was significantly different, so a difference in effects could still be expected. And more importantly, the results are a representation of what happened when the programmes were executed in daily primary care.

Traditionally, the control group would receive usual care. Our control group received the start-up programme, because the BeweegKuur trial was designed at the time that the government intended to reimburse the start-up programme in the Dutch basic health insurance, as an effective strategy to improve lifestyle and reduce overweight (63). Regardless of the issue whether or not the control condition was usual care at the moment of study, our design does not allow the conclusion that the changes in walking time, sitting time, dietary behaviour, weight, waist circumferences and health indica tors over time were caused by the BeweegKuur programmes. Therefore, the effects over time in the combined research population should be interpreted with caution.

The recruitment of participants in the start-up programme was considerably lower than in the supervised programme (i.e. 164 versus 247). HCPs from start-up and supervised locations stated similar reasons for low recruitment rates. Contamination between health care professionals and between participants could have been an explanation for the difference in recruitment numbers; however, we randomised entire health care clusters to either the start-up or the supervised programme to prevent this particular contamination effect. Nonetheless, all locations had the possibility to offer the supervised programme (the experimental programme) prior to the trial, which might have led to motivational issues in the HCPs in the control condition, as in their eyes they were not allowed to give sufficient guidance to their participants when the trial started. Consequently, recruitment 
may have hampered. Meanwhile, the effect of these motivational issues on programme execution appears minimal, as the dose delivered in control locations was more according to protocol than in experimental locations (CHAPTER 5). Nevertheless, care should be taken when evaluating an intervention which has already been implemented prior to the trial.

\section{Sample size}

A total of $22 \%$ of the participants did not complete the intervention period (not different between the two programmes). This is a good result in the light of earlier documented drop-out rates (II9), especially considering that the trial was set in actual primary care. The low drop-out rate probably played a role in the positive changes of the BeweegKuur participants over time.

The BeweegKuur trial encompassed cluster randomisation to minimise contamination between participants of different programmes and bias in guidance by HCPs. As a consequence, we had to apply an intra cluster correlation in the sample size calculation to account for the correlation of observations within clusters (222), the so-called design effect (223). The sample size calculation was published in a design article, and resulted in a target population size of 600 participants, i.e. 300 participants in each programme, i.e. 20 participants in each location (CHAPTER 4). Prior to study start, this recruitment goal was deemed feasible by both the developer of the BeweegKuur (NISB) and the primary care locations. Unfortunately, recruitment of participants proved to be more difficult than anticipated (CHAPTER 5). Firstly, recruitment in the two research arms was unbalanced, as discussed above. Secondly, not all primary care locations were able to recruit the agreed number of 20 participants and actual recruitment varied between two and 30 participants per location. Consequently, we increased cluster size variability by allowing locations with successful recruitment to recruit more than 20 participants. Both issues had consequences on the statistical power to detect differences between the two programmes (224). Eldridge et al. have argued that sample size calculations should account for cluster size variability when the ratio of the standard deviation of cluster size and the average cluster size is larger than 0.23 (224). Post hoc calculation reveals a coefficient of variation of cluster size in 0.63 in the current BeweegKuur trial (mean cluster size was I2.8 \pm 8.0 ), indicating that our initial sample size calculation might not have been adequate to detect our expected difference in MVPA. Recently, methods to account for the cluster size variability were published (225). Future trials should take the risk of cluster size variability into account, even if recruitment goals seem feasible. 
The number of follow-up measurements in the BeweegKuur trial $(59 \%$ of participants at I 2 months and $46 \%$ at 24 months) was lower than anticipated in the sample size calculation. The sample size calculation accounted for the drop-out of six locations (20 participants each) and $30 \%$ of participants, so a total number of 336 was required to detect a difference of 50 minutes of MVPA per week between the two programmes. Multilevel analyses were performed with 142 to 256 participants per analysis. These types of analyses use available data without imputation of missing data, preventing the introduction of uncertainty in the results caused by imputation (I78). Post-hoc calculations revealed small effect sizes, indicating that differences in MVPA between the programmes were trivial, supporting the conclusions based on the $p$-values of the multilevel analyses. A post hoc calculation concerning sample size showed that the acquired sample size with valid MVPA measurements (88 and 123 participants respectively in the start-up and supervised programme) should enable us to detect a difference of 172 weekly minutes of MVPA, i.e. 25 minutes MVPA per day, between the programmes. In the light of the high dose of guidance in the supervised programme, a difference of 172 minutes per week might not be unrealistic. In the first three months the programme consisted of two or three sessions per week with the physiotherapist, already accounting for 120 to 180 minutes of extra MVPA per week compared to the start-up programme. Besides these planned exercise sessions, participants were facilitated and motivated to adopt physical activities in their daily life, potentially increasing the number of extra minutes MVPA compared to participants in the start-up programme. Other studies also showed that participants have been able to increase physical activity time up to $150 \%$ of their baseline values in a similar intervention for people at high risk for diabetes (5I) and up to two hours after an intervention with Motivational Interviewing in primary care, but without group sessions (32). Nevertheless, the a priori expected difference was 50 minutes of MVPA, and to detect this difference we did not recruit enough participants. Therefore, differences caused by the additional guidance in the supervised programme might have remained undetected due to insufficient power.

\section{Outcome measures}

In our study we assessed physical activity and sitting time with the IPAQ short form (I26), which is easy to apply in large study samples. Like we mentioned earlier, accelerometry can provide a valid and reliable estimate of sedentary time and physical activity (CHAPTER 2 ), but practical issues might hinder implementation of accelerometry devices in largescale trials such as the BeweegKuur. The low number of accelerometry measurements and the inability to discriminate MVPA from physical activity at light intensity (due to reliability 
issues) forced us to use the IPAQ to estimate physical activity levels. Though this questionnaire has often been used in intervention trials, the IPAQ was developed as a surveillance tool for physical activity (126). The reliability of the IPAQ was argued to be good enough to be used as a repeated measure $(126,169)$, even though exact differences in PA might not be accurate (182). Hence, the differences in walking in the BeweegKuur might not be exact estimates, but we can conclude that walking time increased over time. Nonetheless, the IPAQ might not have been sensitive enough to detect potential differences between the programmes.

Dietary habits were assessed with a short questionnaire of 21 items, based on the validated Fat list (I27), which quantifies weekly consumption of products with an impact on health (e.g. fruit, snacks and candy). The psychometric properties of the short Fat questionnaire have not yet been assessed. A risk of over reporting of healthy behaviour and underreporting of unhealthy behaviour exists in subjective measurement of dietary behaviour. Even though we found changes in dietary habits, the responsivity, validity and reliability of the detected changes are unknown and remain to be assessed.

Physiological outcome measurements were executed by the participants' health care providers for practical reasons. This may have led to bias in the measurements. However, by standardizing the measurements, e.g. supplying all HCPs with similar weighing scales, we tried to minimise the effects of potential bias. In addition, questionnaires regarding the primary outcome, i.e. the IPAQ, were sent to participants directly with an envelope to return it directly to the researchers. Using this procedure, HCPs did not receive information about these results and we attempted to minimise effects of social desirability.

\section{IMPLICATIONS FOR RESEARCH}

The results of the studies described in this thesis, and their relationship with other research, has several implications for combined lifestyle interventions in overweight people, for the implementation in primary care and for the measurement of physical behaviour.

Participants in the BeweegKuur showed sustained changes in lifestyle, BMI, waist circumference and fat mass; however, of the cardiovascular risk profile parameters, only $\mathrm{HbAlc}$ and systolic blood pressure changed over time. In contrast, several studies in a dedicated research setting did find beneficial changes in HDL and triglyceride levels ( $3 \mathrm{I}$, $33,226)$, but these changes were accompanied by large weight loss which were not attained in studies in actual primary care $(30,32,36)$. Apolipoprotein B has been hypothesised to be a more sensitive marker for reduced cardiovascular health risk (227), 
and measurements of apolipoprotein B might therefore be advised to reveal beneficial changes in blood lipids caused by physical activity when applied in lifestyle trials.

Regarding primary care as a setting for treatment of overweight and obesity, we showed that the BeweegKuur was a feasible intervention. However, opportunity for re-invention was crucial. A large number of HCPs stated that the intervention was adapted to maintain feasibility in daily practice and to prevent participants from dropping out. But, like we already discussed, unrestricted re-invention might jeopardise the effectivity of intervention components. Therefore, future research should focus on the determination of the specific intervention aspects that are allowed and are not allowed to be re-invented, and on strategies to enhance feasibility and adherence in daily practice using this information.

Effective application of accelerometers as measuement and feedback devices for physical activity and sedentary behaviour needs further research and, above all, consensus. A wide range of activity monitors has been tested and applied, but with varying quality and a wide range of outcomes. This inconsistency limits comparability of studies and lifestyle interventions. On the other hand, different outcome parameters are necessary depending on the aim of particular interventions (e.g. physical activity intensity or sedentary time) and there is an urgent need for consensus regarding the criteria that should be used for each parameter. In addition, transparency in data treatment, by providing detailed information about the cut-off points, non-wear algorithm and epoch length, are crucial for the utilisation of existing knowledge in further research and real-life applications.

\section{IMPLICATIONS FOR CLINICAL PRACTICE}

Because the BeweegKuur trial was embedded in primary care, we can give suggestions for the further development, implementation and continuation of combined lifestyle interventions in this setting.

Although our primary outcome, MVPA, did not change significantly, beneficial changes were observed in walking, weight, waist circumference and some cardiovascular risk factors. Both programmes can be used to reduce health risk in people with overweight and comorbidities or with obesity. However, the extra number of group meetings with the physiotherapist of the supervised programme, did not yield additional benefits compared to the start-up programme that had a lower dose of these meetings. Also, feasibility of the supervised programme was lower than that of the start-up programme and cost-effectiveness results of the supervised programme are not yet published. Thus, we advise to include combined lifestyle interventions like the start-up programme as usual 
care for the overweight and obese population in the primary health care in the Netherlands.

Several promoting and hindering aspects were identified for long-term continuation of lifestyle interventions like the BeweegKuur in primary care. Firstly, allowing for re-invention is essential for feasibility and continuation. HCPs should be able to adjust aspects of the intervention so they remain capable and motivated to provide the intervention, and to prevent drop-out of participants. In daily practice, the BeweegKuur should not be viewed as a strict protocol, but more as a guideline for HCPs under the condition that vital elements of the intervention are maintained. Key elements of the BeweegKuur were described earlier $(56,57)$, and include individual meetings with Motivational Interviewing aimed at both diet and physical activity. Further, in most participating practices one of the HCPs played a key role in the organisation and continuation of the intervention. Therefore, care should be taken to enable and motivate this key figure to invest time in the intervention. Unfortunately, the Achilles heel of this approach is that once this HCP leaves the primary care practice, continuation of the programme is at risk. Finally, proper and reliable funding is crucial for long-term continuation of an intervention. Projects should include a clear funding plan, to ensure that HCPs are enabled to invest their time in the intervention, as discussed above. The lesson learned from our BeweegKuur trial is that the implementation of combined lifestyle interventions is sensitive to political changes, and potential future reimbursement does not imply permanent funding for the programmes.

Accelerometers can be applied in primary care setting to provide insight for HCPs into physical activity behaviour of patients to tailor their counselling. Although feedback about physical activity was not integrated in the studies of this thesis, our research does provide some implications for this type of prevention. Accelerometers are not always thoroughly tested, and we advise to only use devices that are user friendly and provide valid and reliable estimates for the outcome of interest. In addition, the impact of decisions within the data-handling is not always evident, especially for HCPs with no technical background. Thus, accelerometers and their output should be comprehensible for the layman in order to work as feedback tool, and should therefore be specifically designed for this means. For example, van der Weegen et al. have successfully developed a feedback tool with accelerometry using a user-centred design (228). 


\section{CONCLUSION}

The research described in this thesis indicated that additional group meetings aimed at physical activity in a combined lifestyle intervention, which already includes guidance by LSA, physiotherapist and dietician, did not lead to additional effects in participants with overweight or obesity. Nevertheless, sustained improvements in lifestyle and health were observed in participants in both the start-up and supervised programme. Because the trial was set in actual primary care practices, re-invention by HCPs occurred and the execution of the intervention was not according to protocol. And although HCPs reduced the number of meetings to maintain feasibility, absence of funding hindered the continuation of the intervention in some primary care practices. The measurement of physical activity by means of accelerometry is promising, but application in a large-scale, multi-centre trial is challenging. Lack of a valid cut off point for MVPA and the low number of complete measurements restricted the usability of these data. Although the application of accelerometers has some limitations, tri-axial thigh-worn devices can provide valid estimates of physical activity and sitting time. 



\section{Valorisation}


The prevalence of obesity is increasing and is becoming a major health problem because excess body weight leads to increased morbidity, health care costs and mortality $(1,2,4)$. Moreover, a lack of physical activity is not merely a cause of overweight, it also influences cardio-metabolic risk directly $(5,6)$. Targeting interventions at excess body weight via physical activity (and nutritional) behaviour might therefore improve health collectively. In addition, psychosocial health might also improve, as both body weight and physical activity are related to quality of life $(28,108)$.

The current thesis described research into the measurement of physical activity behaviour and the promotion of physical activity. The first two studies looked into the measurement of physical activity (CHAPTERS 2 and 3); the other studies concerned the process and effect evaluation of a multidisciplinary lifestyle intervention (the BeweegKuur) promoting physical activity behaviour and healthy nutrition (CHAPTERS 4, 5 and 6). There is great potential for the integration of the measurement and the promotion of physical activity in research and in daily practice.

Research has focused on the application of activity monitors for the description of physical activity levels of a population or group, or as a tool to assess change in physical activity in the search for effective methods to improve lifestyle (66). Recently, researchers and companies involved in physical activity monitoring are more dedicated to using all information that activity monitors provide (e.g. the Fitbit, the Philips DirectLife and the ActiGraph Link).

Lifestyle coaching has often been proved to be effective, in particular when based on behaviour change techniquesbut the effects of these interventions are not always maintained in the long run. The effectiveness evaluation of the BeweegKuur programmes in chapter 6 suggested that one year of intensive counselling improved lifestyle and several cardio-metabolic risk factors. Beneficial changes in lifestyle and body composition were even maintained during the second year of study, after a year without BeweegKuur counselling. However, at the two year follow-up measurement, HbAlc and systolic blood pressure were similar to baseline levels, suggesting that sustained counselling might be necessary. Although monthly counselling sessions might ensure maintenance of effects on cardio-metabolic risk factors (34) with the expected long term decrease in health care use, the direct health care costs of such guidance oppose the implementation.

Self-management provides a valuable method to maintain physical activity level, without relying on long term counselling in health care. Activity monitors can support selfmanagement by providing feedback about physical activity level to the user. As described in chapter 2, activity monitors offer a user friendly method to acquire valid and reliable estimates of physical (in)activity. Moreover, activity monitors can estimate sedentary 
behaviour based on the orientation of the device, when placed on a suitable place on the human body. By using an activity monitor which is capable to estimate physical activity and sedentary time in a user friendly way, both these health-threatening behaviours can be targeted. Self-report leads to both overestimations and underestimations of physical activity behaviour (74), so awareness about people's actual physical (in)activity is vital to inform them about (potential for) improvements. Nowadays, several devices and smart phone apps have been developed and marketed to this end and they are often combined with aspects of established behaviour change techniques. Nevertheless, there is a lack of evidence of the effectiveness of this approach $(229,230)$, and self-monitoring is suggested to be effective especially when combined with other techniques for behaviour change (231). A viable, promising solution would be to combine the feedback from valid and user friendly activity monitors with the lifestyle counselling that is firmly based in behaviour change techniques. This combination would benefit from the extensive research into determinants influencing behavior (e.g. self-efficacy, motivation, social support) with the long term continuity by means of activity monitoring and regulating a healthy lifestyle. $A$ study concerning a self-management programme with four meetings with practice nurse and a feedback device for physical activity showed improved physical activity level after three months (22I). It might be hypothesized that the strategies used in the BeweegKuur (i.e. Motivational Interviewing and multidisciplinary guidance) can enlarge the effects of the feedback. Vice versa, providing feedback about physical activity level to participants of the BeweegKuur might enhance potential changes in behaviour.

Previous research has shown that multidisciplinary lifestyle interventions based on behaviour change techniques are effective $(33,34,42,188,192)$. The process and effect evaluation of the BeweegKuur in chapter 4 and 5 have reinforced the decision to classify the start-up and supervised programmes as 'first signs for effectiveness' by the Dutch Loketgezondleven.nl, an initiative by the ministry of Health, Welfare and Sports to inform professionals about the quality of health promotion programmes (232). Informing professionals about the evidence for the BeweegKuur stimulates implementation of the intervention in daily practice.

The interviews with health care providers of the process evaluation in chapter 5 revealed a crucial role of the governmental changes in the discontinuity of the BeweegKuur after our study. It appeared that primary health care lacks the agility to respond adequately to changes in the reimbursement of costs, which were greatly determined by the governmental agenda. In the midst of the design and execution of the BeweegKuur study, the Dutch government changed. The focus within health care shifted from a government intending to take care of everything from cradle to grave towards a government 
stimulating people's own responsibility (I53). Moreover, the financial crisis of 2008 led to financial cutbacks, also within the health care system. Many of the health care practices participating in the BeweegKuur study did not anticipate or were not able to anticipate on the consequences of this change (i.e. no reimbursement for lifestyle interventions). Although many primary care practices stated to have faced problems in the implementation and/or continuation of the BeweegKuur, some have set a good example for the embedding of combined lifestyle interventions. Reimbursement of the intervention ceased, but several professionals found a way to continue this care to overweight people in their location. Successful practices either invested time in agenda-setting with health care insurances or embarked on extensive collaborations (between care providers and other organisations such as municipality). Perseverance, dedication and time investment proved to be the key to success. Therefore, investments in key professionals, i.e. lifestyle advisors, are essential to continuity of lifestyle counselling.

The evaluation of the BeweegKuur programmes in chapter 6 reinforces the earlier results suggesting that the multidisciplinary lifestyle guidance improves physical activity level. As described in chapter 5 , health care professionals value multidisciplinary lifestyle guidance, and with sufficient investments, the implementation and long term delivery of the programmes might be ensured. In addition, the study in chapter 2 has informed researchers and practitioners about the validity and user friendliness of several activity monitors that are commercially available. Combining this knowledge offers relevant opportunities for lifestyle improvement in overweight and obese adults in health care. 
References 
I. Lim SS, Vos T, Flaxman AD, Danaei G, Shibuya K, Adair-Rohani H, et al. A comparative risk assessment of burden of disease and injury attributable to 67 risk factors and risk factor clusters in 21 regions, 1990-2010: a systematic analysis for the Global Burden of Disease Study 20 I0. Lancet. 2012 Dec 15;380(9859):2224-60.

2. Hu F. Obesity Epidemiology. New York: Oxford University Press, Inc; 2008.

3. CBS. Zelfgerapporteerde leefstijl. [Self-reported lifestyle]. Statistics Netherlands 2015 [updated 20I5; cited 2015 March 3,]. Available from: http://statline.cbs.nl.

4. in 't Panhuis - Plasmans M, Luijben G, Hoogenveen R. Zorgkosten van ongezond gedrag [Health care costs of unhealthy behaviour]. Rijksinstituut voor Volksgezondheid en Milieu (RIVM). 2012.

5. Ekelund U, Franks PW, Sharp S, Brage S, Wareham NJ. Increase in physical activity energy expenditure is associated with reduced metabolic risk independent of change in fatness and fitness. Diabetes Care. 2007 Aug;30(8):21 01 -6.

6. McAuley PA, Blair SN. Obesity paradoxes. J Sports Sci. 20II May;29(8):773 -82.

7. Herzig $\mathrm{KH}$, Ahola R, Leppaluoto J, Jokelainen J, Jamsa T, Keinanen-Kiukaanniemi S. Light physical activity determined by a motion sensor decreases insulin resistance, improves lipid homeostasis and reduces visceral fat in high-risk subjects: PreDiabEx study RCT. International journal of obesity. 20I4 Aug;38(8):1089-96.

8. Archer E, Blair SN. Physical activity and the prevention of cardiovascular disease: from evolution to epidemiology. Progress in cardiovascular diseases. 20 II MayJun;53(6):387-96.

9. Peddie MC, Bone JL, Rehrer NJ, Skeaff CM, Gray AR, Perry TL. Breaking prolonged sitting reduces postprandial glycemia in healthy, normal-weight adults: a randomized crossover trial. The American journal of clinical nutrition. 20 I 3 Aug;98(2):358-66.

10. Latouche C, Jowett JB, Carey AL, Bertovic DA, Owen N, Dunstan DW, et al. Effects of breaking up prolonged sitting on skeletal muscle gene expression. Journal of applied physiology. 2013 Feb I 5; I 14(4):453-60.

II. van der Ploeg HP, Chey T, Korda RJ, Banks E, Bauman A. Sitting time and all-cause mortality risk in 222497 Australian adults. Arch Intern Med. 2012 Mar 26; I 72(6):494-500.

12. Bankoski A, Harris TB, McClain JJ, Brychta RJ, Caserotti P, Chen KY, et al. Sedentary activity associated with metabolic syndrome independent of physical activity. Diabetes Care. 20I I Feb;34(2):497-503.

13. Hamilton MT, Hamilton DG, Zderic TW. Role of low energy expenditure and sitting in obesity, metabolic syndrome, type 2 diabetes, and cardiovascular disease. Diabetes. 2007 Nov;56(I I):2655-67.

14. Stevens JW, Khunti K, Harvey R, Johnson M, Preston L, Woods HB, et al. Preventing the progression to type 2 diabetes mellitus in adults at high risk: a systematic review and network meta-analysis of lifestyle, pharmacological and surgical interventions. Diabetes research and clinical practice. 2015 Mar;I07(3):320-3I.

15. Chang SH, Stoll CR, Song J, Varela JE, Eagon CJ, Colditz GA. The effectiveness and risks of bariatric surgery: an updated systematic review and meta-analysis, 2003-20I2. JAMA surgery. 2014 Mar; I 49(3):275-87.

16. Kumar RB, Aronne LJ. Efficacy comparison of medications approved for chronic weight management. Obesity (Silver Spring). 2015 Apr;23 Suppl I:S4-7.

17. Sackner-Bernstein J, Kanter D, Kaul S. Dietary Intervention for Overweight and Obese Adults: Comparison of Low-Carbohydrate and Low-Fat Diets. A MetaAnalysis. PloS one. 20I5; I0(I0):e01398I7. 
18. Woodward-Lopez G, Kao J, Ritchie L. To what extent have sweetened beverages contributed to the obesity epidemic? Public Health Nutr. 20 I I Mar; I 4(3):499-509.

19. Bes-Rastrollo M, Schulze MB, Ruiz-Canela M, Martinez-Gonzalez MA. Financial Conflicts of Interest and Reporting Bias Regarding the Association between SugarSweetened Beverages and Weight Gain: A Systematic Review of Systematic Reviews. PLoS medicine. 20I3 Dec; I0(I2).

20. DiNicolantonio JJ, Lucan SC. The wrong white crystals: not salt but sugar as aetiological in hypertension and cardiometabolic disease. Open heart. 20I4; I (I):e000I67.

21. Wang X, Ouyang Y, Liu J, Zhu M, Zhao G, Bao W, et al. Fruit and vegetable consumption and mortality from all causes, cardiovascular disease, and cancer: systematic review and dose-response meta-analysis of prospective cohort studies. BMJ. 20। 4;349:g4490.

22. Saha S, Gerdtham UG, Johansson P. Economic evaluation of lifestyle interventions for preventing diabetes and cardiovascular diseases. International journal of environmental research and public health. 2010 Aug;7(8):3I50-95.

23. Brunner E, Cohen D, Toon L. Cost effectiveness of cardiovascular disease prevention strategies: a perspective on EU food based dietary guidelines. Public Health Nutr. 200 I Apr;4(2B):7 I I-5.

24. Dalziel K, Segal L. Time to give nutrition interventions a higher profile: costeffectiveness of 10 nutrition interventions. Health promotion international. 2007 Dec;22(4):27 I -83.

25. Hawley JA, Gibala MJ. What's new since Hippocrates? Preventing type 2 diabetes by physical exercise and diet. Diabetologia. 2012 Mar;55(3):535-9.

26. Vissers D, Hens W, Taeymans J, Baeyens JP, Poortmans J, Van Gaal L. The Effect of Exercise on Visceral Adipose Tissue in Overweight Adults: A Systematic Review and Meta-Analysis. PloS one. 2013 Feb 8;8(2).

27. Duvivier BM, Schaper NC, Bremers MA, van Crombrugge G, Menheere PP, Kars M, et al. Minimal intensity physical activity (standing and walking) of longer duration improves insulin action and plasma lipids more than shorter periods of moderate to vigorous exercise (cycling) in sedentary subjects when energy expenditure is comparable. PloS one. 2013;8(2):e55542.

28. Sorensen J, Sorensen JB, Skovgaard T, Bredahl T, Puggaard L. Exercise on prescription: changes in physical activity and health-related quality of life in five Danish programmes. Eur J Public Health. 20I I Feb;2 I (I):56-62.

29. Cobiac LJ, Vos T, Barendregt JJ. Cost-effectiveness of interventions to promote physical activity: a modelling study. PLoS medicine. 2009 Jul I4;6(7):el 000 I I 0.

30. Driehuis F, Barte JC, Ter Bogt NC, Beltman FW, Smit AJ, van der Meer K, et al. Maintenance of lifestyle changes: 3-year results of the Groningen Overweight and Lifestyle study. Patient education and counseling. 20 I 2 Aug;88(2):249-55.

3I. Goodpaster BH, Delany JP, Otto AD, Kuller L, Vockley J, South-Paul JE, et al. Effects of diet and physical activity interventions on weight loss and cardiometabolic risk factors in severely obese adults: a randomized trial. Jama. 2010 Oct 27;304(I 6): I 795 802.

32. Hardcastle S, Taylor A, Bailey M, Castle R. A randomised controlled trial on the effectiveness of a primary health care based counselling intervention on physical activity, diet and CHD risk factors. Patient education and counseling. 2008 Jan;70(I):3 I -9. 
33. Look Ahead Research Group. Long-term effects of a lifestyle intervention on weight and cardiovascular risk factors in individuals with type 2 diabetes mellitus: four-year results of the Look AHEAD trial. Arch Intern Med. 2010 Sep 27;170(I7):I 566-75.

34. Look Ahead Research Group. Eight-year weight losses with an intensive lifestyle intervention: the look AHEAD study. Obesity (Silver Spring). 2014 Jan;22(I):5-I 3.

35. Mensink M, Blaak EE, Corpeleijn E, Saris WH, de Bruin TW, Feskens EJ. Lifestyle intervention according to general recommendations improves glucose tolerance. Obes Res. 2003 Dec; I I (I 2): I 588-96.

36. Vermunt PW, Milder IE, Wielaard F, de Vries JH, Baan CA, van Oers JA, et al. A lifestyle intervention to reduce Type 2 diabetes risk in Dutch primary care: 2.5 -year results of a randomized controlled trial. Diabetic medicine : a journal of the British Diabetic Association. 20I2 Aug;29(8):e223-3I.

37. Mastellos N, Gunn LH, Felix LM, Car J, Majeed A. Transtheoretical model stages of change for dietary and physical exercise modification in weight loss management for overweight and obese adults. The Cochrane database of systematic reviews. 20। 4;2:CD008066.

38. Look Ahead Research Group, Wadden TA, West DS, Delahanty L, Jakicic J, Rejeski J, et al. The Look AHEAD study: a description of the lifestyle intervention and the evidence supporting it. Obesity (Silver Spring). 2006 May; I 4(5):737-52.

39. Breyer BN, Phelan S, Hogan PE, Rosen RC, Kitabchi AE, Wing RR, et al. Intensive lifestyle intervention reduces urinary incontinence in overweight/obese men with type 2 diabetes: results from the Look AHEAD trial. The Journal of urology. 20I4 Jul; I 92(I):। 44-9.

40. Rejeski WJ, Ip EH, Bertoni AG, Bray GA, Evans G, Gregg EW, et al. Lifestyle change and mobility in obese adults with type 2 diabetes. The New England journal of medicine. 20I 2 Mar 29;366(I3):I209-I7.

4I. Espeland MA, Glick HA, Bertoni A, Brancati FL, Bray GA, Clark JM, et al. Impact of an Intensive Lifestyle Intervention on Use and Cost of Medical Services Among Overweight and Obese Adults With Type 2 Diabetes: The Action for Health in Diabetes. Diabetes Care. 20I4 Sep;37(9):2548-56.

42. Wadden TA, Grp LAR. Impact of Intensive Lifestyle Intervention on Depression and Health-Related Quality of Life in Type 2 Diabetes: The Look AHEAD Trial. Diabetes Care. 2014 Jun;37(6): I 544-53.

43. Look Ahead Research Group, Wing RR, Bolin P, Brancati FL, Bray GA, Clark JM, et al. Cardiovascular effects of intensive lifestyle intervention in type 2 diabetes. The New England journal of medicine. 20I3 Jul I I;369(2): |45-54.

44. Jacobs-van der Bruggen MA, van Baal PH, Hoogenveen RT, Feenstra TL, Briggs AH, Lawson K, et al. Cost-effectiveness of lifestyle modification in diabetic patients. Diabetes Care. 2009 Aug;32(8): I 453-8.

45. Li R, Zhang P, Barker LE, Chowdhury FM, Zhang X. Cost-effectiveness of interventions to prevent and control diabetes mellitus: a systematic review. Diabetes Care. 2010 Aug;33(8): I872-94.

46. The Netherlands National Contact Point of the National Health Care Institute. How is health care organised in the Netherlands? 2015 [cited 2015 November 27,].

47. Faber MJ, Burgers JS, Westert GP. A sustainable primary care system: lessons from the Netherlands. The Journal of ambulatory care management. 2012 JulSep;35(3): 174-8I. 
48. Wadden TA, Butryn ML, Hong PS, Tsai AG. Behavioral treatment of obesity in patients encountered in primary care settings: a systematic review. Jama. 2014 Nov 5;3। 2(I7): I779-9I.

49. Pettigrewe LM, De Maeseneer J, Anderson MP, Essuman A, Kidd MR, Haines A. Primary health care and the Sustainable Development Goals. The Lancet. 2015;Volume 386(No. 10009):p2 II9-2I.

50. Visser F, Hiddink G, Koelen M, van Binsbergen J, Tobi H, van Woerkum C. Longitudinal changes in GPs' task perceptions, self-efficacy, barriers and practices of nutrition education and treatment of overweight. Fam Pract. 2008 Dec;25 Suppl I:il05-II.

5I. Knowler WC, Barrett-Connor E, Fowler SE, Hamman RF, Lachin JM, Walker EA, et al. Reduction in the incidence of type 2 diabetes with lifestyle intervention or metformin. The New England journal of medicine. 2002 Feb 7;346(6):393 -403.

52. Green LW. Making research relevant: if it is an evidence-based practice, where's the practice-based evidence? Family Practice. 2008 Dec;25:120-14.

53. Paulussen T, Wiefferink K, Mesters I. Invoering van effectief gebleken interventies. Gezondheidsvoorlichting en bevordering2007. p. I5I -72.

54. Linmans JJ, Spigt MG, Deneer L, Lucas AE, de Bakker M, Gidding LG, et al. Effect of lifestyle intervention for people with diabetes or prediabetes in real-world primary care: propensity score analysis. BMC family practice. 2011 ; I 2:95.

55. Hartmann-Boyce J, Johns DJ, Jebb SA, Summerbell C, Aveyard P, Grp BWMR. Behavioural weight management programmes for adults assessed by trials conducted in everyday contexts: systematic review and meta-analysis. Obesity Reviews. 2014 Nov; I 5 (I I ):920-32.

56. De Weerdt I, Broeders I, Schaars D. Prototype De BeweegKuur. Het beste recept voor uw gezondheid. Een interventie voor de (eerstelijns) zorg om mensen met (een hoog risico op) diabetes mellitus type 2 te begeleiden naar een actievere leefstijl [Prototype The BeweegKuur. The best recipe for your health. An intervention for (primary health) care to guide people with (an increased risk for) type 2 diabetes mellitus to a more active lifestyle]. NISB. 2008.

57. Helmink JH, Meis JJ, de Weerdt I, Visser FN, de Vries NK, Kremers SP. Development and implementation of a lifestyle intervention to promote physical activity and healthy diet in the Dutch general practice setting: the BeweegKuur programme. Int J Behav Nutr Phys Act. 2010;7:49.

58. Deci EL, Ryan RM. The "what" and "why" of goal pursuits: Human needs and the selfdetermination of behavior. Psychol Inq. 2000; I I (4):227-68.

59. Teixeira PJ, Silva MN, Mata J, Palmeira AL, Markland D. Motivation, selfdetermination, and long-term weight control. Int J Behav Nutr Phys Act. 201 2;9:22.

60. Rutten GM, Meis J, Hendriks M, Hamers F, Veenhof C, Kremers S. The contribution of lifestyle coaching of overweight patients in primary care to more autonomous motivation for physical activity and healthy dietary behaviour: results of a longitudinal study. Int J Behav Nutr Phys Act. 20 I 4 Jul I6; I I (I):86.

6I. Kwak L, Kremers SPJ, Brug J, Walsh A. How is your walking group running? Health Education. 2006;106(I):2I-3I.

62. Fraser SN, Spink KS. Examining the role of social support and group cohesion in exercise compliance. Journal of behavioral medicine. 2002 Jun;25(3):233-49.

63. Bemelmans WJE, Wendel-Vos W, Bogers RP, Milder IE, de Hollander EL, Barte JCM, et al. (Kosten)effectiviteit beweeg- en dieetadvisering bij mensen met (hoog risico 
op) diabetes mellitus type 2: Literatuuronderzoek en modelsimulaties rondom de Beweegkuur [(Cost-)effectiveness of advice on physical activity and diet in persons with (an increased risk of) type 2 diabetes mellitus: Literature study and model simulations of the BeweegKuur]. Rijksinstituut voor Volksgezondheid en Milieu (RIVM). 2008.

64. College voor Zorgverzekeringen. De gecombineerde leefstijlinterventie: kosten, opbrengsten en de praktijk [Combined lifestyle interventions: costs, benefits and practice]. Diemen: 2010.

65. Corder K, Brage S, Ekelund U. Accelerometers and pedometers: methodology and clinical application. Curr Opin Clin Nutr Metab Care. 2007 Sep; I 0(5):597-603.

66. Mathie MJ, Coster AC, Lovell NH, Celler BG. Accelerometry: providing an integrated, practical method for long-term, ambulatory monitoring of human movement. Physiol Meas. 2004 Apr;25(2):RI -20.

67. Janssen $X$, Cliff DP. Issues related to measuring and interpreting objectively measured sedentary behavior data. Measurement in Physical Education and Exercise Science. 2015;19(3): I 16-24.

68. Trost SG, Mclver KL, Pate RR. Conducting accelerometer-based activity assessments in field-based research. Med Sci Sports Exerc. 2005 Nov;37(I I Suppl):S53 I -43.

69. Choi L, Ward SC, Schnelle JF, Buchowski MS. Assessment of Wear/Nonwear Time Classification Algorithms for Triaxial Accelerometer. Med Sci Sport Exer. 2012 Apr 19.

70. World Health Organization. Global health risks: mortality and burden of disease attributable to selected major risks. Geneva, Switzerland: WHO Press, 2009.

7I. Clark BK, Sugiyama T, Healy GN, Salmon J, Dunstan DW, Owen N. Validity and reliability of measures of television viewing time and other non-occupational sedentary behaviour of adults: a review. Obes Rev. 2009 Jan; I0(I):7-I6.

72. Healy GN, Clark BK, Winkler EA, Gardiner PA, Brown WJ, Matthews CE. Measurement of adults' sedentary time in population-based studies. Am J Prev Med. 20I I Aug;4 I (2):2 I 6-27.

73. Thorp AA, Owen N, Neuhaus M, Dunstan DW. Sedentary behaviors and subsequent health outcomes in adults a systematic review of longitudinal studies, 1996-201 I. Am J Prev Med. 20I I Aug;4I(2):207-I5.

74. Prince SA, Adamo KB, Hamel ME, Hardt J, Connor Gorber S, Tremblay M. A comparison of direct versus self-report measures for assessing physical activity in adults: a systematic review. Int J Behav Nutr Phys Act. 2008;5:56.

75. Foerster F, Fahrenberg J. Motion pattern and posture: correctly assessed by calibrated accelerometers. Behav Res Methods Instrum Comput. 2000 Aug;32(3):450-7.

76. Oliver M, Schofield GM, Badland HM, Shepherd J. Utility of accelerometer thresholds for classifying sitting in office workers. Prev Med. 2010 Nov;5 I (5):357-60.

77. Carr LJ, Mahar MT. Accuracy of intensity and inclinometer output of three activity monitors for identification of sedentary behavior and light-intensity activity. J Obes. 2012;20 I 2:Article ID 46027I, 9 pages.

78. Kozey-Keadle S, Libertine A, Lyden K, Staudenmayer J, Freedson PS. Validation of wearable monitors for assessing sedentary behavior. Med Sci Sports Exerc. 201I Aug;43(8): I 56 I -7. 
79. Freedson P, Bowles HR, Troiano R, Haskell W. Assessment of physical activity using wearable monitors: recommendations for monitor calibration and use in the field. Med Sci Sport Exer. 2012 Jan;44(I Suppl I):SI -4.

80. Kozey-Keadle S, Libertine A, Staudenmayer J, Freedson P. The Feasibility of Reducing and Measuring Sedentary Time among Overweight, Non-Exercising Office Workers. J Obes. 20I2;20I 2:282303.

8I. Annegarn J, Spruit MA, Uszko-Lencer NH, Vanbelle S, Savelberg HH, Schols AM, et al. Objective physical activity assessment in patients with chronic organ failure: a validation study of a new single-unit activity monitor. Arch Phys Med Rehabil. 20 II Nov;92(I I): I 852-7 el.

82. McMahon GC, Brychta RJ, Chen KY. Validation of the Actigraph (GT3X) inclinometer function. Medicine \& Science in Sports \& Exercise. 20 I 0;42(5):489.

83. Godfrey A, Culhane KM, Lyons GM. Comparison of the performance of the activPAL Professional physical activity logger to a discrete accelerometer-based activity monitor. Med Eng Phys. 2007 Oct;29(8):930-4.

84. Grant PM, Ryan CG, Tigbe WW, Granat $M H$. The validation of a novel activity monitor in the measurement of posture and motion during everyday activities. $\mathrm{Br} J$ Sports Med. 2006 Dec;40(I2):992-7.

85. Hart TL, Ainsworth BE, Tudor-Locke C. Objective and subjective measures of sedentary behavior and physical activity. Med Sci Sports Exerc. 20I I Mar;43(3):449 56.

86. Landis JR, Koch GG. The measurement of observer agreement for categorical data. Biometrics. 1977 Mar;33(I):I59-74.

87. Maddocks M, Petrou A, Skipper L, Wilcock A. Validity of three accelerometers during treadmill walking and motor vehicle travel. $\mathrm{Br} J$ Sports Med. 2010 Jun;44(8):606-8.

88. Dahlgren G, Carlsson D, Moorhead A, Hager-Ross C, McDonough SM. Test-retest reliability of step counts with the ActivPAL device in common daily activities. Gait Posture. 2010 Jul;32(3):386-90.

89. Bouten CV, Sauren AA, Verduin M, Janssen JD. Effects of placement and orientation of body-fixed accelerometers on the assessment of energy expenditure during walking. Med Biol Eng Comput. 1997 Jan;35(I):50-6.

90. Troiano RP, Berrigan D, Dodd KW, Masse LC, Tilert T, McDowell M. Physical activity in the United States measured by accelerometer. Med Sci Sports Exerc. 2008 Jan;40(I): I 8 I -8.

91. Winkler EA, Gardiner PA, Clark BK, Matthews CE, Owen N, Healy GN. Identifying sedentary time using automated estimates of accelerometer wear time. Br J Sports Med. 2012 May;46(6):436-42.

92. Machado-Rodrigues AM, Figueiredo AJ, Mota J, Cumming SP, Eisenmann JC, Malina RM, et al. Concurrent validation of estimated activity energy expenditure using a 3 day diary and accelerometry in adolescents. Scand J Med Sci Sports. 2012 Apr;22(2):259-64.

93. Tudor-Locke C, Brashear MM, Johnson WD, Katzmarzyk PT. Accelerometer profiles of physical activity and inactivity in normal weight, overweight, and obese U.S. men and women. Int J Behav Nutr Phys Act. 2010;7:60.

94. Evenson KR, Terry JW, Jr. Assessment of differing definitions of accelerometer nonwear time. Res Q Exerc Sport. 2009 Jun;80(2):355-62. 
95. Healy GN, Dunstan DW, Salmon J, Cerin E, Shaw JE, Zimmet PZ, et al. Breaks in sedentary time: beneficial associations with metabolic risk. Diabetes Care. 2008 Apr;3 I (4):66 I -6.

96. Masse LC, Fuemmeler BF, Anderson CB, Matthews CE, Trost SG, Catellier DJ, et al. Accelerometer data reduction: a comparison of four reduction algorithms on select outcome variables. Med Sci Sports Exerc. 2005 Nov;37(I I Suppl):S544-54.

97. Miller GD, Jakicic JM, Rejeski WJ, Whit-Glover MC, Lang W, Walkup MP, et al. Effect of varying accelerometry criteria on physical activity: the look ahead study. Obesity (Silver Spring). 2013 Jan;2 I ( I):32-44.

98. Van Domelen DR, Koster A, Harris TB. Accelerometer nonwear algorithms: optimizing parameters for both wear states. Med Sci Sport Exer. 20 I I May;43(5):932; author reply 3 .

99. Choi L, Liu Z, Matthews CE, Buchowski MS. Validation of accelerometer wear and nonwear time classification algorithm. Med Sci Sports Exerc. 20I I Feb;43(2):357-64.

100. Harrington DM, Dowd KP, Bourke AK, Donnelly AE. Cross-sectional analysis of levels and patterns of objectively measured sedentary time in adolescent females. Int J Behav Nutr Phys Act. 20 I I;8:I 20.

I0I. Berendsen BA, Hendriks MR, Verhagen EA, Schaper NC, Kremers SP, Savelberg HH. Effectiveness and cost-effectiveness of 'BeweegKuur', a combined lifestyle intervention in the Netherlands: rationale, design and methods of a randomized controlled trial. BMC public health. 201।;1।:8I5.

102. Berendsen BA, Hendriks MR, Meijer K, Plasqui G, Schaper NC, Savelberg HH. Which activity monitor to use? Validity, reproducibility and user friendliness of three activity monitors. BMC public health. 20। 4; I4:749.

103. Hutto B, Howard VJ, Blair SN, Colabianchi N, Vena JE, Rhodes D, et al. Identifying accelerometer nonwear and wear time in older adults. Int J Behav Nutr Phys Act. 2013 Oct 25;10(I): 120.

104. Rosenberger ME, Haskell WL, Albinali F, Mota S, Nawyn J, Intille S. Estimating activity and sedentary behavior from an accelerometer on the hip or wrist. Med Sci Sports Exerc. 2013 May;45(5):964-75.

105. Pat Rapp M, Nelson F, Oliver M, Bergstrom N, Cron SG. Comparison of commonly used placement sites for activity monitoring. Biological research for nursing. 2010 Jan; I I (3):302-9.

106. Zhang S, Rowlands AV, Murray P, Hurst TL. Physical activity classification using the GENEA wrist-worn accelerometer. Med Sci Sports Exerc. 2012 Apr;44(4):742-8.

107. World Health Organization. Obesity: preventing and managing the global epidemic. Report of a WHO consultation. World Health Organ Tech Rep Ser. 2000;894:i-xii, I-253.

108. Blissmer B, Riebe D, Dye G, Ruggiero L, Greene G, Caldwell M. Health-related quality of life following a clinical weight loss intervention among overweight and obese adults: intervention and 24 month follow-up effects. Health Qual Life Outcomes. 2006;4:43.

109. CBS. Zelfgerapporteerde leefstijl. [Self-reported lifestyle]. Statistics Netherlands 2010 [updated 20I0; cited 2010 September I,]. Available from: http://statline.cbs.nI.

I I0. Polder JJ, Takken J, Meerding WJ, Kommer GJ, Stokx LJ. Kosten van ziekten in Nederland: De zorgeuro ontrafeld [Costs of diseases in the Netherlands]. Rijksinstituut voor Volksgezondheid en Milieu (RIVM). 2002. 
I I I. Cecchini M, Sassi F, Lauer JA, Lee YY, Guajardo-Barron V, Chisholm D. Tackling of unhealthy diets, physical inactivity, and obesity: health effects and cost-effectiveness. Lancet. 2010 Nov 20;376(9754): 1775-84.

I I 2. Jakicic JM. The effect of physical activity on body weight. Obesity (Silver Spring). 2009 Dec; 17 Suppl 3:S34-8.

I I3. Sullivan PW, Morrato EH, Ghushchyan V, Wyatt HR, Hill JO. Obesity, inactivity, and the prevalence of diabetes and diabetes-related cardiovascular comorbidities in the U.S., 2000-2002. Diabetes Care. 2005 Jul;28(7):I 599-603.

II4. Mensink M, Feskens EJ, Saris WH, De Bruin TW, Blaak EE. Study on Lifestyle Intervention and Impaired Glucose Tolerance Maastricht (SLIM): preliminary results after one year. Int J Obes Relat Metab Disord. 2003 Mar;27(3):377-84.

I I5. Tuomilehto J, Lindstrom J, Eriksson JG, Valle TT, Hamalainen H, llann e-Parikka P, et al. Prevention of type 2 diabetes mellitus by changes in lifestyle among subjects with impaired glucose tolerance. The New England journal of medicine. 200 I May 3;344(I 8): I 343-50.

I 16. Praet SF, van Rooij ES, Wijtvliet A, Boonman-de Winter LJ, Enneking T, Kuipers H, et al. Brisk walking compared with an individualised medical fitness programme for patients with type 2 diabetes: a randomised controlled trial. Diabetologia. 2008 May;5 I (5):736-46.

I 17. Van Sluijs EM, Van Poppel MN, Twisk JW, Brug J, Van Mechelen W. The positive effect on determinants of physical activity of a tailored, general practice-based physical activity intervention. Health Educ Res. 2005 Jun;20(3):345-56.

I 18. Wing RR. Long-term effects of a lifestyle intervention on weight and cardiovascular risk factors in individuals with type 2 diabetes mellitus: four-year results of the Look AHEAD trial. Arch Intern Med. 2010 Sep 27;170(I7):1566-75.

I 19. Praet SF, van Loon LJ. Exercise therapy in type 2 diabetes. Acta diabetologica. 2009 Dec;46(4):263-78.

120. Miller WR, Rollnick S. Motivational Interviewing: Preparing people to change addictive behaviour. New York: Guilford Press; 199I.

12I. Helmink JH, Kremers SP, van Boekel LC, van Brussel-Visser FN, de Vries NK. Factors determining the motivation of primary health care professionals to implement and continue the 'Beweegkuur' lifestyle intervention programme. J Eval Clin Pract. 2012 Jun; I8(3):682-8.

122. Helmink JH, Kremers SP, Van Boekel LC, Van Brussel-Visser FN, Preller L, De Vries NK. The BeweegKuur programme: a qualitative study of promoting and impeding factors for successful implementation of a primary health care lifestyle intervention for overweight and obese people. Fam Pract. 2012 Apr;29 Suppl I:i68-i74.

123. Gezondheidsraad. Richtlijnen goede voeding 2006 [Guidelines appropriate diet]. Den Haag: Gezondheidsraad. 2006.

124. Kwaliteitsinstituut voor de Gezondheidszorg CBO. Richtlijn: Diagnostiek en behandeling van obesitas bij volwassenen en kinderen [Guidelines: Diagnosis and treatment of obesity in adults and children]. Alphen aan den Rijn. 2008.

125. Nederlandse Diabetes Federatie. NDF Voedingsrichtlijn voor diabetes [NDF Food guidelines for diabetes]. 2006.

126. Craig CL, Marshall AL, Sjostrom M, Bauman AE, Booth ML, Ainsworth BE, et al. International physical activity questionnaire: 12 -country reliability and validity. Med Sci Sports Exerc. 2003 Aug;35(8): I 38 I -95. 
127. van Assema P, Brug J, Ronda G, Steenhuis I. The relative validity of a short Dutch questionnaire as a means to categorize adults and adolescents to total and saturated fat intake. J Hum Nutr Diet. 200 I Oct; I 4(5):377-90.

128. van Sloten TT, Savelberg HHCM, Duimel-Peeters IGP, Meijer K, Henry RMA, Stehouwer CDA, et al. Peripheral neuropathy, decreased muscle strength and obesity are strongly associated with walking in persons with type 2 diabetes without manifest mobility limitations. Diabetes research and clinical practice. 20II Jan;9I (I):32-9.

129. Valk GD, Nauta JJ, Strijers RL, Bertelsmann FW. Clinical examination versus neurophysiological examination in the diagnosis of diabetic polyneuropathy. Diabetic medicine : a journal of the British Diabetic Association. 1992 Oct;9(8):716-2I.

130. Gale CR, Martyn CN, Cooper C, Sayer AA. Grip strength, body composition, and mortality. International journal of epidemiology. 2007 Feb;36(I):228-35.

I3I. Sayer AA, Syddall HE, Dennison EM, Martin HJ, Phillips DI, Cooper C, et al. Grip strength and the metabolic syndrome: findings from the Hertfordshire Cohort Study. QJM. 2007 Nov; I00(I I):707-I3.

132. Podsiadlo D, Richardson S. The timed "Up \& Go": a test of basic functional mobility for frail elderly persons. J Am Geriatr Soc. 199| Feb;39(2): |42-8.

133. Astrand PO, Ryhming I. A nomogram for calculation of aerobic capacity (physical fitness) from pulse rate during sub-maximal work. J Appl Physiol. 1954 Sep;7(2):2 I821.

134. Cink RE, Thomas TR. Validity of the Astrand-Ryhming nomogram for predicting maximal oxygen intake. Br J Sports Med. 198। Sep;I5(3): I82-5.

135. Astrand I. Aerobic work capacity in men and women with special reference to age. Acta Physiol Scand Suppl. 1960;49(169): I -92.

136. Borg GA. Psychophysical bases of perceived exertion. Med Sci Sports Exerc. 1982;|4:377-8|.

137. Brooks R. EuroQol: the current state of play. Health Policy. 1996 Jul;37(I):53-72.

138. Dolan P. Modeling valuations for EuroQol health states. Med Care. 1997 Nov;35(I I): 1095-108.

139. Lamers LM, Stalmeier PFM, McDonnell J, Krabbe PFM, van Busschbach JJ. Kwaliteit van leven meten in economische evaluaties: het Nederlands EQ-5D-tarief. Ned Tijdschr Geneeskd. 2005; I49:I 574-8.

140. Oostenbrink JB, Koopmanschap MA, Rutten FF. Standardisation of costs: the Dutch Manual for Costing in economic evaluations. Pharmacoeconomics. 2002;20(7):443-54.

14I. Hakkaart-van Roijen L, Tan SS, Bouwmans CAM. Handleiding voor kostenonderzoek, methoden en standaard kostprijzen voor economische evaluaties in de gezondheidszorg. College voor Zorgverzekeringen, 2010.

142. Saunders RP, Evans MH, Joshi P. Developing a process-evaluation plan for assessing health promotion program implementation: a how-to guide. Health Promot Pract. 2005 Apr;6(2): 134-47.

143. Sallis JF, Bowles HR, Bauman A, Ainsworth BE, Bull FC, Craig CL, et al. Neighborhood Environments and Physical Activity Among Adults in II Countries. American Journal of Preventive Medicine. 2009;36(6):484-90.

144. Ekelund U, Griffin SJ, Wareham NJ. Physical activity and metabolic risk in individuals with a family history of type 2 diabetes. Diabetes Care. 2007 Feb;30(2):33 7-42. 
145. Laddu D, Dow C, Hingle M, Thomson C, Going S. A review of evidence-based strategies to treat obesity in adults. Nutrition in clinical practice : official publication of the American Society for Parenteral and Enteral Nutrition. 20 I I Oct;26(5):5 I 2-25.

146. Miller CT, Fraser SF, Levinger I, Straznicky NE, Dixon JB, Reynolds J, et al. The effects of exercise training in addition to energy restriction on functional capacities and body composition in obese adults during weight loss: a systematic review. PloS one. 2013;8(II):e81692.

147. Grant A, Treweek S, Dreischulte T, Foy R, Guthrie B. Process evaluations for cluster-randomised trials of complex interventions: a proposed framework for design and reporting. Trials. 20I3; I4:I5.

148. Oakley A, Strange V, Bonell C, Allen E, Stephenson J. Process evaluation in randomised controlled trials of complex interventions. BMJ. 2006 Feb I8;332(7538):4| 3-6.

149. Fleuren M, Wiefferink K, Paulussen T. Determinants of innovation within health care organizations: literature review and Delphi study. Int J Qual Health Care. 2004 Apr; 16(2):107-23.

150. Glasgow RE, Vogt TM, Boles SM. Evaluating the public health impact of health promotion interventions: the RE-AIM framework. Am J Public Health. 1999 Sep;89(9): I 322-7.

I5I. Steckler A, Linnan L. Process evaluation for public health interventions and research. San Francisco: John Wiley \& Sons, Inc.; 2002.

152. van der Meer FM, Couwenbergh BTLE, Enzing J], Ligtenberg G, Staal PC. De gecombineerde leefstijlinterventie: kosten, opbrengsten en de praktijk [The combined lifestyle intervention: costs, outcomes and practice]. College voor zorgverzekeringen (CVZ). 2010.

153. Schippers El. Herziening Zorgstelsel: Brief van de minister van volksgezondheid, welzijn en sport [Revision of Dutch health care: Letter of the minister of Health, Welfare and Sports]. Kamerstukken. 2010-201 I;29 689(nr. 326).

154. Mytton OT, Nnoaham K, Eyles H, Scarborough P, Ni Mhurchu C. Systematic review and meta-analysis of the effect of increased vegetable and fruit consumption on body weight and energy intake. BMC public health. 20I4; I4:886.

155. Swift DL, Johannsen NM, Lavie CJ, Earnest CP, Church TS. The role of exercise and physical activity in weight loss and maintenance. Progress in cardiovascular diseases. 20 I 4 Jan-Feb;56(4):44I-7.

I56. van Eijk-Hustings YJ, Daemen L, Schaper NC, Vrijhoef HJ. Implementation of Motivational Interviewing in a diabetes care management initiative in The Netherlands. Patient education and counseling. 20I I Jul;84(I ): I 0-5.

I57. van Dillen SM, Noordman J, van Dulmen S, Hiddink GJ. Quality of weight-loss counseling by Dutch practice nurses in primary care: an observational study. European journal of clinical nutrition. 2014 Jul 2.

158. Griffin SJ, Simmons RK, Prevost AT, Williams KM, Hardeman W, Sutton S, et al. Multiple behaviour change intervention and outcomes in recently diagnosed type 2 diabetes: the ADDITION-Plus randomised controlled trial. Diabetologia. 2014 Jul;57(7):I 308-I 9.

159. Barte JCM, Hendriks MRC, Rutten G, Veenhof C, Bemelmans WJE. Implementation of the 'BeweegKuur' in practice: utilization of care of a lifestyle intervention in the Netherlands. International Journal of Health Promotion and Education. 2014 20|4/07/04;52(4):222-8. 
160. Rogers EM. Diffusion of Innovations. New York: Free Press; 2003.

16I. Fayers PM, Jordhoy MS, Kaasa S. Cluster-randomized trials. Palliative medicine. 2002 Jan;I 6(I):69-70.

162. Farrin A, Russell I, Torgerson D, Underwood M, Team UBT. Differential recruitment in a cluster randomized trial in primary care: the experience of the UK back pain, exercise, active management and manipulation (UK BEAM) feasibility study. Clinical trials. 2005;2(2): 1 19-24.

163. Helmink JHM, Raaijmakers LGM, Rutten GM, De Vries NK, Kremers SPJ. Gecombineerde leefstijl interventies in Nederland: ervaringen uit de BeweegKuur [Combined lifestyle interventions in the Netherlands: experiences gained with the BeweegKuur intervention]. Tijdschrift voor Gezondheidswetenschappen. 20I3;20I3(2):88-90.

164. Green J, Tones K. Health Promotion. 2nd ed. London: SAGE Publications Ltd; 2010.

165. Diabetes Prevention Program Research Group, Knowler WC, Fowler SE, Hamman RF, Christophi CA, Hoffman HJ, et al. I0-year follow-up of diabetes incidence and weight loss in the Diabetes Prevention Program Outcomes Study. Lancet. 2009 Nov 14;374(9702): 1677-86.

166. Diabetes Prevention Program Research G. The Diabetes Prevention Program (DPP): description of lifestyle intervention. Diabetes Care. 2002 Dec;25(I 2):2 I 65-7I .

167. Wadden TA, Neiberg RH, Wing RR, Clark JM, Delahanty LM, Hill JO, et al. Four-year weight losses in the Look AHEAD study: factors associated with long-term success. Obesity (Silver Spring). 20 I I Oct; I9(10): I 987-98.

168. Berendsen BAJ, Kremers SPJ, Savelberg HHCM, Schaper NC, Hendriks MRC. The implementation and sustainability of a combined lifestyle intervention in primary care: mixed method process evaluation. BMC family practice. 20I5;16:37.

169. van Poppel MN, Chinapaw MJ, Mokkink LB, van Mechelen W, Terwee CB. Physical activity questionnaires for adults: a systematic review of measurement properties. Sports medicine. 2010 Jul I;40(7):565-600.

170. IPAQ Group. Guidelines for data processing and analysis of the International Physical Activity Questionnaire (IPAQ) 2005. Available from: www.ipaq.ki.se.

17I. van Assema P, Brug J, Ronda G, Steenhuis I, Oenema A. A short dutch questionnaire to measure fruit and vegetable intake: relative validity among adults and adolescents. Nutrition and health. 2002;16(2):85-106.

172. Lindstrom J, Peltonen M, Eriksson JG, Aunola S, Hamalainen H, llanne-Parikka P, et al. Determinants for the effectiveness of lifestyle intervention in the Finnish Diabetes Prevention Study. Diabetes Care. 2008 May;3 I (5):857-62.

173. Azar KM, Xiao L, Ma J. Baseline obesity status modifies effectiveness of adapted diabetes prevention program lifestyle interventions for weight management in primary care. BioMed research international. 2013;20I3:191209.

174. O'Connell SE, Griffiths PL, Clemes SA. Seasonal variation in physical activity, sedentary behaviour and sleep in a sample of UK adults. Annals of human biology. 20 I 4 Jan-Feb;4I (I): I-8.

175. Tucker P, Gilliland J. The effect of season and weather on physical activity: a systematic review. Public health. 2007 Dec; I 2 I (I 2):909-22.

176. Twisk J, de Vente W. Attrition in longitudinal studies. How to deal with missing data. Journal of clinical epidemiology. 2002 Apr;55(4):329-37.

177. King AC, Castro CM, Buman MP, Hekler EB, Urizar GG, Jr., Ahn DK. Behavioral impacts of sequentially versus simultaneously delivered dietary plus physical activity 
interventions: the CALM trial. Annals of behavioral medicine : a publication of the Society of Behavioral Medicine. 2013 Oct;46(2):157-68.

178. Twisk J, de Boer M, de Vente W, Heymans M. Multiple imputation of missing values was not necessary before performing a longitudinal mixed-model analysis. Journal of clinical epidemiology. 2013 Sep;66(9): I022-8.

179. Helmink JHM, Boekel LC, Kremers SPJ. Implementatie van de BeweegKuur: Monitorstudie onder zorgverleners [Implementation of the BeweegKuur: Monitoring study in health care providers]. Maastricht University. $20 \mathrm{II}$.

180. Helmink JH, Gubbels JS, van Brussel-Visser FN, de Vries NK, Kremers SP. Baseline predictors of maintenance of intervention-induced changes in physical activity and sitting time among diabetic and pre-diabetic patients: a descriptive case series. BMC research notes. 2013;6:190.

I8I. Kozey-Keadle S, Staudenmayer J, Libertine A, Mavilia M, Lyden K, Braun B, et al. Changes in sedentary time and physical activity in response to an exercise training and/or lifestyle intervention. J Phys Act Health. 2014 Sep; I I (7):I 324-33.

182. Lee PH, Macfarlane DJ, Lam TH, Stewart SM. Validity of the International Physical Activity Questionnaire Short Form (IPAQ-SF): a systematic review. Int J Behav Nutr Phys Act. 201 I;8:II 5.

183. Sakurai Y, Teruya K, Shimada N, Umeda T, Tanaka H, Muto T, et al. Association between duration of obesity and risk of non-insulin-dependent diabetes mellitus The Sotetsu Study. Am J Epidemiol. 1999 Feb I;| 49(3):256-60.

184. Sparling PB, Howard BJ, Dunstan DW, Owen N. Recommendations for physical activity in older adults. BMJ. 20I5;350:h I00.

185. Welten DC, Heymans J, van der Meer FM. Standpunt Bariatrische Chirurgie [Position Statement Bariatric Surgery]. Zorginstituut Nederland. 2014.

186. Katzmarzyk PT, Church TS, Craig CL, Bouchard C. Sitting Time and Mortality from All Causes, Cardiovascular Disease, and Cancer. Med Sci Sport Exer. 2009 May;4 I (5):998-I 005.

187. Hamilton MT, Healy GN, Dunstan DW, Zderic TW, Owen N. Too Little Exercise and Too Much Sitting: Inactivity Physiology and the Need for New Recommendations on Sedentary Behavior. Current cardiovascular risk reports. 2008 Jul;2(4):292-8.

188. Gibbs BB, Brancati FL, Chen H, Coday M, Jakicic JM, Lewis CE, et al. Effect of improved fitness beyond weight loss on cardiovascular risk factors in individuals with type 2 diabetes in the Look AHEAD study. European journal of preventive cardiology. 20 I 4 May;2 I (5):608-I 7.

189. Kirk-Sanchez NJ, McGough EL. Physical exercise and cognitive performance in the elderly: current perspectives. Clin Interv Aging. 20 I4;9.

190. Padilla C, Perez L, Andres P. Chronic exercise keeps working memory and inhibitory capacities fit. Front Behav Neurosci. 2014 Mar II;8.

19I. Van Dijk ML, De Groot RHM, Savelberg HHCM, Van Acker F, Kirschner PA. The Association Between Objectively Measured Physical Activity and Academic Achievement in Dutch Adolescents: Findings From the GOALS Study. J Sport Exercise Psy. 20I4 Oct;36(5):460-73.

192. Williamson DA, Rejeski J, Lang W, Van Dorsten B, Fabricatore AN, Toledo K, et al. Impact of a weight management program on health-related quality of life in overweight adults with type 2 diabetes. Arch Intern Med. 2009 Jan 26;169(2):I63 -7I.

193. Dunkley AJ, Bodicoat DH, Greaves CJ, Russell C, Yates T, Davies MJ, et al. Diabetes prevention in the real world: effectiveness of pragmatic lifestyle interventions for the 
prevention of type 2 diabetes and of the impact of adherence to guideline recommendations: a systematic review and meta-analysis. Diabetes Care. 2014 Apr;37(4):922-33.

194. Brocklebank LA, Falconer CL, Page AS, Perry R, Cooper AR. Accelerometermeasured sedentary time and cardiometabolic biomarkers: A systematic review. Prev Med. 20 I 5 Jul;76:92-I02.

195. Australian Government Department of Health. Australia's Physical Activity and Sedentary Behaviour Guidelines. 2014.

196. Davies S, Jewell T, Burns H, McBride M. Start Active, Stay Active - A report on physical activity for health from the four home countries' Chief Medical Officers UK Physical Activity Guidelines. 20I I.

197. Helmink JHM, Boekel LC, van der Sluis ME, Kremers SPJ. Lange termijn evaluatie onder deelnemers aan de BeweegKuur: Rapportage van de resultaten van een followup meting bij deelnemers [Long term evaluation in BeweegKuur participants: Report of results of a follow-up measurement in participants]. Maastricht University. $201 \mathrm{I}$.

198. Ainsworth BE, Haskell WL, Herrmann SD, Meckes N, Bassett DR, Jr., Tudor-Locke C, et al. 201 I Compendium of Physical Activities: a second update of codes and MET values. Med Sci Sports Exerc. 20 I I Aug;43(8):I575-8 I.

199. Hu FB, Li TY, Colditz GA, Willett WC, Manson JE. Television watching and other sedentary behaviors in relation to risk of obesity and type 2 diabetes mellitus in women. Jama. 2003 Apr 9;289(I4): I785-9I.

200. Laaksonen DE, Lindstrom J, Lakka TA, Eriksson JG, Niskanen L, Wikstrom K, et al. Physical activity in the prevention of type 2 diabetes: the Finnish diabetes prevention study. Diabetes. 2005 Jan;54(I): 158-65.

20I. Lange RM, Nies MA. Benefits of walking for obese women in the prevention of bone and joint disorders. Orthopedic nursing. 2004 May-Jun;23(3):2 I I-5.

202. Villareal DT, Banks M, Sinacore DR, Siener C, Klein S. Effect of weight loss and exercise on frailty in obese older adults. Arch Intern Med. 2006 Apr 24;166(8):860-6.

203. Meijer EP, Westerterp KR, Verstappen FT. Effect of exercise training on total daily physical activity in elderly humans. European journal of applied physiology and occupational physiology. 1999 Jun;80(I):16-2I.

204. Westerterp KR. Daily physical activity and ageing. Curr Opin Clin Nutr Metab Care. 2000 Nov;3(6):485-8.

205. Martin A, Fitzsimons C, Jepson R, Saunders DH, van der Ploeg HP, Teixeira PJ, et al. Interventions with potential to reduce sedentary time in adults: systematic review and meta-analysis. Br J Sports Med. 20I5 Aug;49(I6):I056-63.

206. Kaiser K.A., Brown A.W., Bohan Brown M.M., Shikany J.M., Mattes R.D., D.B. A. Increased fruit and vegetable intake has no discernible effect on weight loss: a systematic review and meta-analysis. Am J Clin Nutr. 20I4;Aug; I00(2):567-76.

207. Ball L, Leveritt M, Cass S, Chaboyer W. Effect of nutrition care provided by primary health professionals on adults' dietary behaviours: a systematic review. Fam Pract. 2015 Aug 19.

208. Bray GA, Bouchard C. Handbook of Obesity: Clinical Applications. 2nd ed. New York: Marcel Dekker; 2004.

209. Langenberg C, Sharp SJ, Schulze MB, Rolandsson O, Overvad K, Forouhi NG, et al. Long-term risk of incident type 2 diabetes and measures of overall and regional obesity: the EPIC-InterAct case-cohort study. PLoS medicine. 20I2;9(6):el 001230. 
210. Ekelund U, Ward HA, Norat T, Luan J, May AM, Weiderpass E, et al. Physical activity and all-cause mortality across levels of overall and abdominal adiposity in European men and women: the European Prospective Investigation into Cancer and Nutrition Study (EPIC). The American journal of clinical nutrition. 2015 Mar; I I I (3):6 I 3-2I.

21 I. Christian JG, Bessesen DH, Byers TE, Christian KK, Goldstein MG, Bock BC. Clinic based support to help overweight patients with type 2 diabetes increase physical activity and lose weight. Arch Intern Med. 2008 Jan 28; I68(2): I 4 I -6.

212. Leblanc ES, O'Connor E, Whitlock EP, Patnode CD, Kapka T. Effectiveness of primary care-relevant treatments for obesity in adults: a systematic evidence review for the U.S. Preventive Services Task Force. Annals of internal medicine. $201 \mathrm{I}$ Oct 4;155(7):434-47.

213. Sargent GM, Forrest LE, Parker RM. Nurse delivered lifestyle interventions in primary health care to treat chronic disease risk factors associated with obesity: a systematic review. Obes Rev. 2012 Dec; |3(I2): I |48-7|.

2I4. Johns DJ, Hartmann-Boyce J, Jebb SA, Aveyard P, Behavioural Weight Management Review G. Diet or exercise interventions vs combined behavioral weight management programs: a systematic review and meta-analysis of direct comparisons. Journal of the Academy of Nutrition and Dietetics. 2014 Oct; I I 4(I0): I557-68.

2 I5. Franz MJ, VanWormer JJ, Crain AL, Boucher JL, Histon T, Caplan W, et al. Weightloss outcomes: a systematic review and meta-analysis of weight-loss clinical trials with a minimum I-year follow-up. Journal of the American Dietetic Association. 2007 Oct; I 07(I0): I 755-67.

216. Oman RF, King AC. The effect of life events and exercise program format on the adoption and maintenance of exercise behavior. Health psychology : official journal of the Division of Health Psychology, American Psychological Association. 2000 Nov; 19(6):605-I2.

217. Helmink JH, Cox VCM, Kremers SPJ. Implementatie van de BeweegKuur: Een pilot studie [Implementation of the BeweegKuur: A pilot study]. Maastricht University. 2009.

2 18. Helmink JHM, Meis JJM, Kremers SPJ. Een jaar BeweegKuur, en dan? Een onderzoek naar bevorderende en belemmerende contextuele factoren [One year of BeweegKuur, now what? A study of promoting and impeding contextual factors]. Maastricht University. 2010.

219. Rappange DR, Brouwer WBF. The evaluation of lifestyle interventions in the Netherlands. Health Econ Policy L. 2012 Apr;7(2):243-6I.

220. Barte JC, Wendel-Vos GC. A Systematic Review of Financial Incentives for Physical Activity: The Effects on Physical Activity and Related Outcomes. Behavioral medicine. 20I5 Oct 2:0.

22I. van der Weegen S, Verwey R, Spreeuwenberg M, Tange H, van der Weijden T, de Witte L. It's LiFe! Mobile and Web-Based Monitoring and Feedback Tool Embedded in Primary Care Increases Physical Activity: A Cluster Randomized Controlled Trial. Journal of medical Internet research. 2015;17(7):el84.

222. Kerry SM, Bland JM. The intracluster correlation coefficient in cluster randomisation. BMJ. I 998 May 9;3 I6(7|42): I455.

223. Kerry SM, Bland JM. Sample size in cluster randomisation. BMJ. 1998 Feb I 4;3 I 6(7| 30):549. 
224. Eldridge SM, Ashby D, Kerry S. Sample size for cluster randomized trials: effect of coefficient of variation of cluster size and analysis method. International journal of epidemiology. 2006 Oct;35(5): I 292-300.

225. Rutterford C, Copas A, Eldridge S. Methods for sample size determination in cluster randomized trials. International journal of epidemiology. 20 I 5 Jun;44(3): I 05 I -67.

226. Tuomilehto H, Gylling H, Peltonen M, Martikainen T, Sahlman J, Kokkarinen J, et al. Sustained improvement in mild obstructive sleep apnea after a diet- and physical activity-based lifestyle intervention: postinterventional follow-up. The American journal of clinical nutrition. 2010 Oct;92(4):688-96.

227. Jacobson TA. Opening a New Lipid "Apo-thecary": Incorporating Apolipoproteins as Potential Risk Factors and Treatment Targets to Reduce Cardiovascular Risk. Mayo Clin Proc. 20I I Aug;86(8):762-80.

228. van der Weegen S, Verwey R, Spreeuwenberg M, Tange $H$, van der Weijden $T$, de Witte $\mathrm{L}$. The development of a mobile monitoring and feedback tool to stimulate physical activity of people with a chronic disease in primary care: a user-centered design. JMIR mHealth and uHealth. 20I3; I (2):e8.

229. Bort-Roig J, Gilson ND, Puig-Ribera A, Contreras RS, Trost SG. Measuring and influencing physical activity with smartphone technology: a systematic review. Sports medicine. 20 I 4 May;44(5):67 I -86.

230. Middelweerd A, Mollee JS, van der Wal CN, Brug J, Te Velde SJ. Apps to promote physical activity among adults: a review and content analysis. Int J Behav Nutr Phys Act. 2014;1 I:97.

23I. Belanger-Gravel A, Godin G, Vezina-Im LA, Amireault S, Poirier P. The effect of theory-based interventions on physical activity participation among overweight/obese individuals: a systematic review. Obes Rev. 20 I I Jun; I2(6):430-9.

232. Loket Gezond Leven. BeweegKuur: Eerste aanwijzingen voor effectiviteit https://www.loketgezondleven.nl/interventies/i-database/I402397: RIVM Centrum Gezond Leven; [March II, 20I6]. 
Summary 
The numbers of people who are overweight, obese and/or physically inactive are rising worldwide, leading to increased risk for non-communicable diseases, such as type 2 diabetes and cardiovascular disease, and high health care costs. The two main behaviours responsible for excess weight are physical inactivity and unhealthy diet. Combined lifestyle interventions can target these two behaviours simultaneously, and thereby reduce the direct risk of physical inactivity and diet on cardio-metabolic risk profile, but also reduce the risk for metabolic diseases through weight loss. Long-term behavioural change can be achieved by applying the existing theoretical framework of the self-determination theory and by using behavioural change techniques such as Motivational Interviewing. The majority of studies aimed at combined lifestyle interventions are set in a controlled clinical research setting, limiting translation of results to the real world. In addition, it is unclear whether group cohesion and the introduction to exercise under supervision of a physical activity specialist yields more effects than guidance solely based on Motivational Interviewing. Because the primary care has been suggested to be the optimal setting for identification and delivery of lifestyle interventions for overweight and obesity, there is a need for well-designed studies in this setting.

Accurate assessment of physical activity is needed to provide insight in the effectiveness of physical activity interventions. Nonetheless, the optimal way to estimate physical activity level remains ambiguous. Subjective methods like questionnaires suffer from recall bias and social desirability, whereas they are easy to use in large-scale studies. An innovative method like accelerometry can estimate physical activity behaviour and does not suffer from subjectivity, but quality of the estimates should be assessed carefully before applying the devices in scientific research.

The aims of this thesis were to evaluate I) the quality of estimates of physical activity behaviour by accelerometers, and 2) the effectiveness of additional guidance in the BeweegKuur intervention executed in primary care in the Netherlands.

Chapter 2 revealed the importance of selecting the right activity monitor for the assessment of physical activity and sedentary behaviour. The reliability, validity and user friendliness of three commercially available tri-axial activity monitors was studied in both a laboratory and real-life setting. The two monitors that were worn on the upper thigh, the ActivPAL3 and CAM, provided valid estimates of sitting and standing time in the lab. The hip-worn monitor, the ActiGraph GT3X, was not able to accurately discriminate between sitting and standing, limiting the applicability in studies aimed at sedentary behaviour. Active time was estimated correctly in all three monitors; however, reliability of the CAM during walking at a moderate to high intensity was questionable. Further, the ActiGraph 
GT3X showed highest user friendliness, followed by ActivPAL3. The quality of estimates and user friendliness varied between activity monitors, and it was concluded that it differs per study design and study goal which device can be used.

Chapter 3 showed which algorithm is optimal for the identification of non-wear in accelerometry data among participants with overweight. An algorithm is required to identify and remove periods of non-wear from accelerometry data and thereby improve the accuracy of estimates of physical activity and sitting time. Non-wear is recognised as a continuous bout of inactivity of a minimum length, i.e. the time window. Because overweight participants are often assumed to be more sedentary, their optimal time window was expected to be larger than the commonly used time windows of 60 and 90 minutes. Strikingly, the optimal time window in our study population with overweight was 20 minutes. Applying an algorithm on raw tri-axial data improved the sensitivity of the algorithm. This study emphasized the crucial role of performing well-chosen data treatment in activity monitoring by means of accelerometry.

Chapter 4 described the design of a randomised controlled trial aimed to study the differences in effect between two doses (i.e. the start-up programme versus the supervised programme) of a combined lifestyle intervention, the BeweegKuur. The BeweegKuur is a primary care intervention lasting one year aimed to improve lifestyle in people with overweight or obesity. Both BeweegKuur programmes consisted of six meetings with a lifestyle advisor (LSA), six or seven individual meetings with a physiotherapist and several individual and group meetings with a dietician. Additionally, the supervised programme also comprised 26-34 group meetings with a physiotherapist. Guidance within the BeweegKuur was based on the self-determination theory and applied the techniques of Motivational Interviewing and goal setting. The trial was designed as a clustered multi-centre RCT to prevent contamination between participants and health care providers. Thirty health care clusters, consisting of a LSA, physiotherapist and dietician, were randomized to either the start-up or the supervised programme. The time horizon was two years, including a one year follow-up after the end of the intervention period. The primary outcome (physical activity) and the secondary outcomes (sitting time and diet) were self-reported at baseline and each six months. Other objectively measured secondary outcomes (e.g. weight, blood pressure and fitness) were assessed yearly.

The process evaluation of the BeweegKuur study was described in chapter 5. Interviews with health care professionals and questionnaires from participants revealed that 
participants in both programmes were very satisfied with the delivered guidance. Adherence to the protocol varied between health care clusters, and the number of attended meetings was lower than prescribed in the protocol. Main reasons for not planning or attending all meetings were physical problems or illness, lack of motivation, unrealistic expectations towards the guidance or the effects and planning issues. Participants who attended more group meetings with the physiotherapist, reported to attend less meetings with the LSA and dietician, indicating that there might be a feasibility issue in the amount of guidance. Interviews with the health care professionals showed that the continuation of the BeweegKuur or similar interventions in primary care was challenging, mainly due to capacity and financial issues. Re-invention of the intervention was crucial for feasibility. Furthermore, sustainable funding for prevention programmes in primary care was required for long-term continuation of the intervention.

The effect evaluation described in chapter $\mathbf{6}$ argued that the additional guidance in the supervised programme did not seem to lead to beneficial effects regarding physical activity, diet and health, compared to the start-up programme. The addition of extra group meetings to overcome barriers towards physical activity did thus not yield the expected benefits in the current trial. Nevertheless, participants in both groups improved their lifestyle regarding walking time and dietary behaviour. Moreover, in both programmes weight and waist circumference significantly decreased at 12 and 24 months after the start of the intervention.

Chapter 7 discussed the results, implications and methodological considerations of the studies presented in this thesis by relating it to existing literature. The setting of the BeweegKuur in primary care enabled us to draw conclusions about the implementation and effectiveness of the programmes in real-life. Nonetheless, the programmes were not compared to a group without an intervention, limiting the ability to draw conclusions about the changes we observed in participants of both groups over time. Moreover, health care professionals did not recruit the required number of participants (which they confirmed to be feasible prior to study start), implying that the potential difference between the programmes remained undetected due to insufficient power. The contents of the programmes were altered by health care professionals to prevent the drop-out of participants and health care clusters. A process evaluation should be an integral part of effectiveness trials. Also, intervention aspects that are allowed to be altered and aspects should be executed as prescribed in the protocol should be determined prior to implementation. Our study presented self-reported physical activity behaviour, because 
the number of valid accelerometry measurements was inadequate for analyses and the reliability of the estimates at moderate to high intensity activity appeared to be insufficient. Future research should only apply valid, reliable and user friendly accelerometers and extensive planning is required to acquire accurate measurements in large longitudinal trials in real-life setting. Studies utilizing activity monitors should be transparent about data acquirement and data treatment, but even more important, consensus about the measurement of physical activity behaviour is necessary to enable proper comparison between studies.

In conclusion, this thesis revealed the crucial role of profound decision making when utilizing accelerometry for the estimation of physical behaviour. Regarding combined lifestyle interventions, it indicated a potential threshold for the amount of simultaneous dietary and physical activity guidance and it identified barriers and success factors for sustainability of combined lifestyle interventions in the primary care setting. 

Samenvatting 
Overgewicht en obesitas komen steeds vaker voor, en leiden gezamenlijk met verminderde fysieke activiteit tot een significant groter risico op chronische aandoeningen, zoals diabetes en hart- en vaatziekten. Deze chronische aandoeningen gaan gepaard met hoge gezondheidszorgkosten. Overgewicht wordt grotendeels veroorzaakt door ongezond eetgedrag en een gebrek aan fysieke activiteit, twee factoren die gelijktijdig aangepakt kunnen worden in een gecombineerde leefstijlinterventie. Door gezonder te eten en meer te bewegen verlaagt enerzijds het directe risico op chronische aandoeningen, en anderzijds daalt ook de mate van overgewicht en de negatieve invloed daarvan op de gezondheid.

Gecombineerde leefstijlinterventies richten zich op het verbeteren van meerdere gedragingen, zoals fysieke activiteit en eetgedrag, en kunnen worden toegepast door de eerstelijns gezondheidszorg (o.a. huisarts, fysiotherapeut en diëtist). Het aannemen en volhouden van een gezondere leefstijl wordt gestimuleerd als zorgverleners gebruik maken van de self-determination theory en gesprekstechnieken zoals motivational interviewing. Deze technieken zijn gericht op het bevorderen van de intrinsieke motivatie: motivatie vanuit de persoon zelf. Over het algemeen lijken gecombineerde leefstijlinterventies effectief; echter, de meeste wetenschappelijke studies hebben plaatsgevonden in een gecontroleerde onderzoeksomgeving. De bevindingen zijn daarom niet goed te generaliseren naar de dagelijkse praktijk. Onderzoek in de eerstelijnszorg is essentieel, omdat juist die zorgverleners (m.n. huisarts, praktijkverpleegkundige, fysiotherapeut en diëtist) een grote rol spelen in de identificatie en behandeling van overgewicht met behulp van gecombineerde leefstijlinterventies. Bovendien is het onbekend of het bewegen in een groep en onder begeleiding extra effect heeft naast één-op-één begeleiding in de vorm van motivational interviewing.

Om de effecten van leefstijlinterventies te meten, is een accurate meetmethode van fysieke activiteit noodzakelijk. Vragenlijsten worden hierbij vaak gebruikt vanwege de goede praktische toepasbaarheid, maar zijn vaak beperkt in validiteit. Versnellingsmeters in de vorm van activiteitenmonitors bieden een objectieve methode om fysieke activiteit te meten, maar de kwaliteit van de metingen vereist onderzoek.

Deze thesis beschrijft onderzoek naar I) de kwaliteit van metingen van fysieke activiteit en zittijd door versnellingsmeters, en 2) de effectiviteit van extra groepsbegeleiding in een gecombineerde leefstijlinterventie uitgevoerd in de eerstelijnszorg. 
Hoofdstuk 2 beschreef een studie naar de betrouwbaarheid, validiteit en gebruiksvriendelijkheid van drie versnellingsmeters in zowel een gecontroleerde laboratoriumsetting als in het dagelijks leven. De versnellingsmeters die op het bovenbeen gedragen werden, de ActivPAL3 en de CAM, leverden accurate schattingen van sta- en zittijd in het lab. De meter gedragen op de heup, de ActiGraphGT3X, kon geen correct onderscheid maken tussen zitten en staan, waardoor de toepasbaarheid in studies naar sedentair gedrag beperkt is. De drie versnellingsmeters waren in staat de tijd in beweging goed te schatten; echter, de meting van de CAM bij lopen met een matige tot hoge intensiteit leek onbetrouwbaar. De ActiGraphGT3X en de ActivPAL3 scoorden het hoogst qua gebruiksvriendelijkheid. Kortom, de kwaliteit en gebruiksvriendelijkheid van de versnellingsmeters varieerde, en welke meter het meest geschikt is, hangt af van het onderzoeksdesign en -doel.

Versnellingsmeters worden niet altijd continu door onderzoeksdeelnemers gedragen. Als de tijd waarin de meter niet gedragen is, niet uit de meting verwijderd wordt, kan de hoeveelheid zittijd sterk overschat worden. Het onderzoek in hoofdstuk 3 liet zien welk algoritme optimaal is voor het identificeren en verwijderen van meettijd waarin de versnellingsmeter niet gedragen is. Het algoritme herkent zogenaamde non-wear als zich een langdurige periode van inactiviteit voordoet. Deze periode heeft een minimale duur, om te voorkomen dat ieder zitmoment wordt geïdentificeerd als non-wear. Er werd verwacht dat de minimale duur van het algoritme langer zou zijn dan de gebruikelijke duur van 60 en 90 minuten, omdat wordt aangenomen dat mensen met overgewicht meer zitten. Echter, doordat het algoritme werd toegepast op de ruwe versnellingsdata, bleek een duur van 20 minuten het meest optimaal te zijn voor het identificeren van non-wear bij mensen met overgewicht.

Hoofdstuk 4 beschreef de opzet van een gerandomiseerd, gecontroleerd onderzoek gericht op het verschil in effecten tussen twee versies van de BeweegKuur, een gecombineerde leefstijlinterventie in de eerstelijnszorg. Gedurende één jaar werden mensen met overgewicht of obesitas begeleid naar een gezondere leefstijl. Beide programma's bevatten zes persoonlijke gesprekken met de leefstijladviseur (LSA), zes of zeven individuele sessies met de fysiotherapeut en een aantal individuele en groepssessies 
bij de diëtist. In het intensievere programma, het 'begeleid bewegen', ontvingen de deelnemers extra 26 tot 34 groepssessies onder begeleiding van de fysiotherapeut. De begeleiding was gebaseerd op de self-determination theory en bevatte motivational interviewing en goal setting. Dertig eerstelijns gezondheidszorglocaties werden gerandomiseerd in de controle conditie ('opstart') of de experimentele conditie ('begeleid bewegen') om contaminatie tussen deelnemers en zorgverleners te voorkomen. Fysieke activiteit, eetgedrag en gezondheidsparameters van de deelnemers werden gemeten gedurende de één-jarige interventie, en het behoud werd gedurende het tweede jaar gemeten. Fysieke activiteit en eetgedrag werden ieder half jaar gerapporteerd aan de hand van vragenlijsten. Gezondheidsparameters (o.a. BMI, bloeddruk en fitheid) werden jaarlijks gemeten door de zorgverleners.

In hoofdstuk 5 werden de bevindingen van de procesevaluatie van het BeweegKuuronderzoek beschreven, gebaseerd op interviews met zorgverleners en vragenlijsten afgenomen bij de deelnemers. Deelnemers van beide programma's bleken tevreden met de begeleiding die zij ontvangen hadden. De uitvoering van de programma's varieerde echter tussen de zorglocaties, waarbij er over het algemeen minder begeleiding had plaatsgevonden dan in het protocol, met name vanwege lichamelijke klachten, gebrek aan motivatie, te hoge verwachtingen en problemen met de planning. Meer begeleiding door de fysiotherapeut ging gepaard met minder begeleiding door LSA en diëtist. Verder was de voortzetting van de BeweegKuur een uitdaging, voornamelijk vanwege problemen qua capaciteit en financiering. Om haalbaarheid in de praktijk te waarborgen, moeten zorgverleners toegestaan worden om onderdelen van de interventie aan te passen (zogenaamde re-invention). Daarnaast is blijvende financiering noodzakelijk voor het duurzame aanbod van gecombineerde leefstijlinterventies in de eerstelijnszorg.

De evaluatie van de effecten in hoofdstuk 6 suggereerde dat de extra groepsbegeleiding in het programma met 'begeleid bewegen' niet heeft geleid tot extra effecten op fysieke activiteit, eetgedrag en gezondheid, ten opzichte van het 'opstart' programma. De verwachte effecten van de groepsvorming, de langdurige ervaring met beweging en begeleiding door de fysiotherapeut werden niet gevonden. Desondanks wandelden deelnemers van beide programma's meer en verbeterde eetgedrag na een jaar deelname, 
en dat bleef behouden na een jaar zonder begeleiding. Daarbij daalde gewicht en buikomvang van beide groepen deelnemers significant één en twee jaar na de start.

In hoofdstuk 7 volgde een discussie van de resultaten, implicaties en methodologische overwegingen van de studies in deze thesis. Omdat het onderzoek naar de twee programma's van de BeweegKuur daadwerkelijk in de eerstelijnszorg plaatsvond, kunnen de conclusies over de implementatie en effecten gegeneraliseerd worden naar de dagelijkse praktijk. Echter, deelnemers aan de programma's werden niet vergeleken met een groep zonder interventie, waardoor niet met zekerheid geconcludeerd kon worden dat de verbeteringen in beide groepen veroorzaakt waren door deelname aan de BeweegKuur. Bovendien was de benodigde doelgroepgrootte niet bereikt, waardoor het verschil tussen de effecten van de programma's wellicht niet aan het licht was gekomen. Zorgverleners pasten de begeleiding in de programma's aan om uitval van de praktijken en deelnemers te voorkomen. De procesevaluatie leverde essentiële informatie over de implementatie en de duurzaamheid van de BeweegKuur, en zou daarom geïntegreerd moeten worden in toekomstige en lopende effectevaluaties. Bovendien moet voorafgaand aan de implementatie van gecombineerde leefstijlinterventies bepaald worden welke onderdelen van de interventie wel en welke onderdelen niet aangepast mogen worden om de haalbaarheid en de effectiviteit in de praktijk te waarborgen. De evaluatie van de BeweegKuur was gebaseerd op zelf gerapporteerde fysieke activiteit, omdat het aantal deelnemers met valide metingen van de versnellingsmeter te laag was en de betrouwbaarheid van de versnellingsmeter niet voldoende aangetoond was. Toekomstig onderzoek moet gebruik maken van valide, betrouwbare en gebruiksvriendelijke versnellingsmeters. Verder is gedetailleerde planning nodig om accurate metingen met versnellingsmeters te verkrijgen in grote longitudinale studies in de praktijk. Daarnaast zou onderzoek met versnellingsmeters duidelijk moeten aangeven op welke wijze de data verzameld is en op welke manier de dataverwerking heeft plaatsgevonden om studieresultaten te kunnen vergelijken.

Deze thesis heeft aangetoond dat een doordachte keuze voor een versnellingsmeter en de verwerking van de versnellingssignalen cruciaal is voor correcte metingen van fysieke activiteit en zittijd. Verder suggereerde de data dat de effectiviteit en haalbaarheid van de hoeveelheid begeleiding binnen gecombineerde leefstijlinterventies een bovengrens heeft, 
en dat verschillende barrières en succesfactoren een rol spelen in de voortzetting van gecombineerde leefstijlinterventies in de eerstelijnszorg in Nederland. 
Dankwoord 
In dit hoofdstuk bedank ik graag iedereen die mij heeft geholpen om mijn promotie voor elkaar te krijgen. Voor de samenwerking, inbreng van kennis, steun gedurende de moeilijkere tijden in onderzoek, maar natuurlijk ook voor de gezelligheid en het plezier tijdens deze leuke periode.

Allereerst wil ik mijn promotors en copromotor bedanken voor de begeleiding van mijn promotietraject. Hans, je hebt mij de kans en de vrijheid gegeven om mijn eigen draai te geven aan het project, met als resultaat twee niet-geplande, maar zeker zo leuke artikelen in dit boekje en de ontwikkeling van mijn expertise op het gebied van activiteitenmonitoring. Verder heeft jouw realistische houding mij tijdens de uitdagingen van het BeweegKuuronderzoek talloze keren gerustgesteld. Geheel terecht ben je nog op tijd gepromoveerd tot mijn promotor. Marike, we hebben de afgelopen jaren nauw samengewerkt om het onderzoek rond de BeweegKuur tot een goed einde te brengen. Wat een kilometers hebben we voor dit project afgelegd; we hebben samen heel Nederland doorgecrosst om de praktijken te bezoeken. We kwamen onderweg grote en kleine hindernissen tegen, maar wisten altijd samen tot een oplossing te komen waarmee ons werk door kon gaan en de kwaliteit van het onderzoek gewaarborgd werd. Nicolaas, vooral het laatste jaar hebben wij veel gediscussieerd over de analyse en resultaten. We kwamen hierdoor tot veel inzichten over de vele leerpunten van het onderzoek. Van je kritische, en ook klinische blik op het onderzoek heb ik veel geleerd en dat heeft het proefschrift zeker goed gedaan.

De beoordelingscommissie, Prof. Dr. Nanne de Vries, Prof. Dr. Marleen van Baak, Prof. Dr. Greet Cardon, Prof. Dr. Ab Klink en Prof. Dr. Trudy van der Weijden wil ik bedanken voor het lezen en beoordelen van dit proefschrift. De corona bedank ik voor hun aanwezigheid en het lezen van mijn proefschrift.

Toen ik in 2010 aan het BeweegKuuronderzoek begon, werd ik verwelkomd door een geweldig gemotiveerd en gezellig team. BeweegKuurcollega's Judith en Leonieke, jullie zijn met ons meegegaan op de eerste praktijkbezoeken en hebben ons wegwijs gemaakt in de wereld van de Beweegkuur. Het was een mooi begin van het project, bedankt hiervoor! lk heb veel mogen samenwerken met Stef, Geert en Jessie. Jullie input heeft geresulteerd in een brede visie op de BeweegKuur als interventie en de daarbij horende processen en effecten. Bedankt voor jullie hulp bij het schrijven van artikelen, de coördinatie van de 
prakijken, het opstellen van vragenlijsten, het meekijken bij revisies en ga zo maar door! Het was heel fijn om de verbinding met gezondheidsbevordering via jullie te behouden.

Liesbeth Preller, Femke van Brussel, Dirk Schaars, Marloes Aalbers, Loek Dresen, PeterJan Mol, Elize van Ballegooie van het Kenniscentrum Sport (voormalig NISB) wil ik bedanken voor de samenwerking in dit project.

De bijdrage van BeweegKuurzorgverleners en -deelnemers was essentieel voor het gehele BeweegKuuronderzoek. Ik wil de zorgverleners bedanken voor de inzet bij het includeren, het begeleiden en het meten van de deelnemers. In het bijzonder voor jullie doorzettingsvermogen en geduld met de activiteitenmonitors. Jullie motiveerden mij om iedere keer weer de trein in te stappen voor een vele uren durende reis door Nederland. Daarom, bedankt aan alle praktijkverpleegkundigen, fysiotherapeuten, diëtisten, huisartsen en leefstijladviseurs in onze onderzoekslocaties: Wijckel, Ferwerd, Delfzijl, Ommen, Assen, Nieuw Amsterdam, Nieuw Schoonebeek, Schoonebeek, Zwolle, Nijverdal, Nijkerk, Maarssen, Ede, Nijmegen, Enschede, Eindhoven, Amsterdam, Sittard, Buchten, 's Gravenzande, Den Haag, Wassenaar, Rotterdam, Berkel en Rodenrijs, Etten-Leur, Zundert en Hulst. Ook de ROS-adviseurs bedankt voor de inzet om de BeweegKuur zo soepel mogelijk te laten verlopen. De deelnemers bedank ik, o.a. voor het invullen van maar liefst negen vragenlijsten. We ontvingen zelfs kerstkaartjes van jullie! lk hoop dat jullie door de BeweegKuur een stap hebben kunnen maken op weg naar een gezonde leefwijze.

Co-auteurs, Kenneth en Guy bedank ik voor hun hulp en uitleg toen ik met de accelerometers begon te werken. Ik heb het erg fijn gevonden dat ik bij jullie terecht kon. Paul, bedankt voor de vele uurtjes overleg en het programmeren van de software. De afdeling mag zijn handjes dichtknijpen met een collega als jij.

Collega's, bedankt voor het meermaals dragen van accelerometers voor mijn 'minionderzoekjes' (“dit is echt de laatste keer dat ze gedragen moeten worden!”). De sfeer in de labgroep was een goede reden om (bijna) iedere dag weer met plezier richting Maastricht te reizen. Ik zal de gezellige lunches, ALBAS, vrijdagmiddagborrels en labmeetings missen. Mr. T, bedankt voor de gezellige kletsmomenten en voor de nuttige tips als ik eens een moeizame onderwijsgroep onder mijn hoede had. Desirée, 
secretaresse en voormalige buurvrouw, bedankt voor de gezellige praatjes en de hulp bij alle praktische kantjes van promoveren.

En na 3,5 jaar bij BW kwam ik op kamer 2.220 terecht. Wat was dat een leuke tijd! Uitstapjes naar Eindhoven, avondje geocachen, ijsjes eten in Epen en algehele gezelligheid. Ik wil hierbij Michiel nog een keer bedanken voor de hulp met Matlab en je pogingen om mijn muzieksmaak (in jouw ogen) te verbeteren. Verder denk ik dat de hele kamer jou dank verschuldigd is voor alle taart die dankzij jou bijna elke maandag gegeten kon worden. Mijn overbuurman Pieter, de rust zelve en topgeheimenbewaarder toen ik net zwanger was van Lucas. Ik heb genoten van je droge opmerkingen en ik moet nog lachen als ik terugdenk hoe vaak ik tegen mezelf aan het praten bleek te zijn. En Hanzl, hoe grappig is het? Nu zeven jaar na onze gezamenlijke stage sta ik hier te promoveren en ben jij ook met je eigen onderzoek bezig. De chocomel-momentjes en Matlab-hulp waren onmisbaar. Last, but not least, Berend Brokkepap, de jongste telg van het clubje met de grootste ambities. Het was altijd leuk om met jou over sedentair gedrag te discussiëren (serieus en soms wat minder serieus), ik ben erg blij dat onze interesses (wat betreft onderzoek) op elkaar aansluiten.

Mijn maatje in Zwitserland, Ursina, wat ben ik blij dat we elkaar nog goed hebben leren kennen aan het eind van je jaartje in Nederland. Af en toe een reisje Zurich-Limburg en omgekeerd is altijd de moeite waard gebleken. Hopelijk volgen er nog meer samen met Jan en Lucas!

Voor gesprekken over onderzoek en carrière, of gewoon voor gezelligheid, kon ik altijd terecht bij Tom. Je kritische blik heeft mij geholpen bij de interpretatie van de effecten van de BeweegKuur en bij het afronden van het proefschrift. Al reageerde ik niet altijd even enthousiast op je 'noordelijke' manier van feedback geven, ik ben blij dat je zo goed hebt meegekeken bij de laatste puntjes op de i. Ik heb ook heel wat hersentraining te danken aan je (in ieder geval voor mij) soms moeilijke humor.

Karianna, de trein is wat mij betreft de perfecte plek om even af te schakelen, of om juist verder te kletsen over multilevel analyses en plotjes. De lunches met jou, Eveline en Dorit ga ik natuurlijk niet vergeten! We hebben ongeveer alle onderwerpen wel gehad tijdens de boterhammetjes, maar de aankondigingen van ingediende manuscripten, geaccepteerde artikelen en natuurlijk baby's waren toch de hoogtepunten! Het was erg fijn om bij jullie 
mijn ei kwijt te kunnen als er iets niet helemaal ging zoals gepland/gehoopt/verwacht. Nog leuker is het dat we het samen eten niet verleerd hebben, ook al werken we nu allemaal ergens anders.

Denise, we hebben al veel samen gedaan, waarbij privé en werk altijd overlapt. Dat wij nu collega's zijn, bewijst maar weer hoe onze interesses op elkaar aansluiten en hoe leuk het is om samen te werken. Wie had dat gedacht 16 jaar geleden? Wij niet in ieder geval! En natuurlijk zijn we niet compleet zonder Britt en Denise. Wat ben ik blij vriendinnen te hebben waarbij ik altijd terecht kan en dat we in die jaren al zoveel hebben gedeeld.

Mijn geweldige thuis was en is, als huismus, de perfecte basis om fijn te kunnen studeren en werken. Vooral de keukentafel met de felle spotjes was een heerlijke werkplek (paps, de lichtrekening maak ik nog wel eens goed). We spreken het meestal niet zo uit, maar ik ben erg dankbaar voor de liefde en steun van jullie allemaal. Jullie zijn de beste, leukste en meest gezellige familie die ik me kan wensen - paps, mams, Lotte, Lars, Damiano en Bea.

Tot slot, mijn zelfgemaakte thuis, met Roy en Lucas is de allermooiste reden om me voor in te zetten. Roy, ik wil je hierbij nog eens laten weten hoe je hebt bijgedragen aan dit boekje door geduldig mijn verhalen en frustraties aan te horen, conceptversies te lezen, posters te controleren, etcetera, maar vooral door je vertrouwen in mij en mijn werk. En Lucas, jij bent gewoon de liefste. 

About the author 



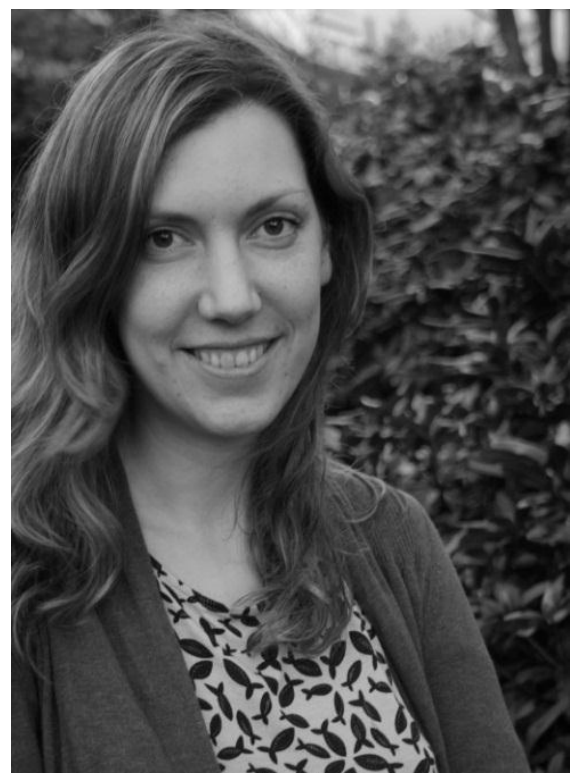

Brenda Berendsen was born in Heerlen, January 13th in 1987. When she was nine months old she and her family moved to Beek, where she grew up. She attended secondary school at Graaf Huyn College in Geleen and graduated in 2005. From 2005 till 2008 she studied General Health Sciences at Maastricht University, with a specialisation in Health Education and Human Movement Sciences. During her bachelor, she completed the Health Sciences honours programme. She obtained her master's degree in Human Movement Sciences in 2009 as a top 3\% student.

After graduation Brenda worked as a research assistant at the departments of Movement Sciences and Economics at Maastricht University. In April 2010, she started her PhD trajectory at Movement Sciences, aimed at the (cost-)effectiveness of the BeweegKuur combined lifestyle intervention. Besides the BeweegKuur project, she developed her expertise in the measurement of physical behaviour by means of accelerometers. Her research has led to several scientific publications in international peer-reviewed journals and presentations at both national and international conferences.

Brenda currently lives in Elsloo with her family and works as a teacher and project leader at the Open University in Heerlen at the department of Psychology and Educational Sciences. Her project is aimed at the adaptation, implementation and evaluation of an intervention to promote physical activity in older adults. 



\section{List of publications}





\section{Publications}

Berendsen BAJ, Hendriks MRC, Verhagen EALM, Schaper NC, Kremers SPJ, Savelberg HHCM. Effectiveness and cost-effectiveness of BeweegKuur, a combined lifestyle intervention in the Netherlands: Rationale, design and methods of a randomized controlled trial. BMC Public Health 20I I, I I:8I 5.

Berendsen BAl, Hendriks MRC, Meijer K, Plasqui G, Schaper NC, Savelberg HHCM. Which activity monitor to use? Validity, reproducibility and user friendliness of three activity monitors. BMC Public Health 20 I4, I4:749 (highly accessed).

Berendsen BAJ, Hendriks MRC, Willems P, Meijer K, Schaper NC, Savelberg HHCM. A 20 min window is optimal in a nonwear algorithm for tri-axial thigh-worn accelerometry in overweight people. Physiological Measurement 2014, 35:2205-22I 2.

Berendsen BAJ, Kremers SPJ, Schaper NC, Savelberg HHCM, Hendriks MRC. The implementation and sustainability of a combined lifestyle intervention in primary care: mixed method process evaluation. BMC Family Practice 2015, 16:37 (highly accessed).

De Vries S, Langers F, Meis JJM, Berendsen BAJ, Kremers SPJ. Blijven Bewegen na de BeweegKuur; De rol van groen in de woonomgeving. Alterra-rapport 27012016 Alterra Wageningen UR.

Berendsen BAJ, Hendriks MRC, Rutten GM, Kremers SPJ, Savelberg HHCM, Schaper NC. Effectiveness of the BeweegKuur, a combined lifestyle intervention for overweight people in primary care. Under review.

Meis JJM, Kremers SPJ, Rutten GM, Berendsen BAJ, Teixeira PJ, Savelberg HHCM, De Vries NK, Schols AMWJ. Quantity versus quality: Overweight adults' motivation regarding physical activity and healthy eating. Submitted.

Hendriks MRC, Berendsen BAJ, Verhagen EALM, Kremers SPJ, Schaper NC, Savelberg HHCM, Evers SMAA. Cost effectiveness and cost utility analyses of two intensities of a combined lifestyle intervention in primary care. Submitted. 


\section{Presentations}

Berendsen BAJ, Hendriks MRC, Kremers SPJ, Schaper NC, Savelberg HHCM. (20I5, June) Amount of guidance in a combined lifestyle intervention to improve lifestyle of overweight people: the more, the better? Poster presented at the Annual meeting of the International Society of Behavioural Nutrition and Physical Activity, Glasgow, UK.

Hendriks MRC, Berendsen BAJ, Verhagen EALM, Kremers SPJ, Savelberg HHCM. (20I5, June) Long-term cost-effectiveness of a combined lifestyle intervention aimed at improving quality of life from a societal perspective. Presented at the Annual meeting of the International Society of Behavioural Nutrition and Physical Activity, Glasgow, UK.

Berendsen BAJ, Hendriks MRC, Kremers SPJ, Schaper NC, Savelberg HHCM. (20I5, April) Effectiviteit van de BeweegKuur: Is meer begeleiding beter? Symposium presentation at NCVGZ, Rotterdam, NL.

Hendriks MRC, Berendsen BAJ, Verhagen EALM, Kremers SPJ, Savelberg HHCM. (20I5, April) Lange termijn kosteneffectiviteit van het Opstart beweegprogramma versus Begeleid beweegprogramma van de BeweegKuur. Symposium presentation at NCVGZ, Rotterdam, NL.

Hendriks MRC, Berendsen BAJ, Verhagen EALM, Kremers SPJ, Savelberg HHCM. (20I4, June). Cost-effectiveness of two intensities of a combined lifestyle intervention. Presented at the ECSS, Amsterdam, NL.

Berendsen BAJ, Hendriks MRC, Savelberg HHCM, Schaper NC, Kremers SPJ. (20I4, June) Process evaluation of a combined lifestyle intervention in Dutch primary care. Presented at the Netherlands Society for Movement Sciences PhD day, Groningen, NL.

Berendsen BAJ, Hendriks MRC, Savelberg HHCM, Schaper NC, Kremers SPJ. (20I4, May) Process evaluation of a combined lifestyle intervention in Dutch primary care. Presented at the Annual meeting of the International Society of Behavioural Nutrition and Physical Activity, San Diego, US.

Hendriks MRC, Berendsen BAJ, Kremers SPJ, Savelberg HHCM. (2014, May). Costeffectiveness of two intensities of a combined lifestyle intervention aimed at improving physical activity and nutritional behavior. Poster presented at the Annual meeting of the International Society of Behavioural Nutrition and Physical Activity, San Diego, US.

Rutten GM, Berendsen BAJ, Meis JJM, Kremers SPJ. (2014, May). Does healthcare professionals' performance in a combined lifestyle intervention for overweight and obese individuals affect changes in motivation and behaviour? Poster presented at the Annual 
meeting of the International Society of Behavioural Nutrition and Physical Activity, San Diego, US.

Berendsen BAJ, Hendriks MRC, Savelberg HHCM, Schaper NC, Kremers SPJ. (20I4, April) Evaluatie van de uitvoering van een gecombineerde leefstijlinterventie in de eerste lijn. Symposium presentation at NCVGZ, Rotterdam, NL.

Hendriks MRC, Berendsen BAJ, Verhagen EALM, Kremers SPJ, Savelberg HHCM. (20I4, April) Kosteneffectiviteit van het Opstart beweegprogramma versus Begeleid beweegprogramma van de BeweegKuur. Presented at NCVGZ, Rotterdam, NL.

Rutten GM, Berendsen BAJ, Meis JJM, Kremers SPJ. (2014, April) Leidt leefstijlcoaching tot meer autonome motivatie en gezonder gedrag bij deelnemers aan een gecombineerde leefstijlinterventie? Symposium presentation at NCVGZ, Rotterdam, NL.

Berendsen BAJ, Hendriks MRC, Savelberg HHCM, Schaper NC, Kremers SPJ. (20I4, April) Process evaluation of a combined lifestyle intervention in Dutch primary care. Poster presented at the meeting of Nederlandse Associatie voor de Studie van Obesitas, Oosterbeek, NL.

Berendsen BAJ, Hendriks MRC, Meis JJM, Schaper NC, Savelberg HHCM. (20I3, May) Sitting time, physical activity and body composition in a population of overweight and obese adults. Poster presented at the Annual meeting of the International Society of Behavioural Nutrition and Physical Activity, Ghent, BE.

Berendsen BAJ, Hendriks MRC, Schaper NC, Willems P, Meijer K, Savelberg HHCM. (2013, June) Non-wear algorithm accuracy in an overweight population. Poster presented at the International Conference on Ambulatory Monitoring of Physical Activity and Movement, Amherst, US.

Berendsen BAJ, Hendriks MRC, Verhagen EALM, Schaper NC, Kremers SPJ, Savelberg HHCM. (20II, October). The effectiveness of BeweegKuur, a combined lifestyle intervention in the Netherlands: rationale and design of a controlled trial. Poster presented at the Health Enhancing Physical Activity Conference, Amsterdam, NL.

Berendsen BAJ, Annegarn J, Hendriks MRC, Schaper NC, Willems P, Plasqui G, Meijer K, Savelberg HHCM. (201I, May) Validity and user friendliness of three activity monitors. Presented at the International Conference on Ambulatory Monitoring of Physical Activity and Movement, Glasgow, UK. 